Supporting information.

\title{
Efficient Synthesis of the Indoloazocine Framework via Intramolecular Alkyne Carbocyclization.
}

Pavel A. Donets ${ }^{\mathrm{a}}$, Kristof Van Hecke ${ }^{\mathrm{b}}$, Luc Van Meervelt ${ }^{\mathrm{b}}$ and Erik V. Van der Eycken ${ }^{\mathrm{a} *}$

a Laboratory for Organic \& Microwave-Assisted Chemistry (LOMAC), Department of Chemistry, Katholieke Universiteit Leuven, Celestijnenlaan 200F, B-3001 Leuven, Belgium

${ }^{\mathrm{b}}$ Biomolecular Architecture, Department of Chemistry, Katholieke Universiteit Leuven, Celestijnenlaan 200F, B-3001 Leuven, Belgium

* To whom correspondence should be addressed. E-mail: erik.vandereycken@chem.kuleuven.be

\section{Contents}

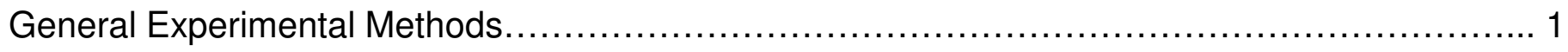

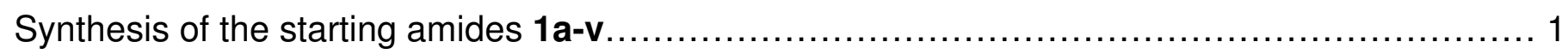

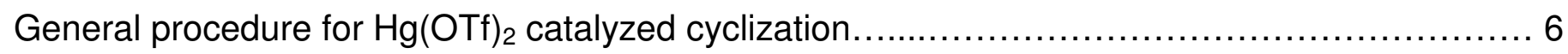

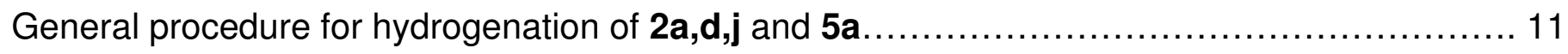

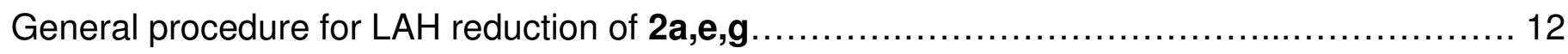

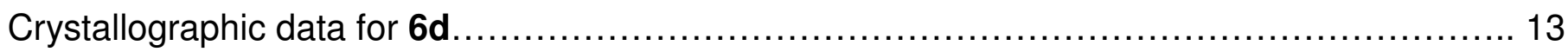

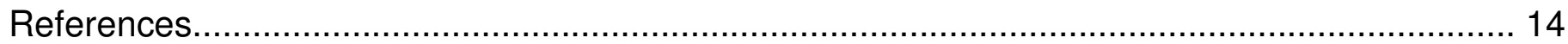

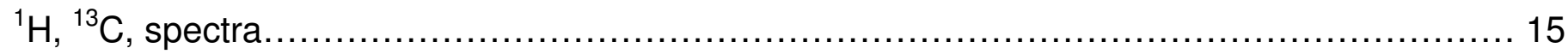




\section{General Experimental Methods.}

${ }^{1} \mathrm{H}$ and ${ }^{13} \mathrm{C}$ NMR spectra were recorded on Bruker Avance 300 or Bruker 400 instruments at $298 \mathrm{~K}$ unless otherwise stated. The ${ }^{1} \mathrm{H}$ and ${ }^{13} \mathrm{C}$ chemical shifts are reported in parts per million relative to tetramethylsilane using the residual solvent signal as the internal reference. Mass spectra were recorded on Kratos MS50TC and Kratos Mach III system. The ion source temperature was $150-250^{\circ} \mathrm{C}$, as required. High-resolution El-mass spectra were recorded with a resolution of 10000. The low-resolution spectra were obtained with HP5989A MS instrument. For thinlayer chromatography, analytical TLC plates (Alugram SIL G/UV254 and 70-230 mesh silica gel (E. M. Merck) were used. Optical rotations were measured using a PROPOL $®$ Automatic Process Polarimeter.

\section{Microwave Irradiation Experiments.}

A monomode CEM Discover ${ }^{\mathrm{TM}}$ microwave reactor was used in the standard configuration as delivered, including proprietary software. All experiments were carried out in sealed microwave process vials.

\section{Synthesis of the starting amides 1a-v:}

Amides 1a-v appear as a mixture of two rotamers (with the exception of secondary amide $1 \mathbf{d}$ ). ${ }^{13} \mathrm{C}$ spectra of 1a-v were not registered, since overlapping of multiple peaks of both rotamers (example 1a) makes the spectra not very characteristic. ${ }^{1} \mathrm{H}$ NMR spectra of $\mathbf{1 a - v}$ are described for both rotamers considering the ratio. $[\alpha]_{D}{ }^{20}$ values for enantiomerically pure $\mathbf{1 p}-\mathbf{v}$ were not measured. On TLC amides $\mathbf{1 a - v}$ often appear as two interconverting spots. The starting tryptamines 8-10 were prepared according to the references 1-3 respectively.

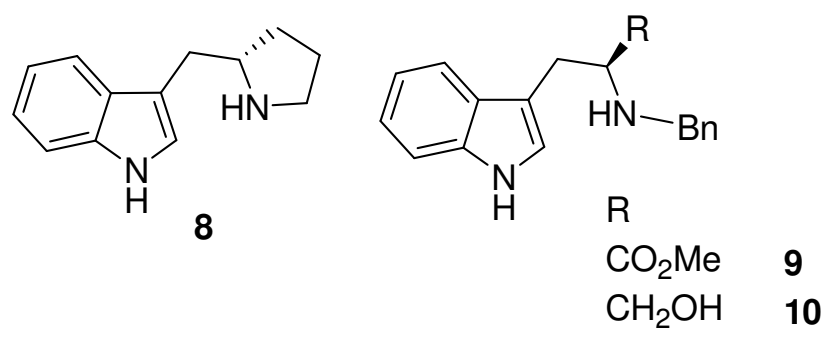

\section{General procedure for the synthesis of propynoic acid amides 1a-k,p-t,12a,b:}

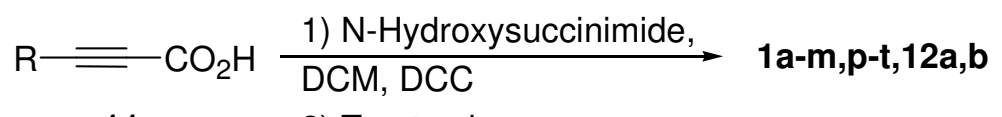

11
2) Tryptamine

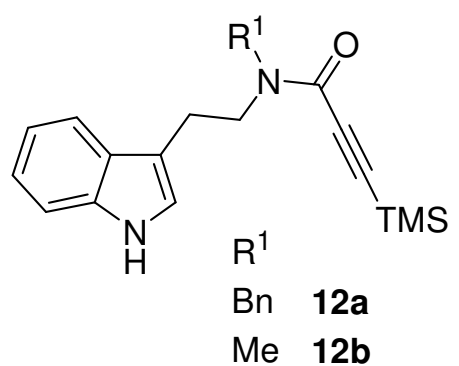

3 substituted 2-propynoic acid 11 (1.15 equiv) and N-hydroxysuccinimide $276 \mathrm{mg}$ (1.2 equiv) were dissolved in dry DCM (6 mL). DCC $475 \mathrm{mg}$ (1.15 equiv) was added and the reaction was stirred at ambient temperature for 3-4 $\mathrm{h}$. Tryptamine ( $2 \mathrm{mmol}, 1$ equiv) was added in one portion as solid or as a solution in DCM (4 mL) and the reaction was left with stirring overnight. The subsequently formed precipitate of $\mathrm{N}, \mathrm{N}$-dicyclohexylurea was filtered off and washed 
well with $\mathrm{Et}_{2} \mathrm{O}$. The organic layer was washed with successively with $\mathrm{HCl}(1 \mathrm{M}), \mathrm{NaHCO}_{3}$ (sat), brine, dried $\left(\mathrm{MgSO}_{4}\right)$ and concentrated with silica. Pure amides 1a-m,p-t were isolated by column chromatography on silica gel.

2-Butynoic acid benzyl-[2-(1 H-indol-3-yl)-ethyl]-amide (1a). Synthesized from tetrolic acid $194 \mathrm{mg}$ and 500 $\mathrm{mg}$ of $\mathrm{N}$-benzyltryptamine. Eluent DCM / $\mathrm{Et}_{2} \mathrm{O}(4 / 1)\left(R_{f} 0.41\right)$. Yield: $569 \mathrm{mg}(93 \%)$. (mixture of rotamers $\left.\sim 3 / 2\right){ }^{1} \mathrm{H}$ NMR (DMSO- $\left.d_{6}, 300 \mathrm{MHz}\right): \delta 10.84(\mathrm{bs}, 1 \mathrm{H}), 7.18-7.56(\mathrm{~m}, 7 \mathrm{H}), 6.91-7.15(\mathrm{~m}, 3 \mathrm{H}), 4.74(\mathrm{~s}, 0.8 \mathrm{H}), 4.59(\mathrm{~s}, 1.2 \mathrm{H})$, 3.64-3.74 (m, 1.2H), 3.41-3.49 (m, 0.8H), 2.91-2.98 (m, 1.2H), 2.78-2.86 (m, 0.8H), $2.02(\mathrm{~s}, 1.2 \mathrm{H}), 1.86(\mathrm{~s}, 1.8 \mathrm{H}) ;{ }^{13} \mathrm{C}$ NMR $\left(\mathrm{CDCl}_{3}, 100 \mathrm{MHz}\right): \delta 155.40,155.06,136.87,136.68,136.56,136.43,128.82,128.73,128.19,127.90,127.67$, $127.62,127.41,127.34,122.68,122.41,121.94,121.87,119.21,118.66,118.36,112.48,111.85,111.59,111.45$, 89.34, 89.31, 73.79, 73.35, 53.36, 48.84, 47.67, 45.21, 24.73, 23.11, 4.09, 3.73; LRMS(EI) (m/z, relative intensity) : $316\left(\mathrm{M}^{+} \cdot, 13\right), 143(100), 130(32), 91(22), 67(16)$

2-Hexynoic acid benzyl-[2-(1 H-indol-3-yl)-ethyl]-amide (1b). Synthesized from hex-2-ynoic acid acid $258 \mathrm{mg}$ and $500 \mathrm{mg}$ of $\mathrm{N}$-benzyltryptamine. Eluent DCM / $\mathrm{Et}_{2} \mathrm{O}(20 / 1)\left(R_{f} 0.27\right)$. Yield: $601 \mathrm{mg}(87 \%)$. (mixture of rotamers 3 / 2) ${ }^{1} \mathrm{H}$ NMR (DMSO- $\left.d_{6}, 400 \mathrm{MHz}\right): \delta ; 10.85$ (bs, $1 \mathrm{H}$ ), 7.50 (d, 0.6H, J = 7.5 Hz), 7.45 (d, 0.4H, J = 7.7 Hz), 7.24$7.41(\mathrm{~m}, 6 \mathrm{H})$, 7.03-7.12 (m, 2H), 6.94-7.00 (m, 1H), $4.76(\mathrm{~s}, 0.8 \mathrm{H}), 4.60(\mathrm{~s}, 1.2 \mathrm{H}), 3.66-3.74(\mathrm{~m}, 1.2 \mathrm{H}), 3.43-3.50(\mathrm{~m}$, $0.8 \mathrm{H}), 2.91-2.99(\mathrm{~m}, 1.2 \mathrm{H}), 2.80-2.86(\mathrm{~m}, 0.8 \mathrm{H}), 2.37(\mathrm{t}, 0.8 \mathrm{H}, J=6.9 \mathrm{~Hz}), 2.22(\mathrm{t}, 1.2 \mathrm{H}, 6.9 \mathrm{~Hz}), 1.39-1.53(\mathrm{~m}, 2 \mathrm{H})$, $0.90(\mathrm{t}, 3 \mathrm{H}, J=7.3 \mathrm{~Hz})$; LRMS(Cl) $\left(\mathrm{m} / \mathrm{z}\right.$, relative intensity) : $345\left([\mathrm{M}+\mathrm{H}]^{+}, 100\right), 277(6), 143(28)$

2-Butynoic acid [2-(1H-indol-3-yl)-ethyl]-(4-methoxybenzyl)-amide (1c). Synthesized from tetrolic acid 194 $\mathrm{mg}$ and $561 \mathrm{mg}$ of $\mathrm{N}$-(p-methoxybenzyl)tryptamine. Eluent DCM / $\mathrm{Et}_{2} \mathrm{O}(4 / 1)\left(R_{f} 0.35\right)$. Yield: $637 \mathrm{mg}(92 \%)$. (mixture of rotamers $\sim 3 / 2$ ) ${ }^{1} \mathrm{H}$ NMR (DMSO- $d_{6}, 300 \mathrm{MHz}$ ) : $\delta 10.84$ (bs, $1 \mathrm{H}$ ), $7.51(\mathrm{~d}, 0.6 \mathrm{H}, J=7.8 \mathrm{~Hz}), 7.45(\mathrm{~d}, 0.4 \mathrm{H}, J=7.7$ $\mathrm{Hz}), 7.33(\mathrm{~m}, 1 \mathrm{H}), 7.17-7.23(\mathrm{~m}, 2 \mathrm{H}), 7.03-7.11(\mathrm{~m}, 3 \mathrm{H}), 4.67(\mathrm{~s}, 0.8 \mathrm{H}), 4.52(\mathrm{~s}, 1.2 \mathrm{H}), 3.72-3.76(\mathrm{~m}, 3 \mathrm{H}), 3.62-3.69$ $(\mathrm{m}, 1.2 \mathrm{H}), 3.39-3.45(\mathrm{~m}, 0.8 \mathrm{H}), 2.89-2.95(\mathrm{~m}, 0.8 \mathrm{H}), 2.77-2.85(\mathrm{~m}, 1.2 \mathrm{H}), 2.03(\mathrm{~s}, 1.2 \mathrm{H}), 1.85(\mathrm{~s}, 1.8 \mathrm{H})$

2-Butynoic acid [2-(1H-indol-3-yl)-ethyl]-amide (1d). Synthesized from tetrolic acid $194 \mathrm{mg}$ and $320 \mathrm{mg}$ of tryptamine. Eluent DCM / EtOAc (1/1) $\left(R_{f} 0.45\right)$. Yield: $376 \mathrm{mg}(83 \%) .{ }^{1} \mathbf{H}$ NMR (DMSO- $\left.d_{6}, 300 \mathrm{MHz}\right): \delta 10.81$ (bs, $1 \mathrm{H}), 8.58(\mathrm{~m}, 1 \mathrm{H}), 7.52(\mathrm{~d}, 1 \mathrm{H}, J=7.6 \mathrm{~Hz}), 7.35(\mathrm{~d}, 1 \mathrm{H}, J=7.6 \mathrm{~Hz}), 7.15(\mathrm{~d}, 1 \mathrm{H}, J=1.7 \mathrm{~Hz}), 7.07(\mathrm{t}, 1 \mathrm{H}, J=7.6 \mathrm{~Hz})$, $6.99\left(\mathrm{t}, 1 \mathrm{H}, J=7.6 \mathrm{~Hz}\right.$ ), 3.32-3.38 (m, 2H), 2.80-2.88 (m, 2H), $1.95(\mathrm{~s}, 3 \mathrm{H}) ;{ }^{13} \mathrm{C}$ NMR (DMSO-d, $\left.75 \mathrm{MHz}\right): \delta 152.97$, 136.69, 127.63, 123.10, 121.40, 118.72, 118.66, 111.98, 111.83, 82.56, 76.29, 40.15, 25.25, 3.46.

2-Butynoic acid [2-(1H-indol-3-yl)-ethyl]-methylamide (1e). Synthesized from tetrolic acid $194 \mathrm{mg}$ and 348 $\mathrm{mg}$ of $\mathrm{N}$-methyltryptamine. Eluent DCM / $\mathrm{Et}_{2} \mathrm{O}(4 / 1)\left(R_{f} 0.29\right.$ and $\left.R_{f} 0.18\right)$. Yield: $425 \mathrm{mg}(88 \%)$. (mixture of rotamers $\sim 3 / 2)^{1} \mathrm{H}$ NMR (DMSO- $\left.d_{6}, 300 \mathrm{MHz}\right): \delta 10.84(\mathrm{bs}, 1 \mathrm{H}), 7.53-7.61(\mathrm{~m}, 1 \mathrm{H}), 7.30-7.39(\mathrm{~m}, 1 \mathrm{H}), 7.11-7.17(\mathrm{~m}, 1 \mathrm{H})$, 6.95-7.10 (m, 2H), 3.71-3.81 (m, 1.2H), 3.52-3.61 (m, 0.8H), $3.10(\mathrm{~s}, 1.2 \mathrm{H}), 2.83-2.99(\mathrm{~m}, 3.8 \mathrm{H}), 2.01(\mathrm{~s}, 1.2 \mathrm{H}), 1.79$ (s, 1.8H); LRMS(Cl) (m/z, relative intensity) : $241\left([\mathrm{M}+\mathrm{H}]^{+}, 100\right), 201$ (6), $172(8), 144$ (42), 143 (32).

2-Pentynoic acid [2-(1H-indol-3-yl)-ethyl]-methylamide (1f). Synthesized from 2-pentynoic acid $225 \mathrm{mg}$ and $348 \mathrm{mg}$ of $\mathrm{N}$-methyltryptamine. Eluent DCM / $\mathrm{Et}_{2} \mathrm{O}(4 / 1)\left(R_{f} 0.23\right.$ and $\left.R_{f} 0.32\right)$. Yield: $451 \mathrm{mg}(89 \%)$. (mixture of rotamers 13 / 7) ${ }^{1} \mathrm{H}$ NMR (DMSO- $\left.d_{6}, 300 \mathrm{MHz}\right): \delta 10.83$ (bs, $1 \mathrm{H}$ ), $7.56(\mathrm{~d}, 1 \mathrm{H}, J=7.8 \mathrm{~Hz}$ ), $7.34(\mathrm{~d}, 1 \mathrm{H}, J=7.8 \mathrm{~Hz}$ ), 6.94-7.17 (m, 3H), $3.76(t, 1.3 \mathrm{H}, J=7.1 \mathrm{~Hz}), 3.57(\mathrm{t}, 0.7 \mathrm{H}, J=7.7 \mathrm{~Hz}), 3.11(\mathrm{~s}, 1.05 \mathrm{H}), 2.95(\mathrm{t}, 1.3 \mathrm{H}, J=7.1 \mathrm{~Hz})$, 
2.80-2.90 (m, 2.65H), 2.39 (q, 0.7H, $J=7.4 \mathrm{~Hz}), 2.17(\mathrm{q}, 1.3 \mathrm{H}, J=7.4 \mathrm{~Hz}), 1.12(\mathrm{t}, 1.05 \mathrm{H}, J=7.4 \mathrm{~Hz}), 0.99$ (t, $1.95 \mathrm{H}$, $J=7.4 \mathrm{~Hz}$ ); LRMS(Cl) (m/z, relative intensity) : 255 ([M+H] $\left.]^{+}, 100\right), 225$ (11), 201 (5), 172 (5), 144 (17), 143 (15).

2-Hexynoic acid [2-(1 H-indol-3-yl)-ethyl]-methylamide (1 $\mathrm{g})$. Synthesized from 2-hexynoic $258 \mathrm{mg}$ and 348 $\mathrm{mg}$ of $\mathrm{N}$-methyltryptamine. Eluent DCM / $\mathrm{Et}_{2} \mathrm{O}(4 / 1)\left(R_{f} 0.28\right.$ and $\left.R_{f} 0.38\right)$. Yield: $529 \mathrm{mg}(99 \%)$. (mixture of rotamers $\sim 13 / 7)^{1} \mathrm{H}$ NMR (DMSO- $d_{6}, 300 \mathrm{MHz}$ ) : $\delta 10.84$ (bs, $1 \mathrm{H}$ ), $7.56(\mathrm{~d}, 1 \mathrm{H}, J=7.8 \mathrm{~Hz}$ ), $7.34(\mathrm{~d}, 1 \mathrm{H}, J=7.8 \mathrm{~Hz}$ ), 6.94-7.17 $(\mathrm{m}, 3 \mathrm{H}), 3.76(\mathrm{t}, 1.3 \mathrm{H}, J=7.4 \mathrm{~Hz}), 3.57(\mathrm{t}, 0.7 \mathrm{H}, J=7.4 \mathrm{~Hz}), 3.11(\mathrm{~s}, 1.05 \mathrm{H}), 2.80-2.99(\mathrm{~m}, 3.95 \mathrm{H}), 2.37(\mathrm{t}, 0.7 \mathrm{H}, J=$ $7.4 \mathrm{~Hz}), 2.15(\mathrm{t}, 1.3 \mathrm{H}, J=7.4 \mathrm{~Hz}), 1.46-1.60(\mathrm{~m}, 0.7 \mathrm{H}), 1.32-1.45(\mathrm{~m}, 1.3 \mathrm{H}), 0.96(\mathrm{t}, 1.05 \mathrm{H}, J=7.4 \mathrm{~Hz}), 0.86(\mathrm{t}, 1.95 \mathrm{H}$, $J=7.4 \mathrm{~Hz}$ ); LRMS(Cl) (m/z, relative intensity) : 269 ([M+H $\left.]^{+}, 100\right), 201$ (7), 172 (6), 144 (21), 143 (25).

4-Methyl-2-pentynoic acid [2-(1H-indol-3-yl)-ethyl]-methylamide (1h). Synthesized from 4-methyl-2pentynoic acid $258 \mathrm{mg}$ and $348 \mathrm{mg}$ of $\mathrm{N}$-methyltryptamine. Eluent DCM / $\mathrm{Et}_{2} \mathrm{O}(4 / 1)\left(R_{f} 0.33\right.$ and $\left.R_{f} 0.38\right)$. Yield: 476 $\mathrm{mg}(89 \%)$. (mixture of rotamers 13/7) ${ }^{1} \mathrm{H}$ NMR (DMSO- $\left.d_{6}, 300 \mathrm{MHz}\right): \delta 10.84(\mathrm{bs}, 1 \mathrm{H}), 7.52-7.61(\mathrm{~m}, 1 \mathrm{H}), 7.30-$ $7.38(\mathrm{~m}, 1 \mathrm{H}), 6.94-7.17(\mathrm{~m}, 3 \mathrm{H}), 3.75(\mathrm{t}, 1.3 \mathrm{H}, J=7.4 \mathrm{~Hz}), 3.56(\mathrm{t}, 0.7 \mathrm{H}, J=7.4 \mathrm{~Hz}), 3.11(\mathrm{~s}, 1.05 \mathrm{H}), 2.73-2.99(\mathrm{~m}$, 4.3H), 2.56 (septet, $0.65 \mathrm{H}, J=6.7 \mathrm{~Hz}), 1.17(\mathrm{~d}, 2.1 \mathrm{H}, J=6.7 \mathrm{~Hz}), 1.04(\mathrm{~d}, 3.9 \mathrm{H}, J=6.7 \mathrm{~Hz}) ; \mathbf{L R M S}(\mathrm{Cl})(\mathrm{m} / \mathrm{z}$, relative intensity) : $269\left([\mathrm{M}+\mathrm{H}]^{+}, 100\right), 225$ (3), 201 (5), 172 (6), 144 (20), 143 (23), 95 (9).

4,4-Dimethyl-2-pentynoic acid [2-(1H-indol-3-yl)-ethyl]-methylamide (1i). Synthesized from 4,4-dimethyl-2pentynoic acid $290 \mathrm{mg}$ and $348 \mathrm{mg}$ of $\mathrm{N}$-methyltryptamine. Eluent DCM / $\mathrm{Et}_{2} \mathrm{O}(4 / 1)\left(R_{f} 0.34\right.$ and $\left.R_{f} 0.41\right)$. Yield: 485 $\mathrm{mg}(86 \%)$. (mixture of rotamers $~ 13 / 7){ }^{1} \mathrm{H}$ NMR (DMSO- $\left.d_{6}, 300 \mathrm{MHz}\right): \delta 10.85(\mathrm{bs}, 1 \mathrm{H}), 7.52-7.61(\mathrm{~m}, 1 \mathrm{H}), 7.30-$ $7.39(\mathrm{~m}, 1 \mathrm{H}), 6.93-7.19(\mathrm{~m}, 3 \mathrm{H}), 3.75(\mathrm{t}, 1.3 \mathrm{H}, J=7.4 \mathrm{~Hz}), 3.56(\mathrm{t}, 0.7 \mathrm{H}, J=7.4 \mathrm{~Hz}), 3.11(\mathrm{~s}, 1.05 \mathrm{H}), 2.95(\mathrm{t}, 1.3 \mathrm{H}, J=$ $7.4 \mathrm{~Hz}), 2.81-2.90(\mathrm{~m}, 2.65 \mathrm{H}), 1.25(\mathrm{~s}, 3.15 \mathrm{H}), 1.11(\mathrm{~s}, 5.85)$; LRMS(Cl) $\left(\mathrm{m} / \mathrm{z}\right.$, relative intensity) : $283\left([\mathrm{M}+\mathrm{H}]^{+}, 100\right)$, $201(4), 172(6), 144(16), 143(29)$.

3-Phenylpropynoic acid [2-(1 H-indol-3-yl)-ethyl]-methylamide (1j). Synthesized from 3-phenylpropynoic acid $336 \mathrm{mg}$ and $348 \mathrm{mg}$ of $\mathrm{N}$-methyltryptamine. Eluent $\mathrm{DCM} / \mathrm{Et}_{2} \mathrm{O}(4 / 1)\left(R_{f} 0.33\right.$ and $\left.R_{f} 0.44\right)$. Yield: $553 \mathrm{mg}(91 \%)$. (mixture of rotamers 13/7) ${ }^{1} \mathrm{H}$ NMR (DMSO- $\left.d_{6}, 300 \mathrm{MHz}\right): \delta 3.85(\mathrm{~s}, 1 \mathrm{H}), 7.28-7.66(\mathrm{~m}, 7 \mathrm{H}), 7.13-7.21(\mathrm{~m}, 1 \mathrm{H})$, 6.96-7.12 (m, 1.35H), $6.83(\mathrm{t}, 0.65 \mathrm{H}, J=7.4 \mathrm{~Hz}), 3.86(\mathrm{t}, 1.3 \mathrm{H}, J=7.4 \mathrm{~Hz}), 3.65(\mathrm{t}, 0.7 \mathrm{H}, J=7.4 \mathrm{~Hz}), 3.22(\mathrm{~s}, 1.05 \mathrm{H})$, 2.89-3.07 (m, 3.95H); LRMS(Cl) (m/z, relative intensity) : 303 ([M+H] $\left.]^{+}, 100\right), 201$ (3), $172(2), 144(9), 143$ (13).

3-(3,4,5-Trimethoxyphenyl)propynoic acid [2-(1H-indol-3-yl)-ethyl]-methylamide (1k). Synthesized from 3(3,4,5-trimethoxyphenyl)propynoic acid $543 \mathrm{mg}$ and $348 \mathrm{mg}$ of $\mathrm{N}$-methyltryptamine. Eluent DCM / $\mathrm{Et}_{2} \mathrm{O}(4 / 1)\left(R_{f} 0.17\right.$ and $\left.R_{f} 0.24\right)$. Yield: $471 \mathrm{mg}(60 \%)$. (mixture of rotamers $\left.~ 13 / 7\right){ }^{1} \mathbf{H}$ NMR (DMSO- $\left.d_{6}, 400 \mathrm{MHz}\right): \delta 1.85$ (bs, $1 \mathrm{H}$ ), 7.54-7.61 (m, 1H), 7.29-7.37 (m, 1H), 7.16-7.22 (m, 1H), 6.96-7.11 (m, 1.3H), $6.94(\mathrm{~s}, 0.7 \mathrm{H}), 6.82(\mathrm{t}, 0.7 \mathrm{H}, J=7.5$ $\mathrm{Hz}), 6.74(\mathrm{~s}, 1.3 \mathrm{H}), 3.84-3.93(\mathrm{~m}, 1.3 \mathrm{H}), 3.81(\mathrm{~s}, 2.1 \mathrm{H}), 3.73(\mathrm{~s}, 3.9 \mathrm{H}), 3.62-3.71(\mathrm{~m}, 3.7 \mathrm{H}), 3.22(\mathrm{~s}, 1.05 \mathrm{H}), 2.89-3.07$ (m, 3.95H); LRMS(EI) (m/z, relative intensity) : $392\left(\mathrm{M}^{+*}, 100\right), 377$ (16), 219 (56), 143 (78), 130 (44).

3-Trimethylsilanylpropynoic acid benzyl-[2-(1H-indol-3-yl)-ethyl]-amide (12a). Synthesized from 3trimethylsilanylpropynoic acid $327 \mathrm{mg}$ and $500 \mathrm{mg}$ of $\mathrm{N}$-benzyltryptamine. Eluent DCM / $\mathrm{Et}_{2} \mathrm{O}(20 / 1)\left(R_{f} 0.44\right)$. Yield: $702 \mathrm{mg}(94 \%)$. (mixture of rotamers $3 / 2)^{1} \mathrm{H}$ NMR (DMSO- $\left.d_{6}, 400 \mathrm{MHz}\right): \delta 10.85(\mathrm{bs}, 0.6 \mathrm{H}), 10.80(\mathrm{bs}, 0.4 \mathrm{H}), 7.50$ $(\mathrm{d}, 0.6 \mathrm{H}, J=7.9 \mathrm{~Hz}), 7.46(\mathrm{~d}, 0.4 \mathrm{H}, J=7.9 \mathrm{~Hz}), 7.25-7.42(\mathrm{~m}, 6 \mathrm{H}), 7.03-7.13(\mathrm{~m}, 2 \mathrm{H}), 6.94-6.99(\mathrm{~m}, 1 \mathrm{H}), 4.76(\mathrm{~s}$, 
$0.8 \mathrm{H}), 4.63(\mathrm{~s}, 1.2 \mathrm{H}), 3.69-3.76(\mathrm{~m}, 1.2 \mathrm{H}), 3.47-3.53(\mathrm{~m}, 0.8 \mathrm{H}), 2.93-3.00(\mathrm{~m}, 1.2 \mathrm{H}), 2.81-2.86(\mathrm{~m}, 0.8 \mathrm{H}), 0.17-0.20$ $(\mathrm{m}, 9 \mathrm{H}) ;$ ); LRMS(Cl) $\left(\mathrm{m} / \mathrm{z}\right.$, relative intensity) : $375\left([\mathrm{M}+\mathrm{H}]^{+}, 100\right), 359(21), 143$ (32).

3-Trimethylsilanylpropynoic acid [2-(1H-indol-3-yl)-ethyl]-methylamide (12b). Synthesized from 3trimethylsilanylpropynoic acid $327 \mathrm{mg}$ and $348 \mathrm{mg}$ of $N$-methyltryptamine. Eluent DCM / $\mathrm{Et}_{2} \mathrm{O}(4 / 1)\left(R_{f} 0.36\right.$ and $R_{f}$ 0.48). Yield: $581 \mathrm{mg}(97 \%)$. (mixture of rotamers $3 / 2)^{1}{ }^{1} \mathrm{H}$ NMR (DMSO- $d_{6}, 400 \mathrm{MHz}$ ) : $\delta 10.88$ (bs, $\left.0.6 \mathrm{H}\right), 10.83$ (bs, 0.4H), $7.56(\mathrm{~d}, 1 \mathrm{H}, J=7.8 \mathrm{~Hz}), 7.32-7.37(\mathrm{~m}, 1 \mathrm{H}), 7.16(\mathrm{~d}, 0.4 \mathrm{H}, J=2.1 \mathrm{~Hz}), 7.13(\mathrm{~d}, 0.6 \mathrm{H}, J=2.1 \mathrm{~Hz}), 7.05-7.10$ $(\mathrm{m}, 1 \mathrm{H}), 6.95-7.01(\mathrm{~m}, 1 \mathrm{H}), 3.75-3.82(\mathrm{~m}, 1.2 \mathrm{H}), 3.55-3.61(\mathrm{~m}, 0.8 \mathrm{H}), 3.14(\mathrm{~s}, 1.2 \mathrm{H}), 2.94-3.00(\mathrm{~m}, 1.2 \mathrm{H}), 2.86-2.92$ $(\mathrm{m}, 2.6 \mathrm{H}), 0.23(\mathrm{~s}, 3.6 \mathrm{H}), 0.15(\mathrm{~s}, 5.4 \mathrm{H})$; LRMS(Cl) (m/z, relative intensity) : $299\left([\mathrm{M}+\mathrm{H}]^{+}, 100\right), 283(21), 172(6), 144$ (13), $143(21)$.

1-[(S)-2-(1 H-Indol-3-ylmethyl)-pyrrolidin-1-yl]-but-2-yn-1-one (1p). Synthesized from tetrolic acid $194 \mathrm{mg}$ and amine $8401 \mathrm{mg}$. Eluent DCM / $\mathrm{Et}_{2} \mathrm{O}(4 / 1)\left(R_{f} 0.15\right.$ and $\left.R_{f} 0.25\right)$. Yield: $383 \mathrm{mg}(72 \%)$. (mixture of rotamers $\left.\sim 1 / 1\right)$ ${ }^{1} \mathrm{H}$ NMR (DMSO- $\left.d_{6}, 300 \mathrm{MHz}\right): \delta 10.90(\mathrm{bs}, 0.5 \mathrm{H}), 10.84(\mathrm{bs}, 0.5 \mathrm{H}), 7.55-7.67(\mathrm{~m}, 1 \mathrm{H}), 7.29-7.39(\mathrm{~m}, 1 \mathrm{H}), 6.93-7.20$ $(\mathrm{m}, 3 \mathrm{H}), 4.25-4.37(\mathrm{~m}, 0.5 \mathrm{H}), 4.13-4.23(\mathrm{~m}, 0.5 \mathrm{H}), 3.45-3.61(\mathrm{~m}, 1 \mathrm{H}), 3.27-3.38(\mathrm{~m}, 1 \mathrm{H}), 3.02-3.17(\mathrm{~m}, 1 \mathrm{H}), 2.74-2.86$ $(\mathrm{m}, 0.5 \mathrm{H}), 2.58-2.70(\mathrm{~m}, 0.5 \mathrm{H}), 1.95-2.06(\mathrm{~m}, 3 \mathrm{H}), 1.59-1.92(\mathrm{~m}, 4 \mathrm{H})$; LRMS(Cl) (m/z, relative intensity) : $267\left([\mathrm{M}+\mathrm{H}]^{+}\right.$, 100), 227 (7), 136 (27).

1-[(S)-2-(1H-Indol-3-ylmethyl)-pyrrolidin-1-yl]-pent-2-yn-1-one (1q). Synthesized from 2-pentynoic acid 226 $\mathrm{mg}$ and amine $8401 \mathrm{mg}$. Eluent DCM / $\mathrm{Et}_{2} \mathrm{O}(4 / 1)\left(R_{f} 0.23\right.$ and $\left.R_{f} 0.34\right)$. Yield: $480 \mathrm{mg}(86 \%)$. (mixture of rotamers $1 / 1){ }^{1} \mathrm{H}$ NMR (DMSO- $\left.d_{6}, 400 \mathrm{MHz}\right): \delta 10.89$ (bs, 0.5H), 10.83 (bs, 0.5H), $7.64(\mathrm{~d}, 0.5 \mathrm{H}, J=7.8 \mathrm{~Hz}), 7.59(\mathrm{~d}, 0.5 \mathrm{H}, J$ $=7.8 \mathrm{~Hz}), 7.31-7.38(\mathrm{~m}, 1 \mathrm{H}), 7.16(\mathrm{~d}, 0.5 \mathrm{H}, J=1.9 \mathrm{~Hz}), 7.14(\mathrm{~d}, 0.5 \mathrm{H}, J=1.9 \mathrm{~Hz}), 7.04-7.11(\mathrm{~m}, 1 \mathrm{H}), 6.96-7.02(\mathrm{~m}$, $1 \mathrm{H}), 4.30-4.37(\mathrm{~m}, 0.5 \mathrm{H}), 4.16-4.23(\mathrm{~m}, 0.5 \mathrm{H}), 3.47-3.61(\mathrm{~m}, 1 \mathrm{H}), 3.28-3.41(\mathrm{~m}, 1 \mathrm{H}), 3.05-3.17(\mathrm{~m}, 1 \mathrm{H}), 2.82(\mathrm{dd}$, $\left.0.5 \mathrm{H}, J_{1}=9.8 \mathrm{~Hz}, J_{2}=13.9 \mathrm{~Hz}\right), 2.65\left(\mathrm{dd}, 0.5 \mathrm{H}, J_{1}=9.8 \mathrm{~Hz}, J_{2}=13.9 \mathrm{~Hz}\right), 2.32-2.44(\mathrm{~m}, 2 \mathrm{H}), 1.66-2.00(\mathrm{~m}, 4 \mathrm{H}), 1.09-$ $1.19(\mathrm{~m}, 3 \mathrm{H})$; LRMS(CI) (m/z, relative intensity) : $281\left([\mathrm{M}+\mathrm{H}]^{+}, 100\right), 227(7), 150(26)$.

1-[(S)-2-(1 H-Indol-3-ylmethyl)-pyrrolidin-1-yl]-3-phenylpropynone (1r). Synthesized from 3-phenylpropynoic acid $336 \mathrm{mg}$ and amine $8401 \mathrm{mg}$. Eluent DCM / $\mathrm{Et}_{2} \mathrm{O}(4 / 1)\left(R_{f} 0.25\right.$ and $\left.R_{f} 0.39\right)$. Yield: $604 \mathrm{mg}(92 \%)$. (mixture of rotamers 1/1) ${ }^{1} \mathbf{H}$ NMR (DMSO- $\left.d_{6}, 300 \mathrm{MHz}\right): \delta 10.87(\mathrm{bs}, 1 \mathrm{H}), 7.60-7.70(\mathrm{~m}, 1.5 \mathrm{H}), 7.42-7.58(\mathrm{~m}, 4.5 \mathrm{H})$, 7.28$7.37(\mathrm{~m}, 1 \mathrm{H}), 7.17(\mathrm{~s}, 1 \mathrm{H}), 6.95-7.11(\mathrm{~m}, 1.5 \mathrm{H}), 6.68-6.77(\mathrm{~m}, 0.5 \mathrm{H}), 4.41-4.53(\mathrm{~m}, 0.5 \mathrm{H}), 4.22-4.33(\mathrm{~m}, 0.5 \mathrm{H}), 3.60-$ $3.76(\mathrm{~m}, 1 \mathrm{H}), 3.37-3.48(\mathrm{~m}, 1 \mathrm{H}), 3.11-3.23(\mathrm{~m}, 1 \mathrm{H}), 2.82-2.95(\mathrm{~m}, 0.5 \mathrm{H}), 2.65-2.77(\mathrm{~m}, 0.5 \mathrm{H}), 1.64-2.08(\mathrm{~m}, 4 \mathrm{H})$; LRMS(Cl) (m/z, relative intensity) : $329\left([\mathrm{M}+\mathrm{H}]^{+}, 100\right), 227(15), 198(25)$.

\section{Synthesis of propiolic acid amides 11,m:}

Propynoic acid benzyl-[2-(1 H-indol-3-yl)-ethyl]-amide (1). To a solution of amide 12a $803 \mathrm{mg}(2.14 \mathrm{mmol})$ in THF (10 mL) cooled to $0{ }^{\circ} \mathrm{C}$, a solution of TBAF in THF (1M) $2.14 \mathrm{~mL}$ (1 equiv) was added. The reaction was kept with stirring at $0{ }^{\circ} \mathrm{C}$ for 30 min followed by dilution with $\mathrm{Et}_{2} \mathrm{O}$. The organic layer was washed with $\mathrm{H}_{2} \mathrm{O}$, brine, dried $\left(\mathrm{MgSO}_{4}\right)$, concentrated and applied to a column of silica gel as a solution in DCM. Elution with DCM / Et $2 \mathrm{O}(20: 1)\left(R_{f}\right.$ 0.25 ) afforded pure $11427 \mathrm{mg}(66 \%)$. (mixture of rotamers 1/ 1) ${ }^{1} \mathbf{H}$ NMR (DMSO- $\left.d_{6}, 300 \mathrm{MHz}\right): \delta 10.85$ (bs, $1 \mathrm{H}$ ), 7.21-7.55 (m, 7H), 6.92-7.16 (m, 3H), $4.76(\mathrm{~s}, 1 \mathrm{H}), 4.60(\mathrm{~s}, 1 \mathrm{H}), 4.56(\mathrm{~s}, 0.5 \mathrm{H}), 4.54(\mathrm{~s}, 0.5 \mathrm{H}), 3.67-3.75(\mathrm{~m}, 1 \mathrm{H}), 3.42-$ 
$3.51(\mathrm{~m}, 1 \mathrm{H}), 2.92-3.01(\mathrm{~m}, 1 \mathrm{H}), 2.79-2.87(\mathrm{~m}, 1 \mathrm{H})$; LRMS(Cl) (m/z, relative intensity) : $303\left([\mathrm{M}+\mathrm{H}]^{+}, 100\right), 172(5)$, 144 (28), 143 (34), 91 (5).

Propynoic acid [2-(1H-indol-3-yl)-ethyl]-methylamide (1m). Synthesized analogously to 1I: from $12 \mathrm{~b} 640 \mathrm{mg}$ $(2.14 \mathrm{mmol})$. Eluent DCM / Et ${ }_{2} \mathrm{O}(4 / 1)\left(R_{f} 0.30\right)$. Yield: $439 \mathrm{mg}(92 \%)$. (mixture of rotamers $\left.~ 13 / 7\right){ }^{1} \mathbf{H}$ NMR (DMSO$d_{6}, 300 \mathrm{MHz}$ ) : $\delta 10.85$ (bs, 1H), $7.56(\mathrm{~d}, 1 \mathrm{H}, J=7.9 \mathrm{~Hz}), 7.34$ (d, $\left.1 \mathrm{H}, J=7.9 \mathrm{~Hz}\right), 7.15(\mathrm{~s}, 1 \mathrm{H}), 7.07(\mathrm{t}, 1 \mathrm{H}, J=7.9$ $\mathrm{Hz}), 6.98(\mathrm{t}, 1 \mathrm{H}, J=7.9 \mathrm{~Hz}), 4.51(\mathrm{~s}, 0.35 \mathrm{H}), 4.39(\mathrm{~s}, 0.65 \mathrm{H}), 3.74-3.83(\mathrm{~m}, 1.3 \mathrm{H}), 3.54-3.62(\mathrm{~m}, 0.7 \mathrm{H}), 3.12(\mathrm{~s}$, 1.05H), 2.93-3.02 (m, 1.3H), 2.84-2.92 (m, 2.65H); LRMS(Cl) (m/z, relative intensity) : $227\left([\mathrm{M}+\mathrm{H}]^{+}, 100\right), 172(12)$, $144(81), 143(34), 130(12)$.

\section{Synthesis of tetrolic acid amides 1n,o,s:}

2-Butynoic acid benzyl-\{2-[1-(toluene-4-sulfonyl)-1H-indol-3-yl]-ethyl\}-amide (1n). To a mixture of 1a 316 $\mathrm{mg}(1 \mathrm{mmol}), \mathrm{Bu}_{4} \mathrm{NHSO}_{4} 34 \mathrm{mg}(10 \mathrm{~mol} \%)$ and powdered $\mathrm{NaOH} 200 \mathrm{mg}$ (5 equiv) in DCM (8 mL) a solution of $\mathrm{TsCl}$ $478 \mathrm{mg}$ (2.5 equiv) in DCM (5 mL) was added dropwise at rt. After $2 \mathrm{~h}$ the reaction mixture was diluted with DCM and washed successively with $\mathrm{H}_{2} \mathrm{O}$ and brine, dried $\left(\mathrm{MgSO}_{4}\right)$, concentrated and applied to a column of silica gel as a solution in DCM. Elution with DCM / $\mathrm{Et}_{2} \mathrm{O}(20: 1)\left(R_{f} 0.38\right)$ afforded pure $1 \mathrm{n} 438 \mathrm{mg}(93 \%)$. (mixture of rotamers $\sim 1 /$ 1) ${ }^{1} \mathrm{H}$ NMR $\left(\mathrm{CDCl}_{3}, 300 \mathrm{MHz}\right): \delta$ 7.92-8.01 $(\mathrm{m}, 1 \mathrm{H}), 7.69-7.78(\mathrm{~m}, 1 \mathrm{H})$, 7.37-7.45 $(\mathrm{m}, 1 \mathrm{H})$, 7.11-7.35 $(\mathrm{m}, 10 \mathrm{H})$, , 4.56$4.62(\mathrm{~m}, 2 \mathrm{H}), 3.69-3.77(\mathrm{~m}, 1 \mathrm{H}), 3.48-3.57(\mathrm{~m}, 1 \mathrm{H}), 2.80-2.92(\mathrm{~m}, 2 \mathrm{H}), 2.27-2.35(\mathrm{~m}, 3 \mathrm{H}), 2.01(1.5 \mathrm{H}), 1.55(\mathrm{~s}, 1.5 \mathrm{H})$.

2-Butynoic acid [2-(1-acetyl-1H-indol-3-yl)-ethyl]-benzylamide (10). Synthesized analogously to $1 \mathrm{n}$ : from $1 \mathrm{a}$ $949 \mathrm{mg}(3 \mathrm{mmol})$ and $\mathrm{AcCl} 533 \mu \mathrm{L}$ (2.5 equiv). Eluent DCM / $\mathrm{Et}_{2} \mathrm{O}(20 / 1)\left(R_{f} 0.17\right)$. Yield: $879 \mathrm{mg}(82 \%)$. (mixture of rotamers 1/ 1) ${ }^{1} \mathrm{H}$ NMR (DMSO- $\left.d_{6}, 300 \mathrm{MHz}\right): \delta 8.26-8.35(\mathrm{~m}, 1 \mathrm{H}), 7.50-7.65(\mathrm{~m}, 2 \mathrm{H}), 7.22-7.42(\mathrm{~m}, 7 \mathrm{H}), 4.81(\mathrm{~s}$, $1.5 \mathrm{H}), 4.63(\mathrm{~s}, 1 \mathrm{H}), 3.72-3.80(\mathrm{~m}, 1 \mathrm{H}), 3.47-3.56(\mathrm{~s}, 1 \mathrm{H}), 2.91-3.00(\mathrm{~m}, 1 \mathrm{H}), 2.78-2.87(\mathrm{~m}, 1 \mathrm{H}), 2.59(\mathrm{~s}, 3 \mathrm{H}), 2.02(\mathrm{~s}$, 1.5H), 1.79 (s, 1.5H); LRMS(EI) (m/z, relative intensity) : $392\left(\mathrm{M}^{+*}, 13\right), 185(100), 143(51), 130(32), 120(26), 91$ (45), 67 (27).

(S)-2-[Benzyl-(1-oxo-but-2-ynyl)-amino]-3-(1H-indol-3-yl)-propionic acid methyl ester (1s). To a mixture of

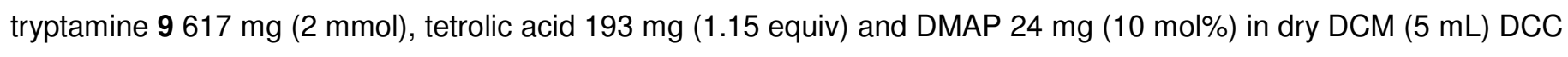
$475 \mathrm{mg}$ ( 1.15 equiv) was added in one portion. The reaction was left with stirring at $\mathrm{rt}$ for approximately $12 \mathrm{~h}$ The subsequently formed precipitate of $\mathrm{N}, \mathrm{N}^{\prime}$-dicyclohexylurea was filtered off and washed well with $\mathrm{Et}_{2} \mathrm{O}$. The organic layer was washed with successively with $\mathrm{HCl}(1 \mathrm{M}), \mathrm{NaHCO}_{3}$ (sat), brine, dried $\left(\mathrm{MgSO}_{4}\right)$, concentrated with silica and applied to a silica gel column. Elution with DCM / $\mathrm{Et}_{2} \mathrm{O}(4: 1)\left(R_{f} 0.41\right.$ and $\left.R_{f} 0.50\right)$ afforded pure $1 \mathrm{~s} 419 \mathrm{mg}(56 \%)$. (mixture of rotamers 1/1) ${ }^{1} \mathrm{H}$ NMR (DMSO- $d_{6}, 300 \mathrm{MHz}$ ) : $\delta 10.86$ (bs, $1 \mathrm{H}$ ), 7.60 (d, 0.5H, J = 7.8 Hz), 6.91-7.40 $(\mathrm{m}, 9.5 \mathrm{H}), 5.38\left(\mathrm{dd}, 0.5 \mathrm{H}, J_{1}=6.2 \mathrm{~Hz}, J_{2}=9.2 \mathrm{~Hz}\right), 4.68(\mathrm{~d}, 0.5 \mathrm{H}, J=16.0 \mathrm{~Hz}), 4.60(\mathrm{~d}, 0.5 \mathrm{H}, J=16.0 \mathrm{~Hz}), 4.51$ (dd, $\left.0.5 \mathrm{H}, J_{1}=6.2 \mathrm{~Hz}, J_{2}=9.2 \mathrm{~Hz}\right), 4.42(\mathrm{~d}, 0.5 \mathrm{H}, J=16.0 \mathrm{~Hz}), 4.07(\mathrm{~d}, 0.5 \mathrm{H}, J=16.0 \mathrm{~Hz}), 3.31-3.45(\mathrm{~m}, 4 \mathrm{H}), 3.09-3.23$ $(\mathrm{m}, 1 \mathrm{H}), 1.96(\mathrm{~s}, 1.5 \mathrm{H}), 1.72(\mathrm{~s}, 1.5 \mathrm{H})$; LRMS(Cl) (m/z, relative intensity) : $375\left([\mathrm{M}+\mathrm{H}]^{+}, 100\right), 343(17), 201(57), 130$ (14). 


\section{Synthesis of propynoic acid amides $1 \mathrm{u}, \mathbf{v}$ :}

A mixture of aminoalcohol $10561 \mathrm{mg} \mathrm{(2} \mathrm{mmol),} \mathrm{imidazole} 204 \mathrm{mg}$ (1.5 equiv) and TIPSCI $470 \mu \mathrm{L}$ (1.1 equiv) in dry DMF was heated with stirring at $70{ }^{\circ} \mathrm{C}$ for approximately $5 \mathrm{~h}$. Then the solvent was removed in vacuo and the residue was partitioned between $\mathrm{Et}_{2} \mathrm{O}$ and $\mathrm{H}_{2} \mathrm{O}$. The organic layer was washed excessively with $\mathrm{H}_{2} \mathrm{O}$, then with brine and dried $\left(\mathrm{Na}_{2} \mathrm{SO}_{4}\right)$. Concentration afforded $~ 920 \mathrm{mg}$ of crude O-TIPS protected aminoalcohol which was dissolved in dry DCM (5 mL). To this solution 3 substituted 2-propynoic acid 11 (1.2 equiv), DMAP $48 \mathrm{mg}$ (20 mol\%) was added followed by DCC $495 \mathrm{mg}$ (1.2 equiv). The reaction was left with stirring at $\mathrm{rt}$ for approximately $12 \mathrm{~h}$ The subsequently formed precipitate of $\mathrm{N}, \mathrm{N}^{\prime}$-dicyclohexylurea was filtered off and washed well with $\mathrm{Et}_{2} \mathrm{O}$. The organic layer was washed with $\mathrm{H}_{2} \mathrm{O}$, brine and dried $\left(\mathrm{MgSO}_{4}\right)$, Pure amides $\mathbf{1} \mathbf{u}, \mathbf{v}$ were isolated by column chromatography on silica gel.

2-Butynoic acid (S)-benzyl-[2-(1 H-indol-3-yl)-1-triisopropylsilanyloxymethylethyl]-amide (1u). Synthesized from tetrolic acid $202 \mathrm{mg}$. Eluent DCM / $\mathrm{Et}_{2} \mathrm{O}(20 / 1)\left(R_{f} 0.37\right.$ and $\left.R_{f} 0.44\right)$. Yield: $578 \mathrm{mg}(57 \%)$. (mixture of rotamers $7 / 3$ ) ${ }^{1} \mathrm{H}$ NMR (DMSO- $\left.d_{6}, 300 \mathrm{MHz}\right): \delta 10.79$ (bs, $\left.1 \mathrm{H}\right), 7.55$ (d, 0.7H, $J=7.5 \mathrm{~Hz}$ ), 7.15-7.38 (m, 6.3H), 6.87-7.11 $(\mathrm{m}, 3 \mathrm{H}), 4.84-5.03(\mathrm{bs}, 0.7 \mathrm{H}), 4.45-4.74(\mathrm{~m}, 2 \mathrm{H}), 4.21-4.39(\mathrm{bs}, 0.3 \mathrm{H}), 3.52-3.81(\mathrm{~m}, 2 \mathrm{H}), 2.78-3.06(\mathrm{~m}, 2 \mathrm{H}), 1.92(\mathrm{~s}$, 0.9H), $1.71(2.1 \mathrm{H}), 0.74-1.09(\mathrm{~m}, 21 \mathrm{H})$; LRMS(Cl) $\left(\mathrm{m} / \mathrm{z}\right.$, relative intensity) : $503\left([\mathrm{M}+\mathrm{H}]^{+}, 15\right), 459(20), 357(8), 329$ (100), $286(11)$.

2-Octynoic acid (S)-benzyl-[2-(1 H-indol-3-yl)-1-triisopropylsilanyloxymethylethyl]-amide (1v). Synthesized from 2-octynoic acid $336 \mathrm{mg}$. Eluent DCM / $\mathrm{Et}_{2} \mathrm{O}(20 / 1)\left(R_{f} 0.34\right.$ and $\left.R_{f} 0.44\right)$. Yield: $754 \mathrm{mg}(67 \%)$. (mixture of rotamers $7 / 3)^{1} \mathrm{H}$ NMR (DMSO- $\left.d_{6}, 300 \mathrm{MHz}\right): \delta 7.53(\mathrm{~d}, 0.7 \mathrm{H}, J=7.4 \mathrm{~Hz}), 7.15-7.37(\mathrm{~m}, 6.3 \mathrm{H}), 6.87-7.10(\mathrm{~m}, 3 \mathrm{H})$, 4.80-4.99 (bs, 0.7H), 4.46-4.77 (m, 2H), 4.27-4.44 (bs, 0.3H), 3.53-3.83 (m, 2H), 2.77-3.07 (m, 2H), 2.25-2.33 (m, 0.6H), 2.02-2.13 (m, 1.4H), 1.11-1.39 (m, 6H), 0.74-1.02 (m, 24H); LRMS(Cl) (m/z, relative intensity) : $559\left([\mathrm{M}+\mathrm{H}]^{+}\right.$, 31), 515 (34), 385 (96), 329 (100), 286 (18).

\section{General procedure for $\mathrm{Hg}(\mathrm{OTf})_{2}$ catalyzed cyclization:}

In ${ }^{1} \mathrm{H}$ NMR spectra of indoloazocinones 2a-p, recorded at $298 \mathrm{~K}$, signals of the 8-membered ring protons are often broadened due to the ring flipping.

$\mathrm{Hg}(\mathrm{OTf})_{2}(5-30 \mathrm{~mol} \%)$ and propynoic acid amide 1a-v $(0.6 \mathrm{mmol})$ were loaded into the microwave instrument vial followed by dry DCM $(2.4 \mathrm{~mL})$. The vial was sealed and irradiated with stirring at a ceiling temperature of 80 $120^{\circ} \mathrm{C}$ at 60-120 $\mathrm{W}$ maximum power level for 15-50 min. Upon completion of the reaction time the vial was cooled with a stream of air. The precipitate of $\mathrm{Hg}(\mathrm{OTf})_{2}$ was filtered off through a plug of Celite $\AA$ and washed well with DCM until TLC analysis indicated absence of product in the flowing filtrate. The DCM layer was washed with $\mathrm{NaHCO}_{3}$ (sat.), brine, dried $\left(\mathrm{MgSO}_{4}\right)$ and concentrated with silica. Pure indoloazocinones 2a-p were isolated by column chromatography on silica gel.

3-Benzyl-6-methyl-1,2,3,7-tetrahydro-azocino[5,4-b]indol-4-one (2a). Synthesized from amide 1a $190 \mathrm{mg}$ with $15 \mathrm{mg} \mathrm{Hg}(\mathrm{OTf})_{2}(5 \mathrm{~mol} \%)$. MW irradiation - $60 \mathrm{~W}, 80 \stackrel{\circ}{\circ} \mathrm{C}, 15 \mathrm{~min}$. Eluent DCM / EtOAc (1/1) $\left(R_{f} 0.46\right)$. Yield: 162 
mg (85\%). ${ }^{1} \mathbf{H}$ NMR (DMSO- $\left.d_{6}, 400 \mathrm{MHz}\right): \delta 10.86(\mathrm{~s}, 1 \mathrm{H}), 7.39(\mathrm{~d}, 1 \mathrm{H}, J=7.7 \mathrm{~Hz}), 7.31(\mathrm{~d}, 1 \mathrm{H}, J=7.7 \mathrm{~Hz}), 7.21-$ $7.29(\mathrm{~m}, 5 \mathrm{H}), 7.13(\mathrm{t}, 1 \mathrm{H}, J=7.5 \mathrm{~Hz}), 7.00(\mathrm{t}, 1 \mathrm{H}, J=7.5 \mathrm{~Hz}), 6.00(\mathrm{~d}, 1 \mathrm{H}, J=1.1 \mathrm{~Hz}), 4.31-4.80(\mathrm{bs}, 2 \mathrm{H}), 3.51-4.14$ (bs, 2H), 2.89-3.24 (m, 4H), $2.25(\mathrm{~d}, 3 \mathrm{H}, J=1.1 \mathrm{~Hz}) ;{ }^{13} \mathrm{C}$ NMR (DMSO- $\left.d_{6}, 100 \mathrm{MHz}\right): \delta 168.50,138.13,136.24$, 134.25, 132.34, 128.88, 128.83, 128.12, 127.51, 122.78, 122.61, 119.17, 118.90, 111.32, 108.89, 47.18, 44.96, 24.48, 24.22; HRMS(EI) calcd. for $\mathrm{C}_{21} \mathrm{H}_{20} \mathrm{~N}_{2} \mathrm{O}: \mathrm{m} / \mathrm{z} 316.1576$, found $\mathrm{m} / \mathrm{z} 316.1571$

3-Benzyl-6-propyl-1,2,3,7-tetrahydro-azocino[5,4-b]indol-4-one (2b). Synthesized from amide 1 b $207 \mathrm{mg}$ with $15 \mathrm{mg} \mathrm{Hg}(\mathrm{OTf})_{2}(5 \mathrm{~mol} \%)$. MW irradiation - $60 \mathrm{~W}, 80 \stackrel{\circ}{\circ} \mathrm{C}, 15 \mathrm{~min}$. Eluent $\mathrm{DCM} / \mathrm{Et}_{2} \mathrm{O}(4 / 1)\left(R_{f} 0.29\right)$. Yield: 169 mg (82\%). ${ }^{1} \mathrm{H}$ NMR (DMSO- $\left.d_{6}, 400 \mathrm{MHz}\right): \delta 10.84(\mathrm{~s}, 1 \mathrm{H}), 7.39(\mathrm{~d}, 1 \mathrm{H}, J=7.7 \mathrm{~Hz}), 7.32(\mathrm{~d}, 1 \mathrm{H}, J=7.7 \mathrm{~Hz}), 7.21$ $7.29(\mathrm{~m}, 5 \mathrm{H}), 7.13(\mathrm{t}, 1 \mathrm{H}, J=7.7 \mathrm{~Hz}), 7.00(\mathrm{t}, 1 \mathrm{H}, J=7.7 \mathrm{~Hz}), 6.00(\mathrm{~s}, 1 \mathrm{H}), 4.24-4.97$ (bs, 2H), 3.47-4.21 (bs, 2H), 2.85-3.30 (bs, 2H), 2.46-2.83 (bs, 2H), 1.31-1.43 (m, 2H), 0.88 (t, 3H, $J=7.3 \mathrm{~Hz}$ ); ${ }^{13} \mathrm{C}$ NMR (DMSO-d, $\left.100 \mathrm{MHz}\right): \delta$ $168.71,138.60,138.15,136.30,131.42,128.96,128.80,128.11,127.48,122.61,122.34,119.04,118.74,111.30$, 109.50, 47.17, 45.16, 39.24, 24.33, 21.23, 13.93; HRMS(EI) calcd. for $\mathrm{C}_{23} \mathrm{H}_{24} \mathrm{~N}_{2} \mathrm{O}: \mathrm{m} / \mathrm{z} 344.1889$, found m/z 316.1885

3-(4-Methoxybenzyl)-6-methyl-1,2,3,7-tetrahydro-azocino[5,4-b]indol-4-one (2c). Synthesized from amide 1c $208 \mathrm{mg}$ with $15 \mathrm{mg} \mathrm{Hg}(\mathrm{OTf})_{2}$ (5 mol\%). MW irradiation - $60 \mathrm{~W}, 80 \stackrel{\circ}{\mathrm{C}}, 15 \mathrm{~min}$. Eluent DCM / EtOAc $(1 / 1)\left(R_{f} 0.34\right)$. Yield: $123 \mathrm{mg}(59 \%) .{ }^{1} \mathrm{H}$ NMR (DMSO- $\left.d_{6}, 400 \mathrm{MHz}\right): \delta 10.85(\mathrm{~s}, 1 \mathrm{H}), 7.38(\mathrm{~d}, 1 \mathrm{H}, J=7.6 \mathrm{~Hz}), 7.31(\mathrm{~d}, 1 \mathrm{H}, J=7.6$ $\mathrm{Hz}), 7.19(\mathrm{~d}, 2 \mathrm{H}, J=8.5 \mathrm{~Hz}), 7.13(\mathrm{t}, 1 \mathrm{H}, J=7.6 \mathrm{~Hz}), 6.99(\mathrm{t}, 1 \mathrm{H}, J=7.6 \mathrm{~Hz}), 6.82(\mathrm{~d}, 2 \mathrm{H}, J=8.5 \mathrm{~Hz}), 5.97(\mathrm{~d}, 1 \mathrm{H}, J=$ $1.3 \mathrm{~Hz}$ ), 4.17-4.85 (bs, 2H), 3.51-3.99 (m, 5H), 2.87-3.25 (bs, 2H), 2.25 (d, $1 \mathrm{H}, J=1.3 \mathrm{~Hz}$ ); ${ }^{13} \mathrm{C}$ NMR (DMSO- $d_{6}, 100$ $\mathrm{MHz}): \delta 168.35,158.901,136.21,134.12,132.34,130.05,129.65,128.89,122.75,122.70,119.13,118.90,114.25$, 111.30, 108.90, 55.49, 46.41, 44.59, 24.40, 24.19; HRMS(EI) calcd. for $\mathrm{C}_{22} \mathrm{H}_{22} \mathrm{~N}_{2} \mathrm{O}_{2}: \mathrm{m} / \mathrm{z} 346.1681$, found m/z 316.1687

3,6-Dimethyl-1,2,3,7-tetrahydro-azocino[5,4-b]indol-4-one (2d). Synthesized from amide 1e $144 \mathrm{mg}$ with 15 $\mathrm{mg} \mathrm{Hg}(\mathrm{OTf})_{2}$ (5 mol\%). MW irradiation - $60 \mathrm{~W}, 80{ }^{\circ} \mathrm{C}, 20 \mathrm{~min}$. Eluent DCM / MeCN (1/1) $\left(R_{f} 0.31\right)$. Yield: $124 \mathrm{mg}$ (86\%). ${ }^{1} \mathrm{H}$ NMR (DMSO- $\left.d_{6}, 400 \mathrm{MHz}\right): \delta 10.82(\mathrm{~s}, 1 \mathrm{H}), 7.47(\mathrm{~d}, 1 \mathrm{H}, J=7.8 \mathrm{~Hz}), 7.32(\mathrm{~d}, 1 \mathrm{H}, J=7.8 \mathrm{~Hz}), 7.13(\mathrm{t}, 1 \mathrm{H}, J$ $=7.8 \mathrm{~Hz}), 7.02(\mathrm{t}, 1 \mathrm{H}, J=7.8 \mathrm{~Hz}), 5.91(\mathrm{~d}, 1 \mathrm{H}, J=1.1 \mathrm{~Hz}), 3.66-4.11(\mathrm{bs}, 2 \mathrm{H}), 3.01-3.22(\mathrm{bs}, 2 \mathrm{H}), 2.88(\mathrm{~s}, 3 \mathrm{H}), 2.25$ $\left(\mathrm{d}, 3 \mathrm{H}, J=1.1 \mathrm{~Hz}\right.$ ); ${ }^{13} \mathrm{C}$ NMR (DMSO-d, $100 \mathrm{MHz}$ ) : $\delta 168.14,136.21,133.94,132.31,128.94,122.90,122.76$, 119.19, 118.90, 111.30, 108.70, 47.42, 32.07, 24.36, 24.12; HRMS(EI) calcd. for $\mathrm{C}_{15} \mathrm{H}_{16} \mathrm{~N}_{2} \mathrm{O}: \mathrm{m} / \mathrm{z} 240.1263$, found $\mathrm{m} / \mathrm{z} 316.1262$

6-Ethyl-3-methyl-1,2,3,7-tetrahydro-azocino[5,4-b]indol-4-one (2e). Synthesized from amide $1 \mathrm{f} 153 \mathrm{mg}$ with $15 \mathrm{mg} \mathrm{Hg}(\mathrm{OTf})_{2}(5 \mathrm{~mol} \%)$. MW irradiation - $60 \mathrm{~W}, 80 \stackrel{\circ}{\circ}$, $20 \mathrm{~min}$. Eluent DCM / MeCN (1/ 1) $\left(R_{f} 0.30\right)$. Yield: $102 \mathrm{mg}$ (67\%). ${ }^{1} \mathrm{H}$ NMR (DMSO- $\left.d_{6}, 400 \mathrm{MHz}\right): \delta 10.81(\mathrm{~s}, 1 \mathrm{H}), 7.46(\mathrm{~d}, 1 \mathrm{H}, J=7.8 \mathrm{~Hz}), 7.29(\mathrm{~d}, 1 \mathrm{H}, J=7.8 \mathrm{~Hz}), 7.12(\mathrm{t}, 1 \mathrm{H}, J$ $=7.8 \mathrm{~Hz}), 7.00(\mathrm{t}, 1 \mathrm{H}, J=7.8 \mathrm{~Hz}), 5.88(\mathrm{~s}, 1 \mathrm{H}), 3.48-4.42(\mathrm{bs}, 2 \mathrm{H}), 2.94-3.29(\mathrm{bs}, 2 \mathrm{H}), 2.86(\mathrm{~s}, 3 \mathrm{H}), 2.60(\mathrm{q}, 2 \mathrm{H}, J=$ $7.5 \mathrm{~Hz}$ ), 1.01 (t, 3H, $J=7.5 \mathrm{~Hz}$ ); ${ }^{13} \mathrm{C}$ NMR (DMSO- $d_{6}, 100 \mathrm{MHz}$ ) : $\delta 168.41,139.88,136.22,131.52,128.98,122.62$, 121.34, 119.09, 118.76, 111.29, 109.27, 47.48, 32.02, 30.19, 24.18, 13.30: HRMS(EI) calcd. for $\mathrm{C}_{16} \mathrm{H}_{18} \mathrm{~N}_{2} \mathrm{O}: \mathrm{m} / \mathrm{z}$ 254.1419 , found $\mathrm{m} / \mathrm{z} 254.1431$

3-Methyl-6-propyl-1,2,3,7-tetrahydro-azocino[5,4-b]indol-4-one (2f). Synthesized from amide $1 \mathrm{~g} 161 \mathrm{mg}$ with $15 \mathrm{mg} \mathrm{Hg}(\mathrm{OTf})_{2}(5 \mathrm{~mol} \%)$. MW irradiation - $60 \mathrm{~W}, 80^{\circ} \mathrm{C}, 20 \mathrm{~min}$. Eluent DCM / MeCN (1/ 1) $\left(R_{f} 0.36\right)$. Yield: 121 $\mathrm{mg}(75 \%) .{ }^{1} \mathrm{H}$ NMR (DMSO- $\left.d_{6}, 400 \mathrm{MHz}\right): \delta 10.81(\mathrm{~s}, 1 \mathrm{H}), 7.45(\mathrm{~d}, 1 \mathrm{H}, J=7.7 \mathrm{~Hz}), 7.29(\mathrm{~d}, 1 \mathrm{H}, J=7.7 \mathrm{~Hz}), 7.11$ (t, 
$1 \mathrm{H}, J=7.7 \mathrm{~Hz}$ ), 6.99 (t, $1 \mathrm{H}, J=7.7 \mathrm{~Hz}$ ), 5.89 (s, 1H), 3.46-4.34 (bs, 2H), 2.91-3.30 (bs, 2H), 2.86 (s, 3H), 2.47-2.72 (bs, 2H), 1.28-1.41 (m, 2H), 0.87 (t, 3H, $J=7.4 \mathrm{~Hz}$ ); ${ }^{13} \mathrm{C}$ NMR (DMSO-d, $100 \mathrm{MHz}$ ) : $\delta 168.33,138.32,136.22$, 131.34, 128.99, 122.59, 119.08, 118.74, 111.29, 109.27, 47.55, 39.17, 32.01, 24.16, 21.24, 13.93; HRMS(EI) calcd. for $\mathrm{C}_{17} \mathrm{H}_{20} \mathrm{~N}_{2} \mathrm{O}: \mathrm{m} / \mathrm{z} 268.1576$, found $\mathrm{m} / \mathrm{z} 268.11568$

6-Isopropyl-3-methyl-1,2,3,7-tetrahydro-azocino[5,4-b]indol-4-one (2g). Synthesized from amide $1 \mathrm{~h} 161 \mathrm{mg}$ with $45 \mathrm{mg} \mathrm{Hg}(\mathrm{OTf})_{2}$ (15 mol\%). MW irradiation - $90 \mathrm{~W}, 100 \stackrel{\circ}{\circ} \mathrm{C}, 20 \mathrm{~min}$. Eluent DCM / EtOAc $(1 / 1)\left(R_{f} 0.51\right)$. Yield: $127 \mathrm{mg}(79 \%) .{ }^{1} \mathrm{H}$ NMR (DMSO- $\left.d_{6}, 400 \mathrm{MHz}, 363 \mathrm{~K}\right): \delta 10.44(\mathrm{~s}, 1 \mathrm{H}), 7.46(\mathrm{~d}, 1 \mathrm{H}, J=7.7 \mathrm{~Hz}), 7.31(\mathrm{~d}, 1 \mathrm{H}, J=7.7$ $\mathrm{Hz}), 7.10(\mathrm{t}, 1 \mathrm{H}, J=7.7 \mathrm{~Hz}), 7.00(\mathrm{t}, 1 \mathrm{H}, J=7.7 \mathrm{~Hz}), 5.84(\mathrm{~d}, 1 \mathrm{H}, J=1.3 \mathrm{~Hz}), 3.89$ (t, 2H, $J=6.1 \mathrm{~Hz}), 3.12(\mathrm{t}, 2 \mathrm{H}, J=$ $6.1 \mathrm{~Hz}$ ), 2.97 (dseptet, $1 \mathrm{H}, J_{1}=1.3 \mathrm{~Hz}, J_{2}=6.7 \mathrm{~Hz}$ ), $2.86(\mathrm{~s}, 3 \mathrm{H}), 1.14\left(\mathrm{~d}, 6 \mathrm{H}, J=6.7 \mathrm{~Hz}\right.$ ); ${ }^{13} \mathrm{C}$ NMR (DMSO- $d_{6}, 100$ $\mathrm{MHz}, 363 \mathrm{~K}): \delta 168.94,144.68,136.63,132.50,129.15,122.29,120.33,119.05,118.38,111.35,108.97,47.78$, 33.55, 31.94, 24.01, 21.96; HRMS(EI) calcd. for $\mathrm{C}_{17} \mathrm{H}_{20} \mathrm{~N}_{2} \mathrm{O}: \mathrm{m} / \mathrm{z} 268.1576$, found $\mathrm{m} / \mathrm{z} 268.11572$

3-Methyl-6-phenyl-1,2,3,7-tetrahydro-azocino[5,4-b]indol-4-one (2h). Synthesized from amide 1j $181 \mathrm{mg}$ with $60 \mathrm{mg} \mathrm{Hg}(\mathrm{OTf})_{2}$ (20 mol\%). MW irradiation - $90 \mathrm{~W}, 100 \stackrel{\circ}{\circ} \mathrm{C}, 20 \mathrm{~min}$. Eluent DCM / MeCN (3/1) $\left(R_{f} 0.37\right)$. Yield: $111 \mathrm{mg}(61 \%) .{ }^{1} \mathrm{H}$ NMR (DMSO- $\left.d_{6}, 400 \mathrm{MHz}, 363 \mathrm{~K}\right): \delta 10.02(\mathrm{~s}, 1 \mathrm{H}), 7.54(\mathrm{~d}, 1 \mathrm{H}, J=7.9 \mathrm{~Hz}), 7.33-7.43(\mathrm{~m}, 5 \mathrm{H})$, $7.26(\mathrm{~d}, 1 \mathrm{H}, J=7.9 \mathrm{~Hz}), 7.11(\mathrm{t}, 1 \mathrm{H}, J=7.9 \mathrm{~Hz}), 7.03(\mathrm{t}, 1 \mathrm{H}, J=7.9 \mathrm{~Hz}), 6.32(\mathrm{~s}, 1 \mathrm{H}), 3.99(\mathrm{t}, 2 \mathrm{H}, J=6.2 \mathrm{~Hz}), 3.27(\mathrm{t}$, $2 \mathrm{H}, J=6.2 \mathrm{~Hz}$ ), $2.93(\mathrm{~s}, 3 \mathrm{H}) ;{ }^{13} \mathrm{C}$ NMR (DMSO-d $\left., 100 \mathrm{MHz}, 363 \mathrm{~K}\right): \delta 168.47,140.58,138.20,136.91,130.80$, 129.05, 128.76, 128.71, 128.22, 124.17, 122.67, 119.18, 118.56, 111.67, 110.93, 47.91, 32.09, 24.15; HRMS(EI) calcd. for $\mathrm{C}_{20} \mathrm{H}_{18} \mathrm{~N}_{2} \mathrm{O}: \mathrm{m} / \mathrm{z} 302.1419$, found $\mathrm{m} / \mathrm{z} 302.1425$

3-Methyl-6-(3,4,5-trimethoxyphenyl)-1,2,3,7-tetrahydro-azocino[5,4-b]indol-4-one (2i). Synthesized from amide 1k $235 \mathrm{mg}$ with $90 \mathrm{mg} \mathrm{Hg}(\mathrm{OTf})_{2}$ (30 mol\%). MW irradiation - $120 \mathrm{~W}, 120 \stackrel{\circ}{\circ} \mathrm{C}, 50 \mathrm{~min}$. Eluent DCM / MeCN (3/ 1) $\left(R_{f} 0.23\right)$. Yield: $94 \mathrm{mg}(40 \%) .{ }^{1} \mathrm{H}$ NMR (DMSO- $\left.d_{6}, 400 \mathrm{MHz}, 363 \mathrm{~K}\right): \delta 10.08(\mathrm{~s}, 1 \mathrm{H}), 7.54(\mathrm{~d}, 1 \mathrm{H}, J=7.7 \mathrm{~Hz}), 7.29$ $(\mathrm{d}, 1 \mathrm{H}, J=7.7 \mathrm{~Hz}), 7.11(\mathrm{t}, 1 \mathrm{H}, J=7.7 \mathrm{~Hz}), 7.03(\mathrm{t}, 1 \mathrm{H}, J=7.7 \mathrm{~Hz}), 6.65(\mathrm{~s}, 2 \mathrm{H}), 6.33(\mathrm{~s}, 1 \mathrm{H}), 3.99(\mathrm{t}, 2 \mathrm{H}, J=6.4 \mathrm{~Hz})$, $3.79(\mathrm{~s}, 3 \mathrm{H}), 3.78(\mathrm{~s}, 6 \mathrm{H}), 3.26\left(\mathrm{t}, 2 \mathrm{H}, J=6.4 \mathrm{~Hz}\right.$ ), $2.92(\mathrm{~s}, 3 \mathrm{H}) ;{ }^{13} \mathrm{C}$ NMR (DMSO-d $\left., 100 \mathrm{MHz}, 363 \mathrm{~K}\right): \delta 168.50$, $153.58,140.27,137.93,136.83,135.78,130.69,129.04,123.67,122.69,119.13,118.60,111.64,110.88,107.91$, 60.57, 57.22, 47.89, 32.07, 24.16; HRMS(EI) calcd. for $\mathrm{C}_{23} \mathrm{H}_{24} \mathrm{~N}_{2} \mathrm{O}_{4}: \mathrm{m} / \mathrm{z} 392.1736$, found $\mathrm{m} / \mathrm{z} 392.1729$

3-Benzyl-1,2,3,7-tetrahydro-azocino[5,4-b]indol-4-one (2j). Synthesized from amide $11181 \mathrm{mg}$ with $15 \mathrm{mg}$ $\mathrm{Hg}(\mathrm{OTf})_{2}(5 \mathrm{~mol} \%)$. Conventional heating (oil bath) - $50 \stackrel{\circ}{\circ}$, $48 \mathrm{~h}$. Eluent DCM / $\mathrm{Et}_{2} \mathrm{O}(4 / 1)\left(R_{f} 0.31\right)$. Yield: $45 \mathrm{mg}$ (25\%). ${ }^{1} \mathrm{H}$ NMR (DMSO- $\left.d_{6}, 300 \mathrm{MHz}\right): \delta 11.04(\mathrm{~s}, 1 \mathrm{H}), 7.23-7.47(\mathrm{~m}, 7 \mathrm{H}), 7.13(\mathrm{t}, 1 \mathrm{H}, J=7.4 \mathrm{~Hz}), 6.987 .13(\mathrm{t}, 1 \mathrm{H}, J$ $=7.4 \mathrm{~Hz}), 5.92(\mathrm{~s}, 2 \mathrm{H}), 4.69(\mathrm{~s}, 2 \mathrm{H}), 3.64(\mathrm{~m}, 2 \mathrm{H}), 2.79(\mathrm{~m}, 2 \mathrm{H}) ;{ }^{13} \mathrm{C} \mathrm{NMR}\left(\mathrm{CDCl}_{3}, 75 \mathrm{MHz}\right): \delta 170.52,138.27$, 137.39, 136.38, 129.05, 128.76, 128.28, 128.18, 127.62, 123.17, 120.62, 119.68, 118.58, 113.11, 111.36, 50.60, 47.01, 24.48; HRMS(EI) calcd. for $\mathrm{C}_{20} \mathrm{H}_{18} \mathrm{~N}_{2} \mathrm{O}: \mathrm{m} / \mathrm{z} 302.1419$, found $\mathrm{m} / \mathrm{z} 302.1418$

3-Methyl-1,2,3,7-tetrahydro-azocino[5,4-b]indol-4-one (2k). Synthesized from amide $1 \mathrm{~m} 136 \mathrm{mg}$ with $15 \mathrm{mg}$ $\mathrm{Hg}(\mathrm{OTf})_{2}(5 \mathrm{~mol} \%)$. Conventional heating (oil bath) - $50 \stackrel{\circ}{\circ} \mathrm{C}, 48 \mathrm{~h}$. Eluent DCM / MeCN (3/1) $\left(R_{f} 0.14\right)$. Yield: $29 \mathrm{mg}$ (21\%). ${ }^{1} \mathrm{H}$ NMR (DMSO- $\left.d_{6}, 400 \mathrm{MHz}\right): \delta 10.98(\mathrm{~s}, 1 \mathrm{H}), 7.45(\mathrm{~d}, 1 \mathrm{H}, J=7.8 \mathrm{~Hz}), 7.32(\mathrm{~d}, 1 \mathrm{H}, J=7.8 \mathrm{~Hz}), 7.13(\mathrm{t}, 1 \mathrm{H}, J$ $=7.8 \mathrm{~Hz}), 7.00(\mathrm{~d}, 1 \mathrm{H}, J=7.8 \mathrm{~Hz}), 5.87(\mathrm{~s}, 1 \mathrm{H}), 5.82(\mathrm{~s}, 1 \mathrm{H}), 3.62-3.69(\mathrm{~m}, 2 \mathrm{H}), 3.04(\mathrm{~s}, 3 \mathrm{H}), 2.88-2.95(\mathrm{~m}, 2 \mathrm{H}) ;{ }^{13} \mathrm{C}$ NMR $\left(\mathrm{CDCl}_{3}, 100 \mathrm{MHz}\right): \delta 168.91,138.60,136.54,129.48,128.27,123.05,120.56,119.37,118.97,113.26,111.53$, 49.31, 34.77, 24.21; HRMS(EI) calcd. for $\mathrm{C}_{14} \mathrm{H}_{14} \mathrm{~N}_{2} \mathrm{O}: \mathrm{m} / \mathrm{z} 226.1106$, found $\mathrm{m} / \mathrm{z} 226.1105$ 


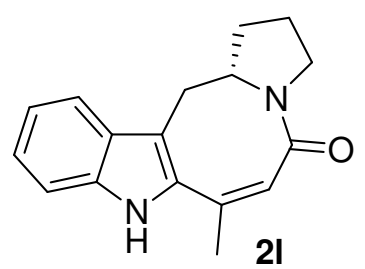

Indoloazocinone 21. Synthesized from amide $1 \mathrm{p} 160 \mathrm{mg}$ with $15 \mathrm{mg} \mathrm{Hg}(\mathrm{OTf})_{2}(5 \mathrm{~mol} \%)$. MW irradiation - $90 \mathrm{~W}$,

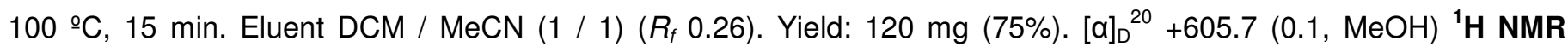
(DMSO-d $d_{6}, 400 \mathrm{MHz}$ ) : $\delta 10.82(\mathrm{~s}, 1 \mathrm{H}), 7.45(\mathrm{~d}, 1 \mathrm{H}, J=7.8 \mathrm{~Hz}), 7.30(\mathrm{~d}, 1 \mathrm{H}, J=7.8 \mathrm{~Hz}), 7.12(\mathrm{t}, 1 \mathrm{H}, J=7.8 \mathrm{~Hz}), 6.99$ (t, $1 \mathrm{H}, J=7.8 \mathrm{~Hz}), 5.92(\mathrm{~d}, 1 \mathrm{H}, J=1.2 \mathrm{~Hz}), 4.54-4.74(\mathrm{~m}, 1 \mathrm{H}), 3.46-3.56(\mathrm{~m}, 1 \mathrm{H}), 3.06-3.23(\mathrm{~m}, 2 \mathrm{H}), 2.83\left(\mathrm{dd}, 1 \mathrm{H}, J_{1}\right.$ $\left.=3.6 \mathrm{~Hz}, J_{2}=16.6 \mathrm{~Hz}\right), 2.24(\mathrm{~d}, 3 \mathrm{H}, J=1.2 \mathrm{~Hz}), 1.90-2.20(\mathrm{~m}, 3 \mathrm{H}), 1.79\left(\mathrm{dd}, 1 \mathrm{H}, J_{1}=6.9 \mathrm{~Hz}, J_{2}=11.7 \mathrm{~Hz}\right) ;{ }^{13} \mathbf{C ~ N M R}$ (DMSO-d $d_{6}, 100 \mathrm{MHz}, 363 \mathrm{~K}$ ) : $\delta$ 166.42, 136.50, 134.15, 132.57, 129.10, 124.67, 122.61, 119.14, 118.77, 111.30, 109.99, 56.65, 44.30, 32.11, 31.56, 24.04, 21.91; HRMS(EI) calcd. for $\mathrm{C}_{17} \mathrm{H}_{18} \mathrm{~N}_{2} \mathrm{O}: \mathrm{m} / \mathrm{z} 266.1419$, found m/z 266.1420

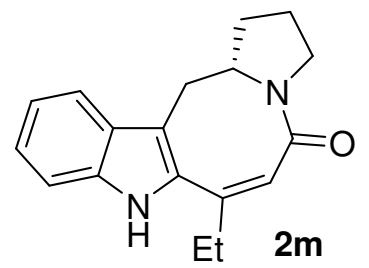

Indoloazocinone $2 \mathrm{~m}$. Synthesized from amide $1 \mathrm{q} 168 \mathrm{mg}$ with $15 \mathrm{mg} \mathrm{Hg}(\mathrm{OTf})_{2}$ (5 mol\%). MW irradiation - 90

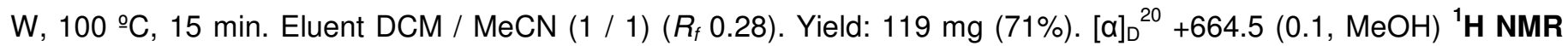
(DMSO- $\left.d_{6}, 400 \mathrm{MHz}\right): \delta 10.80(\mathrm{~s}, 1 \mathrm{H}), 7.44(\mathrm{~d}, 1 \mathrm{H}, J=7.7 \mathrm{~Hz}), 7.28(\mathrm{~d}, 1 \mathrm{H}, J=7.7 \mathrm{~Hz}), 7.10(\mathrm{t}, 1 \mathrm{H}, J=7.7 \mathrm{~Hz}), 6.98$ (t, $1 \mathrm{H}, J=7.7 \mathrm{~Hz}), 5.89(\mathrm{~s}, 1 \mathrm{H}), 4.64-4.72(\mathrm{~m}, 1 \mathrm{H}), 3.43-3.53(\mathrm{~m}, 1 \mathrm{H}), 3.13-3.20(\mathrm{~m}, 1 \mathrm{H}), 3.08\left(\mathrm{dd}, 1 \mathrm{H}, J_{1}=12.9 \mathrm{~Hz}\right.$, $\left.J_{2}=16.5 \mathrm{~Hz}\right), 2.82\left(\mathrm{dd}, 1 \mathrm{H}, J_{1}=3.8 \mathrm{~Hz}, J_{2}=16.5 \mathrm{~Hz}\right), 2.60-2.71(\mathrm{~m}, 1 \mathrm{H}), 2.50-2.59(\mathrm{~m}, 1 \mathrm{H}), 1.89-2.23(\mathrm{~m}, 3 \mathrm{H}), 1.78$ $\left(\mathrm{dd}, 1 \mathrm{H}, J_{1}=6.9 \mathrm{~Hz}, J_{2}=11.7 \mathrm{~Hz}\right), 1.00$ (t, 3H, $J=7.4 \mathrm{~Hz}$ ); ${ }^{13} \mathrm{C}$ NMR (DMSO-d, $100 \mathrm{MHz}$ ) : $\delta 166.25,139.81$, 135.67, 131.01, 128.38, 122.56, 122.11, 118.55, 118.34, 110.77, 110.04, 56.21, 44.00, 31.12, 30.79, 29.91, 21.25, 12.90; HRMS(EI) calcd. for $\mathrm{C}_{18} \mathrm{H}_{20} \mathrm{~N}_{2} \mathrm{O}: \mathrm{m} / \mathrm{z} 280.1576$, found m/z 266.1575

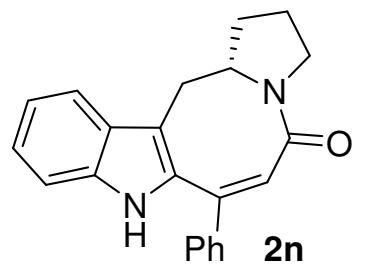

Indoloazocinone 2n. Synthesized from amide 1r $197 \mathrm{mg}$ with $75 \mathrm{mg} \mathrm{Hg}(\mathrm{OTf})_{2}$ (25 mol\%). MW irradiation - 90

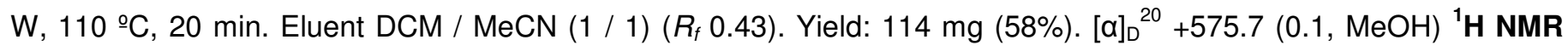
(DMSO- $\left.d_{6}, 400 \mathrm{MHz}, 363 \mathrm{~K}\right): \delta 10.18,7.49(\mathrm{~d}, 1 \mathrm{H}, J=7.8 \mathrm{~Hz}), 7.29-7.40(\mathrm{~m}, 5 \mathrm{H}), 7.22(\mathrm{~d}, 1 \mathrm{H}, J=7.8 \mathrm{~Hz}), 7.07(\mathrm{t}$, $1 \mathrm{H}, J=7.8 \mathrm{~Hz}), 6.98(\mathrm{t}, 1 \mathrm{H}, J=7.8 \mathrm{~Hz}), 6.31(\mathrm{~s}, 1 \mathrm{H}), 4.69-4.77(\mathrm{~m}, 1 \mathrm{H}), 3.52-3.61(\mathrm{~m}, 1 \mathrm{H}), 3.11-3.22(\mathrm{~m}, 2 \mathrm{H}), 3.00$ (dd, $\left.1 \mathrm{H}, J_{1}=4.4 \mathrm{~Hz}, J_{2}=16.4 \mathrm{~Hz}\right), 2.04-2.32(\mathrm{~m}, 2 \mathrm{H}), 1.89-2.00(\mathrm{~m}, 1 \mathrm{H}), 1.77-1.87(\mathrm{~m}, 1 \mathrm{H}) ;{ }^{13} \mathrm{C}$ NMR (DMSO- $d_{6}, 100$ $\mathrm{MHz}, 363 \mathrm{~K}): \delta 166.59,140.60,138.22,136.73,130.74,128.80,128.77,128.20,125.50,122.64,119.09,118.71$, 112.29, 111.62, 56.99, 44.48, 31.62, 31.60, 21.88; HRMS(EI) calcd. for $\mathrm{C}_{22} \mathrm{H}_{20} \mathrm{~N}_{2} \mathrm{O}: \mathrm{m} / \mathrm{z} 328.1576$, found $\mathrm{m} / \mathrm{z}$ 328.1597 
Synthesized from amide $1 \mathrm{u} 302 \mathrm{mg}$ with $30 \mathrm{mg} \mathrm{Hg}(\mathrm{OTf})_{2}$ (10 mol\%). MW irradiation - $90 \mathrm{~W}, 100 \stackrel{\circ}{\circ}, 25 \mathrm{~min}$. Eluent $\mathrm{DCM} / \mathrm{Et}_{2} \mathrm{O}(4 / 1)\left(R_{f} 0.28\right)$. Yield: $236 \mathrm{mg}(78 \%)$. [a $]_{\mathrm{D}}{ }^{20}-152.7\left(1.0, \mathrm{CHCl}_{3}\right)^{1} \mathrm{H}$ NMR (DMSO- $\left.d_{6}, 400 \mathrm{MHz}\right): \delta 10.87$ $(\mathrm{s}, 1 \mathrm{H}), 7.32(\mathrm{~d}, 1 \mathrm{H}, J=7.8 \mathrm{~Hz}), 7.25(\mathrm{~d}, 1 \mathrm{H}, J=7.8 \mathrm{~Hz}), 7.03-7.17(\mathrm{~m}, 6 \mathrm{H}), 6.98(\mathrm{t}, 1 \mathrm{H}, J=7.8 \mathrm{~Hz}), 6.06(\mathrm{~d}, 1 \mathrm{H}, J=$ $1.2 \mathrm{~Hz}), 4.92-5.03(\mathrm{~m}, 1 \mathrm{H}), 4.58(\mathrm{~d}, 1 \mathrm{H}, J=15.7 \mathrm{~Hz}), 4.52(\mathrm{~d}, 1 \mathrm{H}, J=15.7 \mathrm{~Hz}), 3.81(\mathrm{~d}, 2 \mathrm{H}, J=6.1 \mathrm{~Hz}), 3.10(\mathrm{dd}, 1 \mathrm{H}$, $\left.J_{1}=13.0 \mathrm{~Hz}, J_{2}=16.7 \mathrm{~Hz}\right), 2.91\left(\mathrm{dd}, 1 \mathrm{H}, J_{1}=3.0 \mathrm{~Hz}, J_{2}=16.7 \mathrm{~Hz}\right), 2.27(\mathrm{~d}, 3 \mathrm{H}, J=1.2 \mathrm{~Hz}), 0.90-1.08(\mathrm{~m}, 21 \mathrm{H}) ;{ }^{13} \mathrm{C}$ NMR $\left(\mathrm{CDCl}_{3}, 100 \mathrm{MHz}\right): \delta 717.02,138.30,136.29,135.77,131.96,128.36,128.27,127.18,126.82,122.55,121.70$, 118.88, 118.34, 111.34, 108.73, 64.72, 59.11, 44.89, 27.01, 24.13, 18.07, 12.05; LRMS(CI) (m/z, relative intensity) : $503\left([\mathrm{M}+\mathrm{H}]^{+}, 100\right), 459(23)$

(S)-3-Benzyl-6-pentyl-2-triisopropylsilanyloxymethyl-1,2,3,7-tetrahydro-azocino[5,4-b]indol-4-one (2p). Synthesized from amide 1v $335 \mathrm{mg}$ with $60 \mathrm{mg} \mathrm{Hg}(\mathrm{OTf})_{2}$ (20 mol\%). MW irradiation - $90 \mathrm{~W}, 100 \stackrel{\circ}{\circ}, 45 \mathrm{~min}$. Eluent $\mathrm{DCM} / \mathrm{Et}_{2} \mathrm{O}(20 / 1)\left(R_{f} 0.12\right)$. Yield: $238 \mathrm{mg}(71 \%) .[\alpha]_{\mathrm{D}}{ }^{20}-105.1\left(1.0, \mathrm{CHCl}_{3}\right){ }^{1} \mathrm{H}$ NMR (DMSO- $\left.d_{6}, 400 \mathrm{MHz}\right): \delta 10.87$ $(\mathrm{s}, 1 \mathrm{H}), 7.31(\mathrm{~d}, 1 \mathrm{H}, J=7.9 \mathrm{~Hz}), 7.26(\mathrm{~d}, 1 \mathrm{H}, J=7.9 \mathrm{~Hz}), 7.04-7.15(\mathrm{~m}, 6 \mathrm{H}), 6.98(\mathrm{t}, 1 \mathrm{H}, J=7.9 \mathrm{~Hz}), 6.05(\mathrm{~s}, 1 \mathrm{H})$, 4.93-5.04 (m, 1H), $4.53(\mathrm{~d}, 1 \mathrm{H}, J=16.3 \mathrm{~Hz}), 4.49(\mathrm{~d}, 1 \mathrm{H}, J=16.3 \mathrm{~Hz}), 3.77(\mathrm{~d}, 2 \mathrm{H}, J=6.4 \mathrm{~Hz}), 3.09\left(\mathrm{dd}, 1 \mathrm{H}, J_{1}=\right.$ $\left.13.1 \mathrm{~Hz}, J_{2}=16.5 \mathrm{~Hz}\right), 2.80-2.94(\mathrm{~m}, 2 \mathrm{H}), 2.40-2.52(\mathrm{~m}, 1 \mathrm{H}), 1.16-1.42(\mathrm{~m}, 6 \mathrm{H}), 0.89-1.04(\mathrm{~m}, 21 \mathrm{H}), 0.79(\mathrm{t}, 3 \mathrm{H}, J=$ $6.6 \mathrm{~Hz}) ;{ }^{13} \mathrm{C}$ NMR $\left(\mathrm{CDCl}_{3}, 100 \mathrm{MHz}\right): \delta 171.13,140.28,138.30,136.17,131.00,128.38,128.25,127.16,127.77$, $122.37,121.33,118.72,118.20,111.24,109.45,64.61,59.33,44.61,37.89,31.39,27.69,26.87,22.42,18.02,14.00$, 11.97; LRMS(Cl) (m/z, relative intensity) : $559\left([\mathrm{M}+\mathrm{H}]^{+}, 100\right), 516(28), 371(10)$.

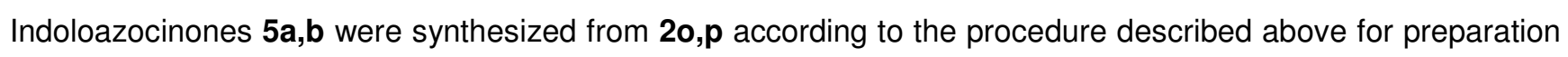
of $11, m$ from $12 a, b$ :

(S)-3-Benzyl-2-hydroxymethyl-6-methyl-1,2,3,7-tetrahydro-azocino[5,4-b]indol-4-one (5a). Synthesized from $20504 \mathrm{mg}$ (1 mmol). Yield: $297 \mathrm{mg}(86 \%)$. [a] ${ }^{20}{ }^{20}-266.4\left(1.0, \mathrm{CHCl}_{3}\right)^{1} \mathrm{H}$ NMR (DMSO-d $\left.6,400 \mathrm{MHz}\right): \delta 10.85$ (s, $1 \mathrm{H}), 7.31(\mathrm{~d}, 1 \mathrm{H}, J=8.0 \mathrm{~Hz}), 7.17(\mathrm{~d}, 1 \mathrm{H}, J=8.0 \mathrm{~Hz}), 7.05-7.14(\mathrm{~m}, 4 \mathrm{H}), 6.97-7.03(\mathrm{~m}, 2 \mathrm{H}), 6.95(\mathrm{t}, 1 \mathrm{H}, J=8.0 \mathrm{~Hz})$, $6.06(\mathrm{~d}, 1 \mathrm{H}, J=1.1 \mathrm{~Hz}), 4.86-4.96(\mathrm{~m}, 2 \mathrm{H}), 4.69(\mathrm{~d}, 1 \mathrm{H}, J=15.7 \mathrm{~Hz}), 4.40(\mathrm{~d}, 1 \mathrm{H}, J=15.7 \mathrm{~Hz}), 3.51-3.63(\mathrm{~m}, 2 \mathrm{H})$, 2.81-2.93 (m, 2H), $2.27\left(\mathrm{~d}, 3 \mathrm{H}, J=1.1 \mathrm{~Hz}\right.$ ); ${ }^{13} \mathrm{C}$ NMR (DMSO- $\left.d_{6}, 100 \mathrm{MHz}\right): \delta 169.60,139.53,136.21,134.07$, $132.47,128.78,128.25,127.37,126.73,123.20,122.66,119.04,118.77,111.23,108.81,62.57,58.38,43.91,27.08$, 23.90; HRMS(EI) calcd. for $\mathrm{C}_{22} \mathrm{H}_{22} \mathrm{~N}_{2} \mathrm{O}_{2}: \mathrm{m} / \mathrm{z} 346.1681$, found $\mathrm{m} / \mathrm{z} 346.1676$

(S)-3-Benzyl-2-hydroxymethyl-6-pentyl-1,2,3,7-tetrahydro-azocino[5,4-b]indol-4-one (5b). Synthesized from 2p $446 \mathrm{mg}(0.8 \mathrm{mmol})$. Yield: $269 \mathrm{mg}(84 \%)$. $[\alpha]_{\mathrm{D}}{ }^{20}-158.4\left(1.0, \mathrm{CHCl}_{3}\right)^{1} \mathbf{H} \mathbf{N M R}\left(\mathrm{CDCl}_{3}, 400 \mathrm{MHz}\right): \delta 9.33(\mathrm{~s}$, $1 \mathrm{H}), 7.19-7.30(\mathrm{~m}, 5 \mathrm{H}), 7.10-7.18(\mathrm{~m}, 3 \mathrm{H}), 7.04(\mathrm{t}, 1 \mathrm{H}, J=7.5 \mathrm{~Hz}), 6.11(\mathrm{~s}, 1 \mathrm{H}), 5.09-5.23(\mathrm{~m}, 1 \mathrm{H}), 4.81(\mathrm{~d}, 1 \mathrm{H}, J=$ $15.4 \mathrm{~Hz}), 4.70(\mathrm{~d}, 1 \mathrm{H}, J=15.4 \mathrm{~Hz}), 3.82-3.91(\mathrm{~m}, 1 \mathrm{H}), 3.69-3.78(\mathrm{~m}, 1 \mathrm{H}), 2.94\left(\mathrm{dd}, 1 \mathrm{H}, J_{1}=13.1 \mathrm{~Hz}, J_{2}=16.4 \mathrm{~Hz}\right)$, 2.73-2.86 (m, 2H), 2.45-2.54 (m, $1 \mathrm{H}), 2.36(\mathrm{t}, 1 \mathrm{H}, J=5.8 \mathrm{~Hz}), 1.23-1.52(\mathrm{~m}, 6 \mathrm{H}), 0.88(\mathrm{t}, 3 \mathrm{H}, J=7.0 \mathrm{~Hz}) ;{ }^{13} \mathrm{C} \mathrm{NMR}$ $\left(\mathrm{CDCl}_{3}, 100 \mathrm{MHz}\right): \delta 171.81,140.33,138.41,136.18,131.29,128.79,128.46,127.36,122.66,121.26,119.08$, 118.29, 111.21, 108.98, 63.21, 59.33, 44.35, 37.81, 31.61, 27.77, 26.22, 22.43, 14.10; HRMS(EI) calcd. for $\mathrm{C}_{26} \mathrm{H}_{30} \mathrm{~N}_{2} \mathrm{O}_{2}: \mathrm{m} / \mathrm{z}$ 402.2307, found $\mathrm{m} / \mathrm{z} 402.2292$ 


\section{General procedure for hydrogenation of $2 \mathrm{a}, \mathrm{d}, \mathrm{j}$ and $5 \mathrm{a}$ :}

Indoloazocinone $2(0.2 \mathrm{mmol})$ was dissolved in an appropriate degassed solvent. 10\% Pd/C $32 \mathrm{mg}(15 \mathrm{~mol} \%)$ was added and the resulting suspension was evacuated and flushed back with argon three times. The reaction flask was purged with $\mathrm{H}_{2}$ from a balloon and maintained with stirring at $\mathrm{rt}$ for $24 \mathrm{~h}$. The catalyst was filtered off and the filtrate was evaporated with silica. Pure indoloazocinones $6 \mathbf{a - d}$ were isolated by column chromatography on silica gel.

3-Benzyl-6-methyl-1,2,3,5,6,7-hexahydro-azocino[5,4-b]indol-4-one (6a). Synthesized from 2a $63 \mathrm{mg}$. Solvent - EtOH / THF 5:2. Eluent DCM / EtOAc (1 / 1) $\left(R_{f} 0.40\right)$. Yield: $60 \mathrm{mg}(95 \%)$. ${ }^{1} \mathrm{H}$ NMR (DMSO- $\left.d_{6}, 400 \mathrm{MHz}\right)$ : $\delta 10.73(\mathrm{~s}, 1 \mathrm{H}), 7.32(\mathrm{~d}, 1 \mathrm{H}, J=8.0 \mathrm{~Hz}), 7.26(\mathrm{~d}, 1 \mathrm{H}, J=8.0 \mathrm{~Hz}), 7.06-7.14(\mathrm{~m}, 3 \mathrm{H}), 6.97-7.03(\mathrm{~m}, 3 \mathrm{H}), 6.91(\mathrm{t}, 1 \mathrm{H}, J$ $=7.5 \mathrm{~Hz}), 4.33(\mathrm{~d}, 1 \mathrm{H}, J=15.3 \mathrm{~Hz}), 4.16(\mathrm{~d}, 1 \mathrm{H}, J=15.3 \mathrm{~Hz}), 3.68-3.75(\mathrm{~m}, 2 \mathrm{H}), 3.52-3.61(\mathrm{~m}, 1 \mathrm{H}), 2.98-3.16(\mathrm{~m}$, 2H), $2.90\left(\mathrm{dd}, 1 \mathrm{H}, J_{1}=7.1 \mathrm{~Hz}, J_{2}=13.5 \mathrm{~Hz}\right), 2.79\left(\mathrm{dd}, 1 \mathrm{H}, J_{1}=10.5 \mathrm{~Hz}, J_{2}=13.5 \mathrm{~Hz}\right), 1.37(\mathrm{~d}, 3 \mathrm{H}, J=7.0 \mathrm{~Hz}) ;{ }^{13} \mathrm{C}$ NMR $\left(\mathrm{CDCl}_{3}, 100 \mathrm{MHz}\right): \delta 173.68,138.14,137.03,135.21,129.35,128.32,127.81,127.11,121.08,118.99,117.18$, 110.70, 105.09, 50.13, 46.25, 41.73, 31.96, 22.85, 21.68; HRMS(EI) calcd. for $\mathrm{C}_{21} \mathrm{H}_{22} \mathrm{~N}_{2} \mathrm{O}: \mathrm{m} / \mathrm{z} 318.1732$, found m/z 318.1726

3,6-Dimethyl-1,2,3,5,6,7-hexahydro-azocino[5,4-b]indol-4-one (6b). Synthesized from 2d $48 \mathrm{mg}$. Solvent $\mathrm{EtOH} / \mathrm{THF}$ 5:2. Eluent DCM / MeCN (1 / 1) ( $\left.R_{f} 0.31\right)$. Yield: $47 \mathrm{mg}(98 \%) .{ }^{1} \mathbf{H}$ NMR (DMSO- $\left.d_{6}, 400 \mathrm{MHz}\right): \delta 10.69(\mathrm{~s}$, $1 \mathrm{H}), 7.39(\mathrm{~d}, 1 \mathrm{H}, J=7.7 \mathrm{~Hz}), 7.24(\mathrm{~d}, 1 \mathrm{H}, J=7.7 \mathrm{~Hz}), 6.99(\mathrm{t}, 1 \mathrm{H}, J=7.7 \mathrm{~Hz}), 6.93(\mathrm{t}, 1 \mathrm{H}, J=7.7 \mathrm{~Hz}), 3.87-3.96(\mathrm{~m}$, $1 \mathrm{H})$, 3.69-3.77 $(\mathrm{m}, 1 \mathrm{H}), 3.73\left(\mathrm{dt}, 1 \mathrm{H}, J_{1}=5.4 \mathrm{~Hz}, J_{2}=15.1 \mathrm{~Hz}\right), 2.97-3.14(\mathrm{~m}, 2 \mathrm{H}), 2.92\left(\mathrm{dd}, 1 \mathrm{H}, J_{1}=7.8 \mathrm{~Hz}, J_{2}=13.8\right.$ $\mathrm{Hz}$ ), 2.54-2.63 (m, 4H), 1.36 (d, 3H, $J=6.9 \mathrm{~Hz}$ ); ${ }^{13} \mathrm{C}$ NMR (DMSO- $d_{6}, 100 \mathrm{MHz}$ ) : $\delta 172.29,138.67,135.40,129.64$, 120.66, 118.66, 117.47, 111.06, 104.95, 48.17, 42.67, 33.65, 30.66, 22.65, 21.32; HRMS(EI) calcd. for $\mathrm{C}_{15} \mathrm{H}_{18} \mathrm{~N}_{2} \mathrm{O}$ : $\mathrm{m} / \mathrm{z} 242.1419$, found $\mathrm{m} / \mathrm{z} 242.1402$

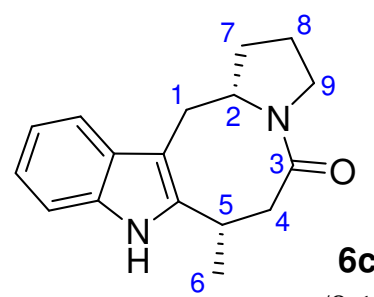

$\alpha / \beta$ 1:20 Partial assignment for $6 \mathbf{c}$ ( $\beta$-epimer) is based on COSY and HMQC spectra. Configuration at $\mathrm{C} 5$ is confirmed by NOE effect between $\mathrm{H}-\mathrm{C} 2$ and $\mathrm{H}-\mathrm{C} 5$.

Indoloazocinone 6c. Synthesized from 2j $54 \mathrm{mg}$. Solvent - EtOH. Eluent DCM / MeCN (1/1) $\left(R_{f} 0.17\right)$. Yield: $50 \mathrm{mg}(94 \%)$. (the major $\beta$-epimer is described) ${ }^{1} \mathrm{H}$ NMR (DMSO- $\left.d_{6}, 400 \mathrm{MHz}\right): \delta 10.68(\mathrm{~s}, 1 \mathrm{H}), 7.41(\mathrm{~d}, 1 \mathrm{H}, J=7.6$ $\mathrm{Hz}$ ), $7.28(\mathrm{~d}, 1 \mathrm{H}, J=7.6 \mathrm{~Hz}$ ), 7.00 (t, $1 \mathrm{H}, J=7.6 \mathrm{~Hz}), 6.94(\mathrm{t}, 1 \mathrm{H}, J=7.6 \mathrm{~Hz}), 4.32-4.40(\mathrm{~m}, \mathrm{H}-\mathrm{C} 2), 3.54-3.64(\mathrm{~m}, \mathrm{H}-$ C5), 3.27-3.37 (m, H-C4, H-C9), 3.13 (dd, H-C1, J1 = 5.6 Hz, J2 =15.6 Hz), 2.84-2.96 (m, H-C1, H-C9), 2.24 (dd, H$\left.\mathrm{C} 4, J_{1}=6.5 \mathrm{~Hz}, J_{2}=13.9 \mathrm{~Hz}\right), 2.07-2.18(\mathrm{~m}, \mathrm{H}-\mathrm{C} 7), 1.64-1.84(\mathrm{~m}, \mathrm{H}-\mathrm{C} 7,2 \mathrm{H}-\mathrm{C} 8), 1.43(\mathrm{~d}, 3 \mathrm{H}-\mathrm{C} 6, \mathrm{~J}=7.1 \mathrm{~Hz}) ;{ }^{13} \mathrm{C}$ NMR (DMSO- $\left.d_{6}, 100 \mathrm{MHz}\right): \delta 169.58$ (C3), 139.41, 135.33, 129.63, 120.46, 118.79, 117.48, 111.25, 105.84, 57.31 (C2), 46.63 (C9), 43.08 (C4), 34.27 (C7), 29.92 (C5), 29.69 (C1), 21.61 (C8), 19.49 (C6); HRMS(EI) calcd. for $\mathrm{C}_{17} \mathrm{H}_{20} \mathrm{~N}_{2} \mathrm{O}: \mathrm{m} / \mathrm{z} 268.1576$, found $\mathrm{m} / \mathrm{z} 268.1565$ 


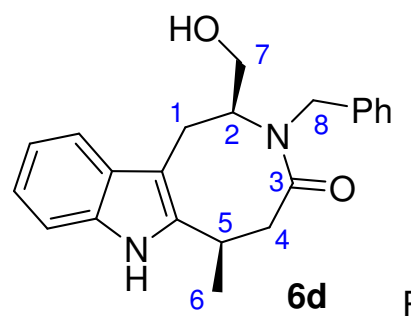

Partial assignment for $\mathbf{6} \mathbf{d}$ is based on COSY and HMQC spectra.

(2S,6R)-3-Benzyl-2-hydroxymethyl-6-methyl-1,2,3,5,6,7-hexahydro-azocino[5,4-b]indol-4-one

$(6 d)$.

Synthesized from 5a $70 \mathrm{mg}$. Solvent - EtOH. Eluent DCM / MeCN (1 / 1) $\left(R_{f} 0.25\right)$. Yield: $67 \mathrm{mg}(96 \%)$. [a $]_{D}{ }^{20}-136.3$ $(1.0, \mathrm{MeOH}){ }^{1} \mathrm{H}$ NMR (DMSO- $\left.d_{6}, 400 \mathrm{MHz}, 363 \mathrm{~K}\right): \delta 10.46(\mathrm{~s}, 1 \mathrm{H}), 7.25(\mathrm{~d}, 1 \mathrm{H}, J=7.9 \mathrm{~Hz}), 7.13(\mathrm{~d}, 1 \mathrm{H}, J=7.9 \mathrm{~Hz})$, $6.97(\mathrm{t}, 1 \mathrm{H}, J=7.9 \mathrm{~Hz}), 6.80(\mathrm{t}, 1 \mathrm{H}, J=7.9 \mathrm{~Hz}), 6.73(\mathrm{t}, 1 \mathrm{H}, J=7.3 \mathrm{~Hz}), 6.50(\mathrm{t}, 2 \mathrm{H}, J=7.3 \mathrm{~Hz}), 6.35(\mathrm{~d}, 2 \mathrm{H}, J=7.3$ $\mathrm{Hz}$ ), 4.95 (d, H-C8, J=15.4 Hz), 4.69 (t, H-O, J = 5.2 Hz), 3.86 (d, H-C8, $J=15.4 \mathrm{~Hz}), 3.68-3.79(\mathrm{~m}, 2 \mathrm{H}-\mathrm{C} 7)$, 3.513.61 (m, H-C2), 3.36-3.47 (m, H-C5), 3.19-3.35 (m, H-C4, H-C1), 3.12 (dd, H-C1, J = 7.8 Hz, J = 15.2 Hz), 2.49 (dd, $\mathrm{H}-\mathrm{C} 4, J_{1}=5.4 \mathrm{~Hz}, J_{2}=12.4 \mathrm{~Hz}$ ), $1.34\left(\mathrm{~d}, 3 \mathrm{H}-\mathrm{C} 6, J=6.7 \mathrm{~Hz}\right.$ ); ${ }^{13} \mathrm{C}$ NMR (DMSO-d $\left., 100 \mathrm{MHz}, 363 \mathrm{~K}\right): \delta 172.96(\mathrm{C} 3)$, 140.22, 138.15, 136.03, 129.63, 127.65, 127.04, 126.08, 120.50, 118.60, 117.26, 110.82, 105.53, 64.42 (C7), 61.11 (C2), 51.19 (C8), 42.52 (C4), 33.00 (C5), 26.29 (C1), 22.68 (C6); HRMS(EI) calcd. for $\mathrm{C}_{22} \mathrm{H}_{24} \mathrm{~N}_{2} \mathrm{O}_{2}: \mathrm{m} / \mathrm{z} 348.1838$, found $\mathrm{m} / \mathrm{z} 348.1828$

\section{General procedure for $\mathrm{LAH}$ reduction of $2 \mathrm{a}, \mathbf{e , g}$ :}

Indoloazocinone $2(0.5 \mathrm{mmol})$ was dissolved under argon in dry THF $(5 \mathrm{~mL})$. To the resulting solution LAH powder $158 \mathrm{mg}$ (5 equiv) was added in one portion. The reaction mixture was kept with stirring at $60{ }^{\circ} \mathrm{C}$ for approximately $12 \mathrm{~h}$. After cooling down to rt $15 \%$ aqueous $\mathrm{NaOH}$ was added dropwise until a solid granular precipitate was formed. The precipitate was excessively washed with EtOAc. The EtOAc layer was washed with brine, dried $\left(\mathrm{Na}_{2} \mathrm{SO}_{4}\right)$ and evaporated with silica. Pure indoloazocines 7a-c were isolated by column chromatography on silica gel.

3-Benzyl-6-methyl-2,3,4,7-tetrahydro-1H-azocino[5,4-b]indole (7a). Synthesized from indoloazocinone 2a 158 mg. Eluent DCM / MeOH / $\mathrm{NH}_{3}$ (aq) (100/ $\left.10 / 0.1\right)\left(R_{f} 0.42\right)$. Yield: 144 mg (95\%). ${ }^{1} \mathrm{H}$ NMR (DMSO- $d_{6}, 400 \mathrm{MHz}$, $363 \mathrm{~K}): \delta 10.43(\mathrm{~s}, 1 \mathrm{H}), 7.45(\mathrm{~d}, 1 \mathrm{H}, J=7.9 \mathrm{~Hz}), 7.28-7.38(\mathrm{~m}, 5 \mathrm{H}), 7.21-7.26(\mathrm{~m}, 1 \mathrm{H}), 7.09(\mathrm{t}, 1 \mathrm{H}, J=7.3 \mathrm{~Hz}), 6.99$ (t, $1 \mathrm{H}, J=7.5 \mathrm{~Hz}), 5.72\left(\mathrm{dt}, 1 \mathrm{H}, J_{1}=1.5 \mathrm{~Hz}, J_{2}=7.8 \mathrm{~Hz}\right.$ ), $3.71(\mathrm{~s}, 2 \mathrm{H}), 3.17(\mathrm{~d}, 2 \mathrm{H}, J=8.0 \mathrm{~Hz}), 2.87-2.96(\mathrm{~m}, 4 \mathrm{H})$, $2.20\left(\mathrm{~d}, 3 \mathrm{H}, J=1.5 \mathrm{~Hz}\right.$ ); ${ }^{13} \mathrm{C}$ NMR (DMSO- $\left.d_{6}, 100 \mathrm{MHz}, 363 \mathrm{~K}\right): \delta 140.33,136.85,134.44,132.28,128.95,128.61$, 128.46, 127.04, 124.58, 121.76, 118.86, 118.40, 112.93, 111.27, 60.07, 51.37, 50.72, 23.25, 22.56; HRMS(EI) calcd. for $\mathrm{C}_{21} \mathrm{H}_{22} \mathrm{~N}_{2}: \mathrm{m} / \mathrm{z} 302.1783$, found $\mathrm{m} / \mathrm{z} 302.1780$

6-Ethyl-3-methyl-2,3,4,7-tetrahydro-1H-azocino[5,4-b]indole (7b). Synthesized from indoloazocinone 2e 127 mg. Eluent DCM / MeOH / NH 3 (aq) (150/ $15 / 1)\left(R_{f} 0.14\right)$. Yield: 116 mg (97\%). ${ }^{1} \mathbf{H}$ NMR (DMSO- $d_{6}, 400 \mathrm{MHz}, 363$ K) : $\delta 10.38(\mathrm{~s}, 1 \mathrm{H}), 7.45(\mathrm{~d}, 1 \mathrm{H}, J=7.8 \mathrm{~Hz}), 7.33(\mathrm{~d}, 1 \mathrm{H}, J=7.8 \mathrm{~Hz}), 7.08(\mathrm{t}, 1 \mathrm{H}, J=7.8 \mathrm{~Hz}), 6.99(\mathrm{t}, 1 \mathrm{H}, J=7.8 \mathrm{~Hz})$, $5.77(\mathrm{t}, 1 \mathrm{H}, J=7.8 \mathrm{~Hz}), 3.07(\mathrm{~d}, 2 \mathrm{H}, J=7.8 \mathrm{~Hz}), 2.84-2.90(\mathrm{~m}, 2 \mathrm{H}), 2.76-2.82(\mathrm{~m}, 2 \mathrm{H}), 2.56(\mathrm{q}, 2 \mathrm{H}, J=7.4 \mathrm{~Hz}), 2.36$ $(\mathrm{s}, 3 \mathrm{H}), 1.04\left(\mathrm{t}, 3 \mathrm{H}, J=7.4 \mathrm{~Hz}\right.$ ); ${ }^{13} \mathrm{C}$ NMR (DMSO- $\left.d_{6}, 100 \mathrm{MHz}, 363 \mathrm{~K}\right): \delta 138.53,136.94,133.58,128.52,123.04$, 121.66, 118.76, 118.27, 113.88, 111.28, 53.25, 52.76, 44.07, 29.10, 22.73, 13.74; HRMS(EI) calcd. for $\mathrm{C}_{16} \mathrm{H}_{20} \mathrm{~N}_{2}: \mathrm{m} / \mathrm{z}$ 240.1626 , found $\mathrm{m} / \mathrm{z} 240.1622$ 
6-Isopropyl-3-methyl-2,3,4,7-tetrahydro-1H-azocino[5,4-b]indole (7c). Synthesized from indoloazocinone $\mathbf{2 g}$ 134 mg. Eluent DCM / MeOH / $\mathrm{NH}_{3}(\mathrm{aq})(150 / 15 / 1)\left(R_{f} 0.35\right)$. Yield: $121 \mathrm{mg}(95 \%)$. ${ }^{1} \mathrm{H}$ NMR (DMSO- $d_{6}, 400 \mathrm{MHz}$, $363 \mathrm{~K}): \delta 10.37(\mathrm{~s}, 1 \mathrm{H}), 7.45(\mathrm{~d}, 1 \mathrm{H}, J=7.8 \mathrm{~Hz}), 7.34(\mathrm{~d}, 1 \mathrm{H}, J=7.8 \mathrm{~Hz}), 7.08(\mathrm{t}, 1 \mathrm{H}, J=7.8 \mathrm{~Hz}), 6.99(\mathrm{t}, 1 \mathrm{H}, J=7.8$ $\mathrm{Hz}$ ), $5.79\left(\mathrm{dt}, 1 \mathrm{H}, J_{1}=1.2 \mathrm{~Hz}, J_{2}=8.0 \mathrm{~Hz}\right.$ ), $3.01(\mathrm{~d}, 2 \mathrm{H}, J=8.0 \mathrm{~Hz}$ ), 2.91 (septet, $1 \mathrm{H}, J=6.7 \mathrm{~Hz}$ ), 2.80-2.85 (m, 2H), 2.70-2.75 (m, 2H), $2.36(\mathrm{~s}, 3 \mathrm{H}), 1.11(\mathrm{~d}, 6 \mathrm{H}, J=6.7 \mathrm{~Hz}) ;{ }^{13} \mathrm{C}$ NMR (DMSO- $\left.d_{6}, 100 \mathrm{MHz}, 363 \mathrm{~K}\right): \delta 142.85,137.01$, 134.05, 128.27, 121.78, 121.56, 118.73, 118.20, 114.19, 111.35, 53.42, 53.03, 44.15, 32.85, 22.75, 22.35; HRMS(EI) calcd. for $\mathrm{C}_{17} \mathrm{H}_{22} \mathrm{~N}_{2}: \mathrm{m} / \mathrm{z} 254.1783$, found $\mathrm{m} / \mathrm{z} 254.1778$

\section{Crystallographic data for $6 \mathrm{~d}$ :}

Crystals of $\mathbf{6 d}$, suitable for X-ray diffraction were obtained by slow evaporation from $\mathrm{MeCN}$ at rt. A transparent, rod-shaped crystal with approximate dimensions of $0.3 \times 0.15 \times 0.1 \mathrm{~mm}$, was selected and mounted in a nylon loop for data collection. X-ray intensity data were collected at $100 \mathrm{~K}$ on a SMART 6000 diffractometer equipped with CCD detector using CuKa radiation $(\lambda=1.54178 \AA)$, using $\varphi$ and $\omega$ scans. The images were interpreted and integrated with the program SAINT from Bruker [4], giving a total of 25331 reflections (3343 independent reflections, $\left.R_{\text {int }}=0.0695\right)$. The structure was solved by direct methods and refined by full-matrix least-squares on $\mathrm{F}^{2}$ using the SHELXTL program package [5], converging to a final $R=0.0507$ for 3059 reflections with $I>2 \sigma(I)$ and $\omega R 2=0.1445$ for all data and GOOF $=1.140$. Non-hydrogen atoms were anisotropically refined and the hydrogen atoms in the riding mode and isotropic temperature factors fixed at 1.2 times $\mathrm{U}(\mathrm{eq})$ of the parent atoms and 1.5 times $\mathrm{U}(\mathrm{eq})$ for a methyl and hydroxyl group. The absolute configuration of the structure was established by anomalous-dispersion effects.

Table 1. Summarized crystallographic data for $6 \mathrm{~d}$.

\begin{tabular}{|c|c|c|c|}
\hline formula & $\mathrm{C}_{22} \mathrm{H}_{24} \mathrm{~N}_{2} \mathrm{O}_{2}$ & $\omega R_{2}$ (all data) & 0.1445 \\
\hline$M\left(\mathrm{gmol}^{-1}\right)$ & 348.43 & GOOF & 1.140 \\
\hline crystal dimensions $\left(\mathrm{mm}^{3}\right)$ & $0.3 \times 0.15 \times 0.1$ & $\mu\left(\mathrm{mm}^{-1}\right)$ & 0.661 \\
\hline crystal system & orthorhombic & CCDC-entry & CCDC-735454 \\
\hline space group & $P 2_{1} 2_{1} 2_{1}$ & & \\
\hline$a(\AA)$ & $7.2291(3)$ & & \\
\hline$b(\AA)$ & $12.9611(5)$ & & \\
\hline$c(\AA)$ & $19.0394(6)$ & & \\
\hline$\alpha=\beta=\gamma\left({ }^{\circ}\right)$ & 90.00 & & \\
\hline$V\left(\AA^{3}\right)$ & $1783.94(12)$ & & \\
\hline Z & 4 & & \\
\hline$\rho_{\text {calc }}\left(\mathrm{gcm}^{-3}\right)$ & 1.297 & & \\
\hline $2 \theta_{\max }\left({ }^{\circ}\right)$ & 141.92 & & \\
\hline radiation & CuKa & & \\
\hline$\lambda(\AA)$ & 1.54178 & & \\
\hline$F(000)$ & 744 & & \\
\hline$T(\mathrm{~K})$ & $100(2)$ & & \\
\hline measured reflections & 25331 & & \\
\hline unique reflections & 3343 & & \\
\hline observed reflections $\left(\mathrm{I}_{0}>2 \sigma\left(\mathrm{I}_{0}\right)\right)$ & 3059 & & \\
\hline parameters refined & 237 & & \\
\hline$R_{1}$ & 0.0507 & & \\
\hline$\omega R_{2}{ }^{a}$ & 0.1384 & & \\
\hline$R_{1}$ (all data) & 0.0555 & & \\
\hline
\end{tabular}


Figure 1. Single crystal X-ray molecular structure of $6 \mathrm{~d}$, with atom-labeling scheme. Displacement ellipsoids are drawn at the $50 \%$ probability level.

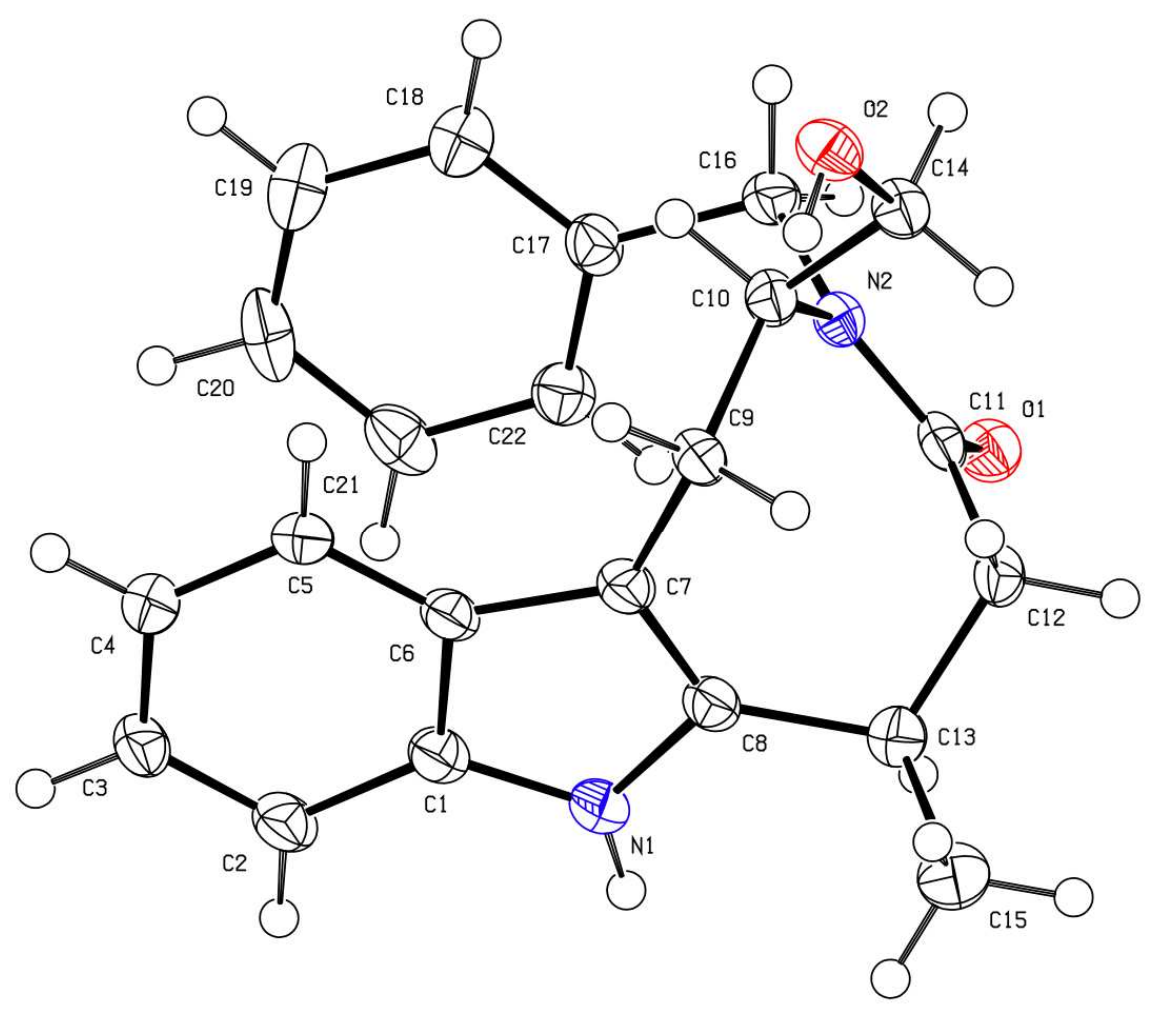

CCDC - 735454 contains the supplementary crystallographic data for this paper. These data can be obtained free of charge via www.cam.ac.uk/conts/retrieving.html (or from the Cambridge Crystallographic Data Centre, 12 Union Road, Cambridge CB2 1EZ, UK; fax: (+44-1223-336033; or deposit@ccdc.cam.uk).

\section{References.}

1. Abatea, C; Kolanosa, R.; Dukat, M.; Setola, V.; Roth, B. L.; Glennon, R. A. Bioorg. Med. Chem. Lett. 2005, 15,3510

2. a) Li, J.; Wang, T.; Yu, P.; Peterson, A.; Weber, R.; Grubisha, D.; Bennett, D.; Cook, J. M. J. Am. Chem. Soc. 1999, 121, 6998 b) Yu, P.; Cook, J. M. J. Org. Chem. 1998, 63, 9160

3. Franchi, P.; Lucarini, M.; Mezzina, E.; Pedulli, G. F. J. Am. Chem. Soc. 2004, 126, 4343

4. SAINT, Manual Version 5/6.0, Bruker Analytical X-ray Systems Inc.: Madison, Wisconsin, 1997

5. SHELXTL-NT, Manual Version 5.1, Bruker Analytical X-ray Systems Inc.: Madison, Wisconsin, 1997 


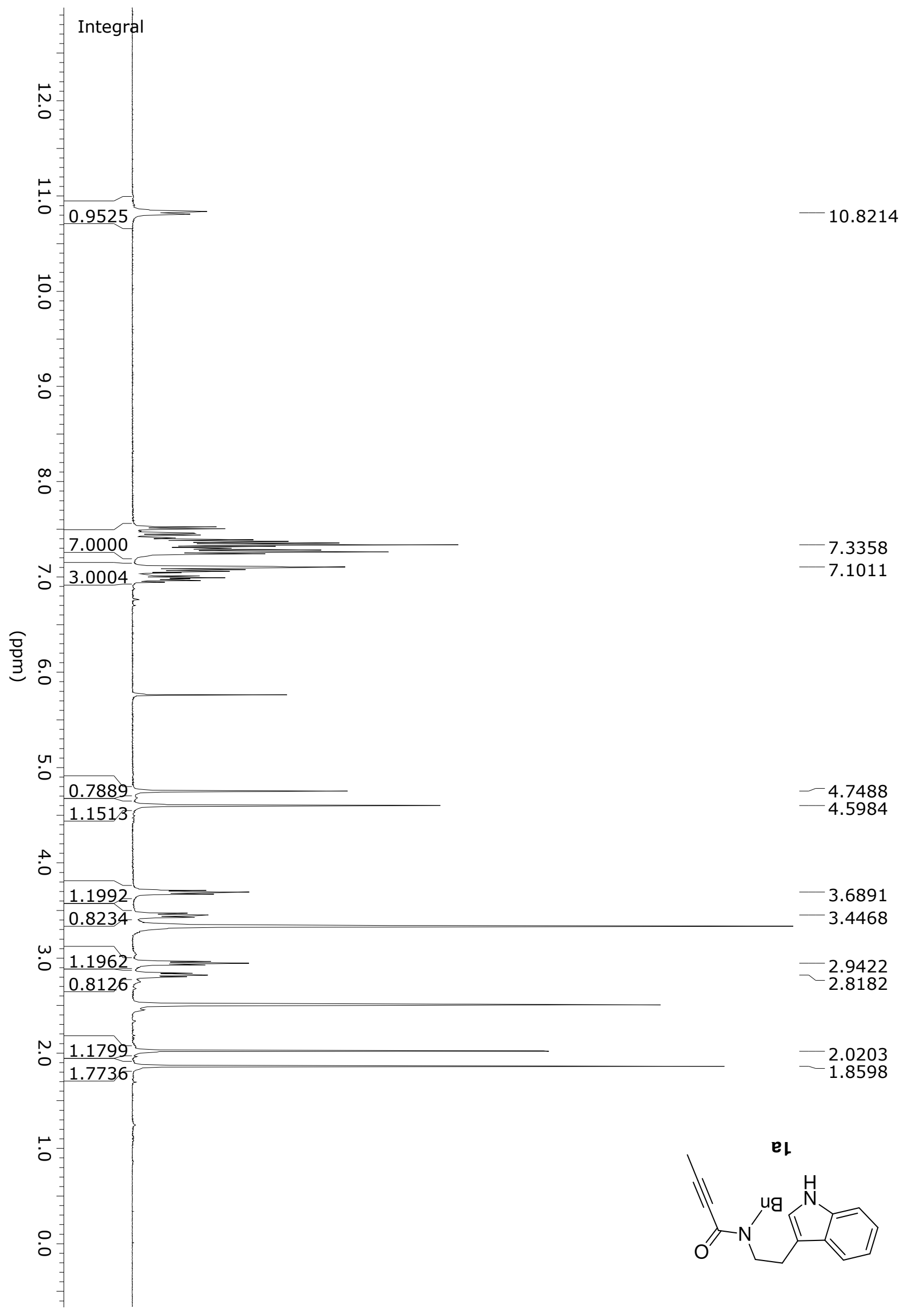




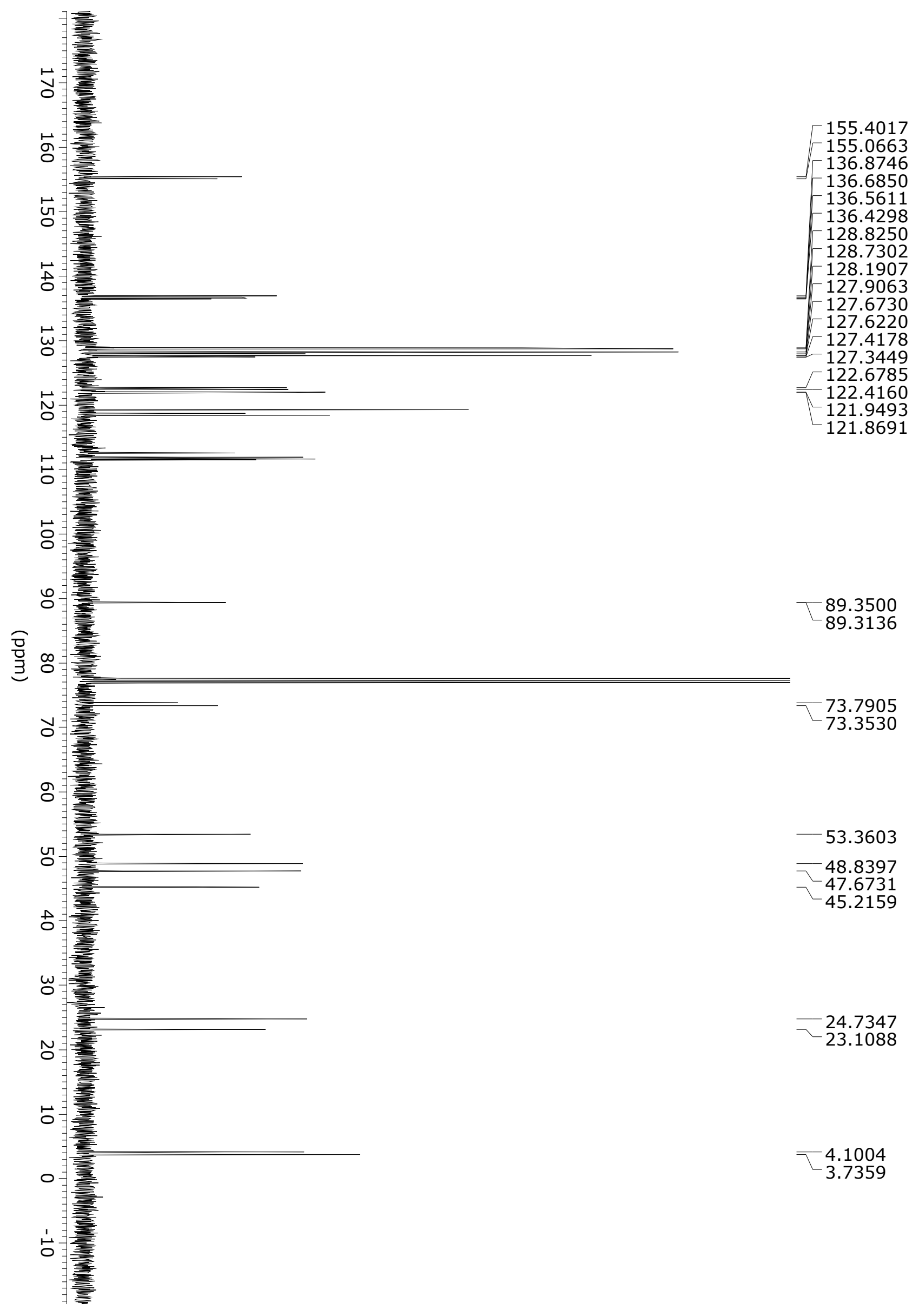




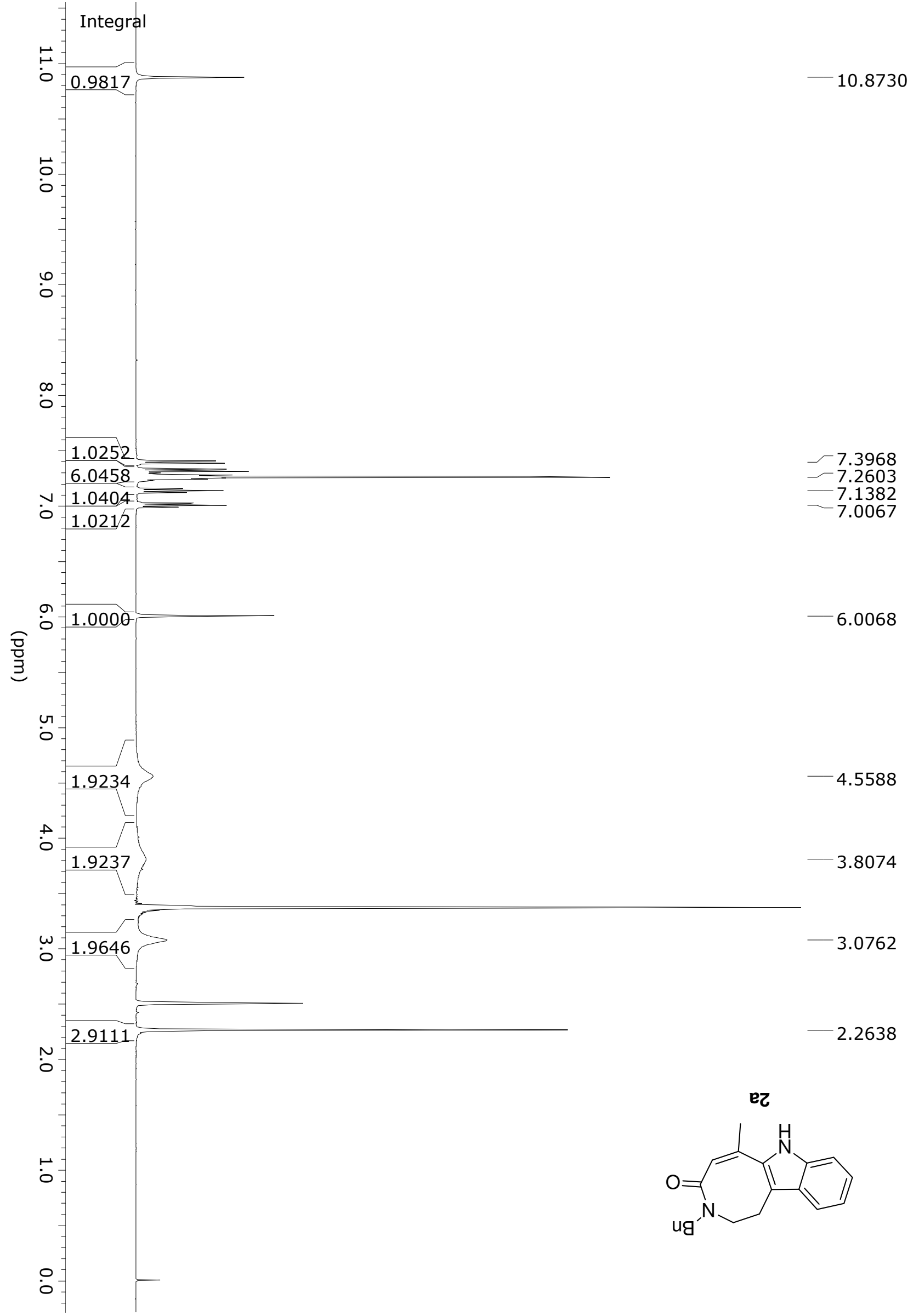




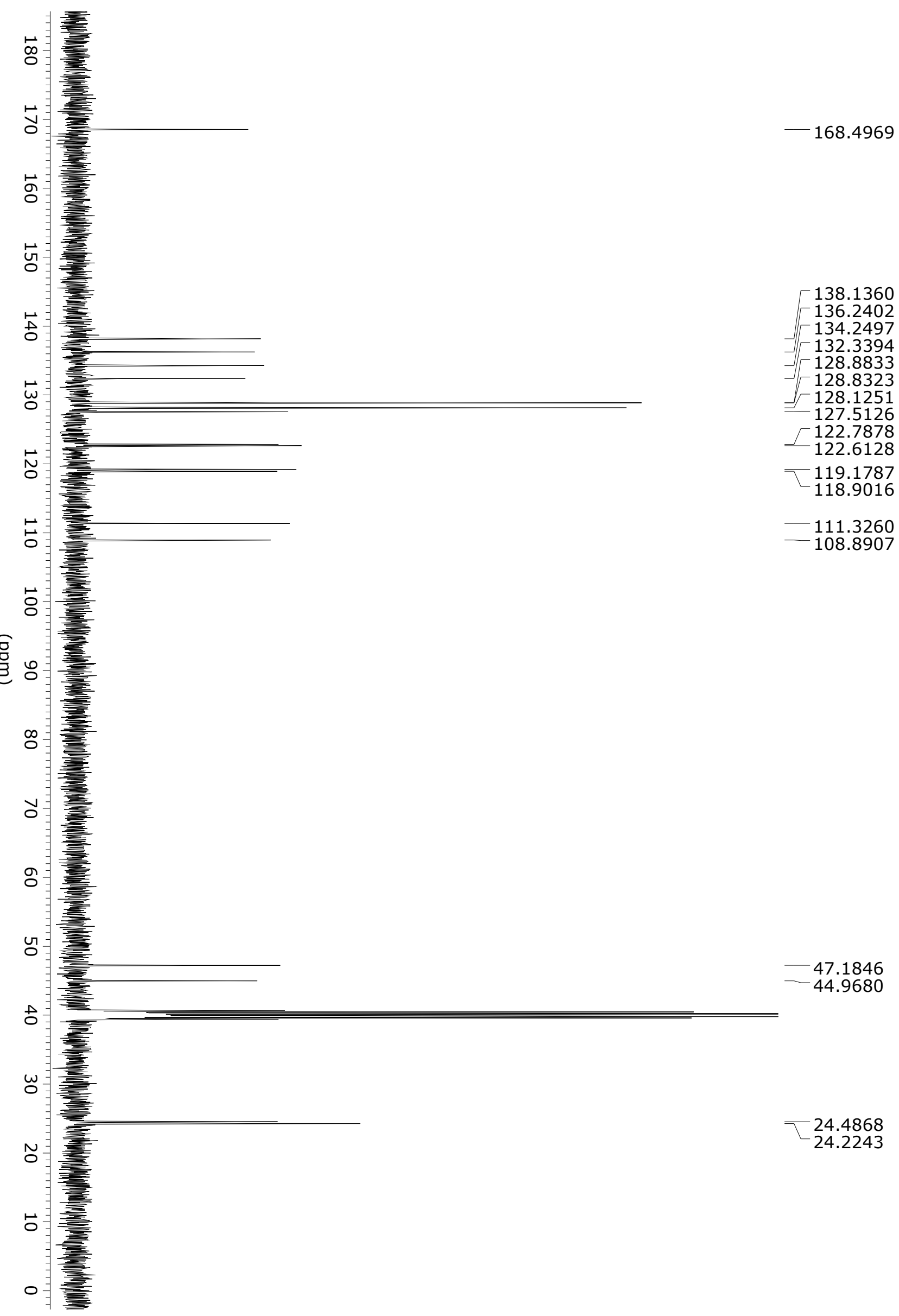




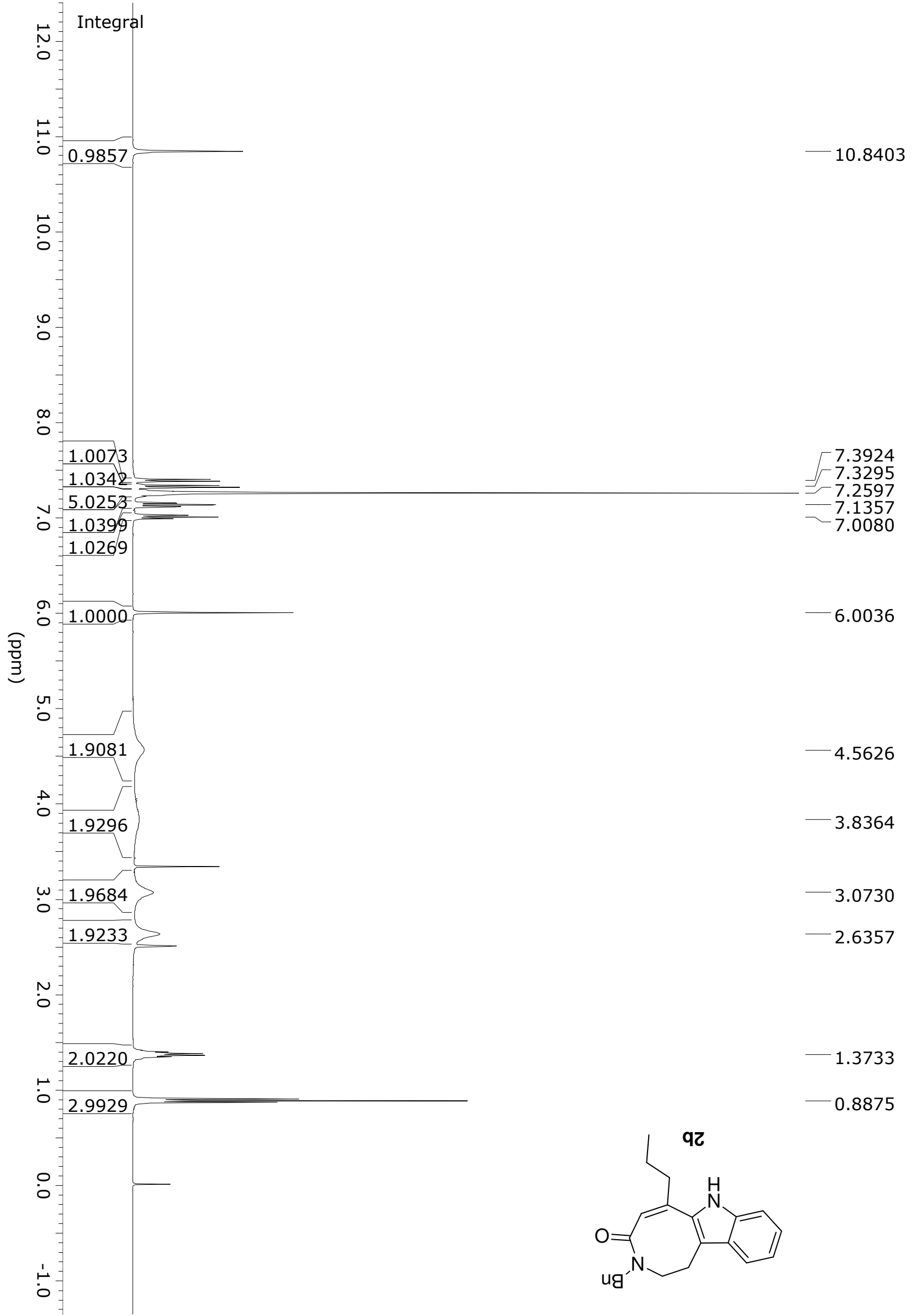




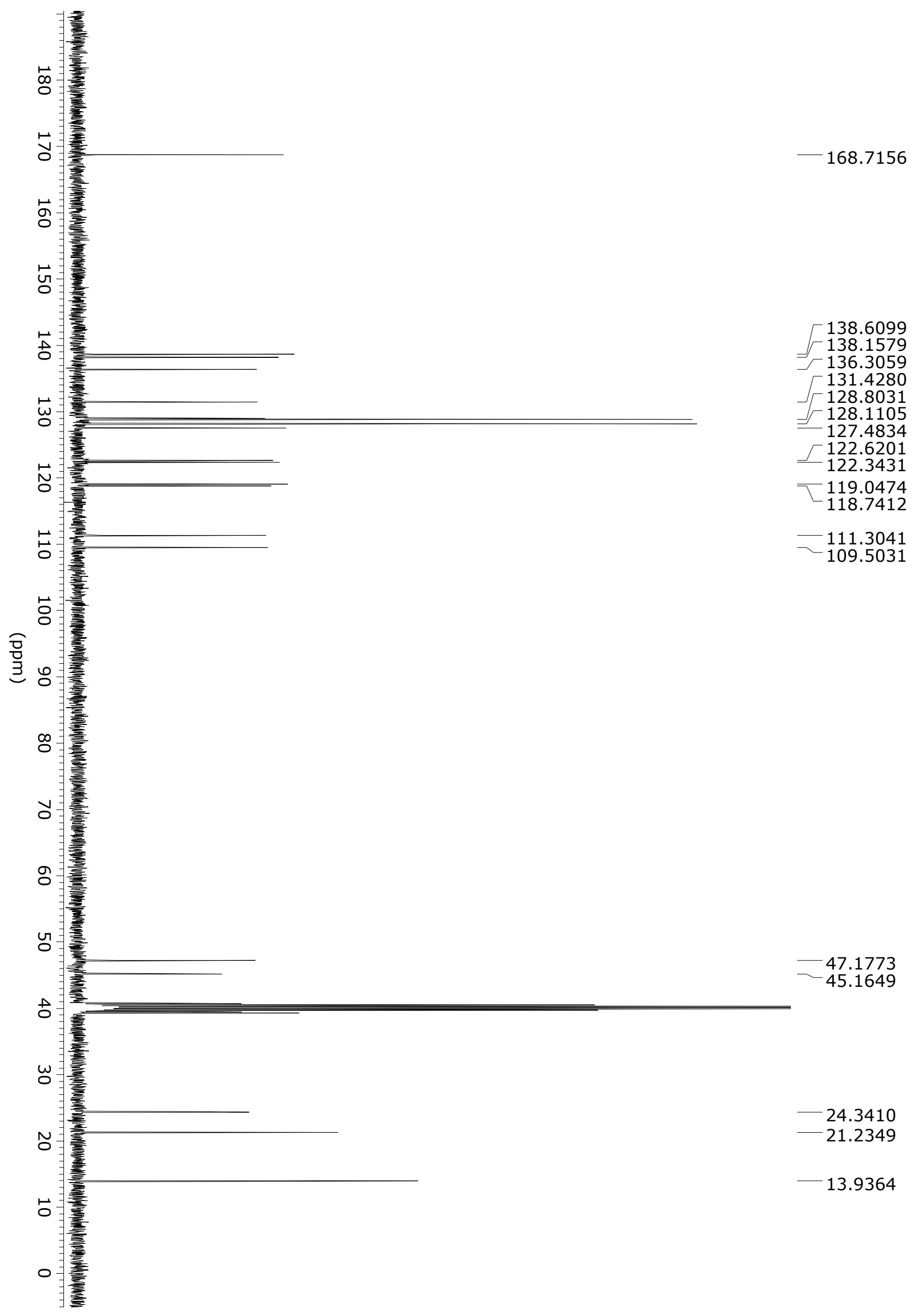




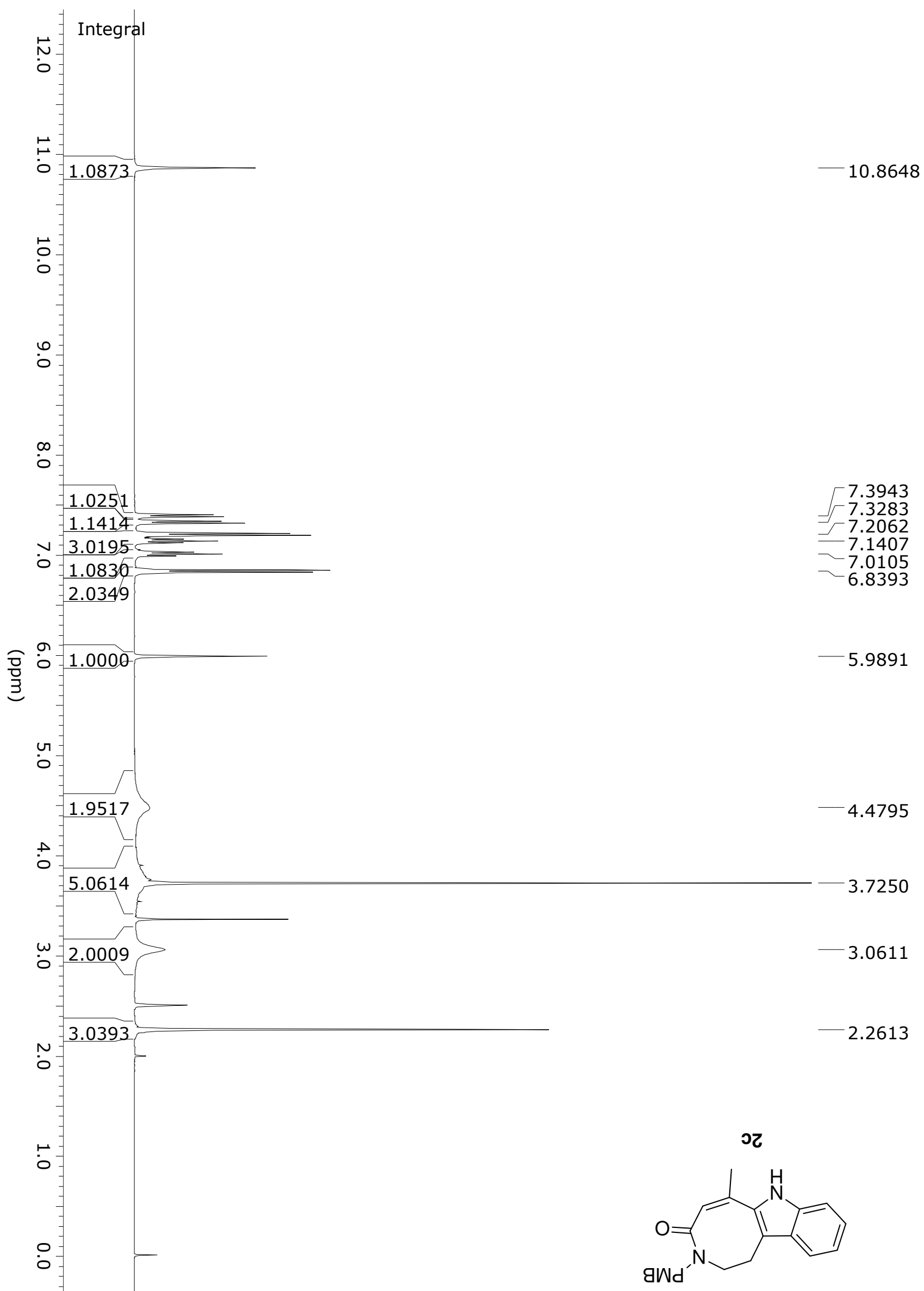




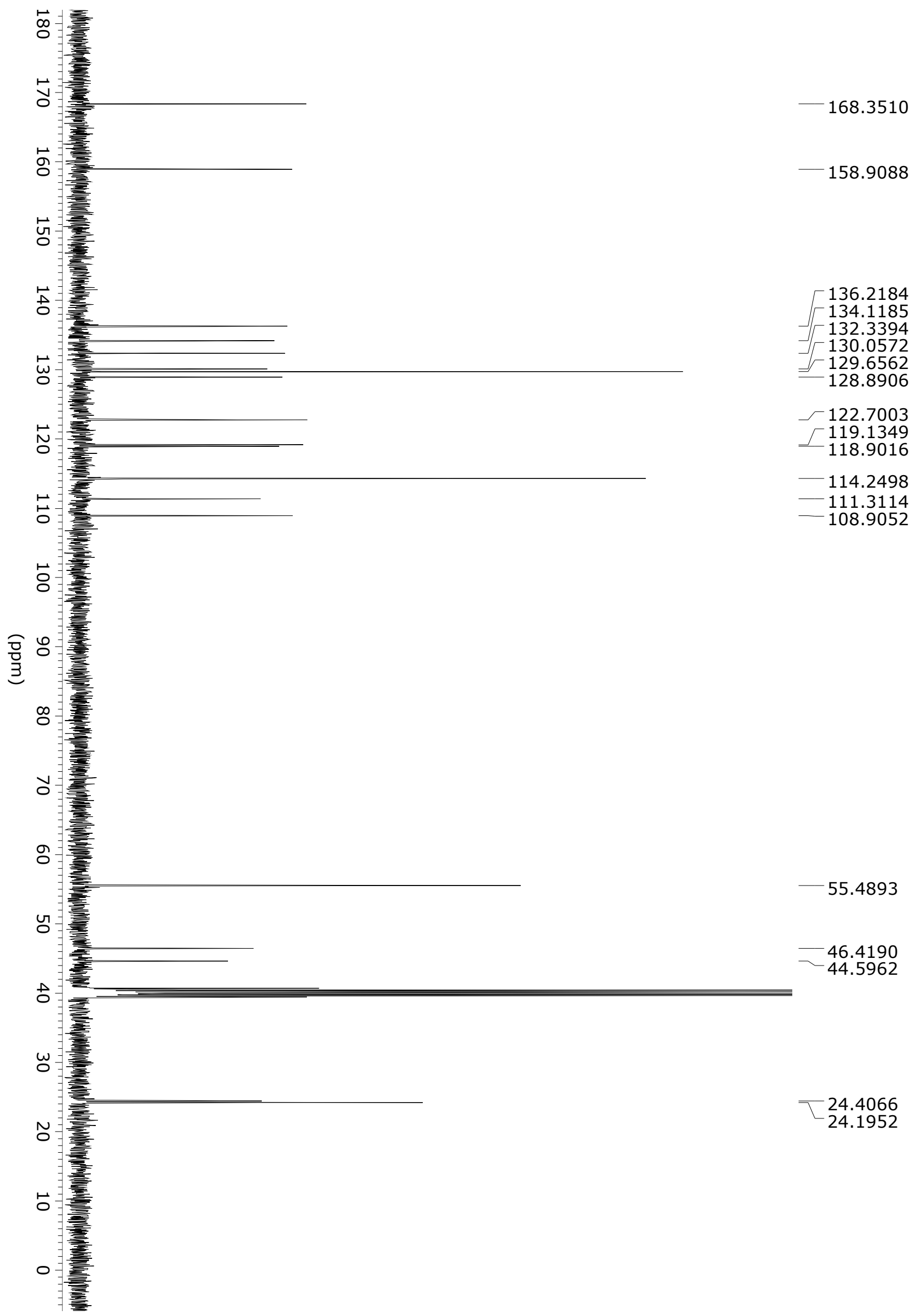




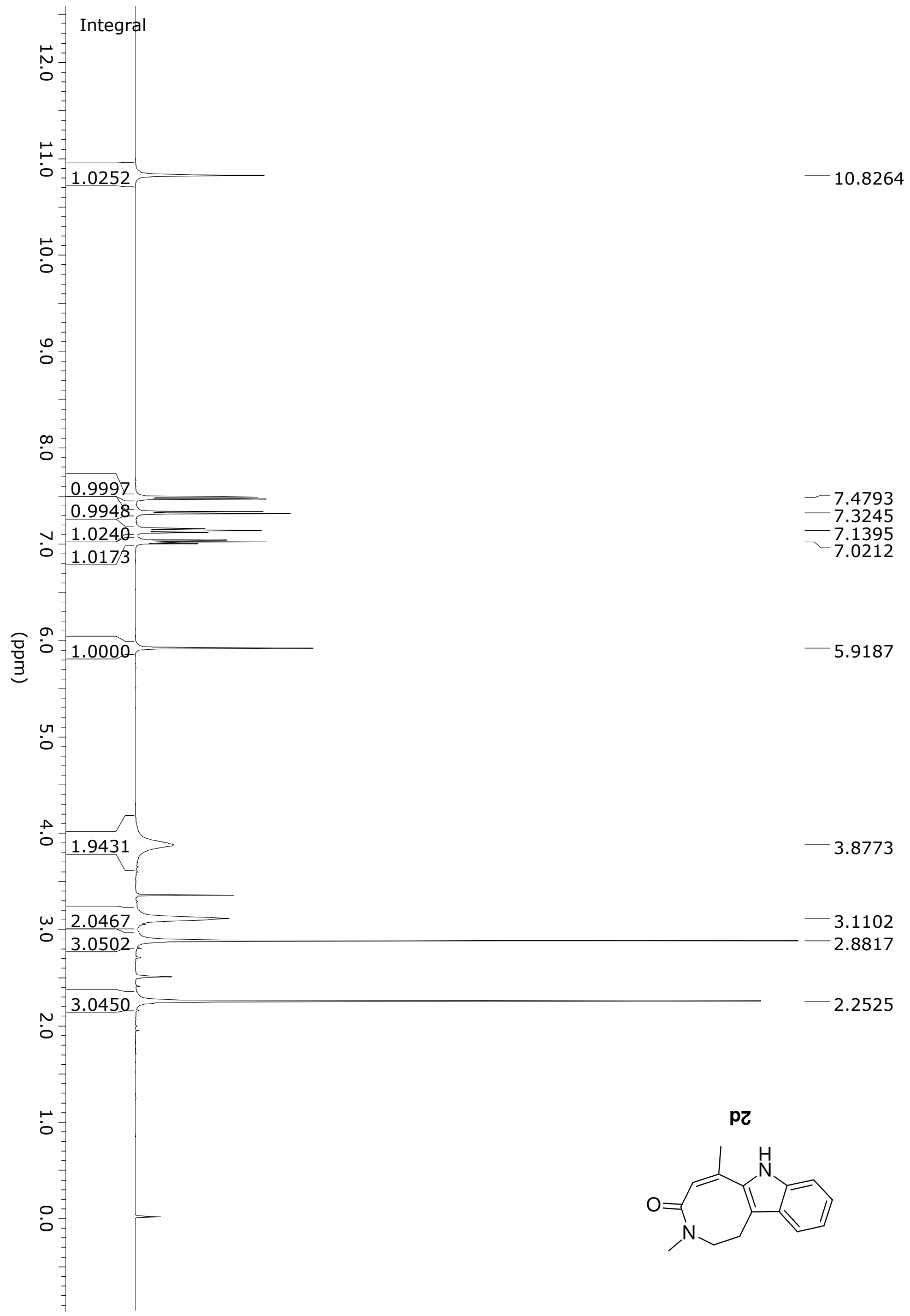


品

ํ

$-168.1469$

응

जั

古

$-136.2111$

$-133.9435$

茨

$-132.3175$

$-128.9490$

$-122.8972$

$-122.7587$

$-119.1932$

등

$\begin{array}{r}119.1932 \\ \hline\end{array}$

官

$-111.3041$

$-108.7011$

응

휠

$\infty$

○

g

U

$-47.4252$

b

章

w

32.0771

ㅇ.

24.3629

24.1296

늠

○ 


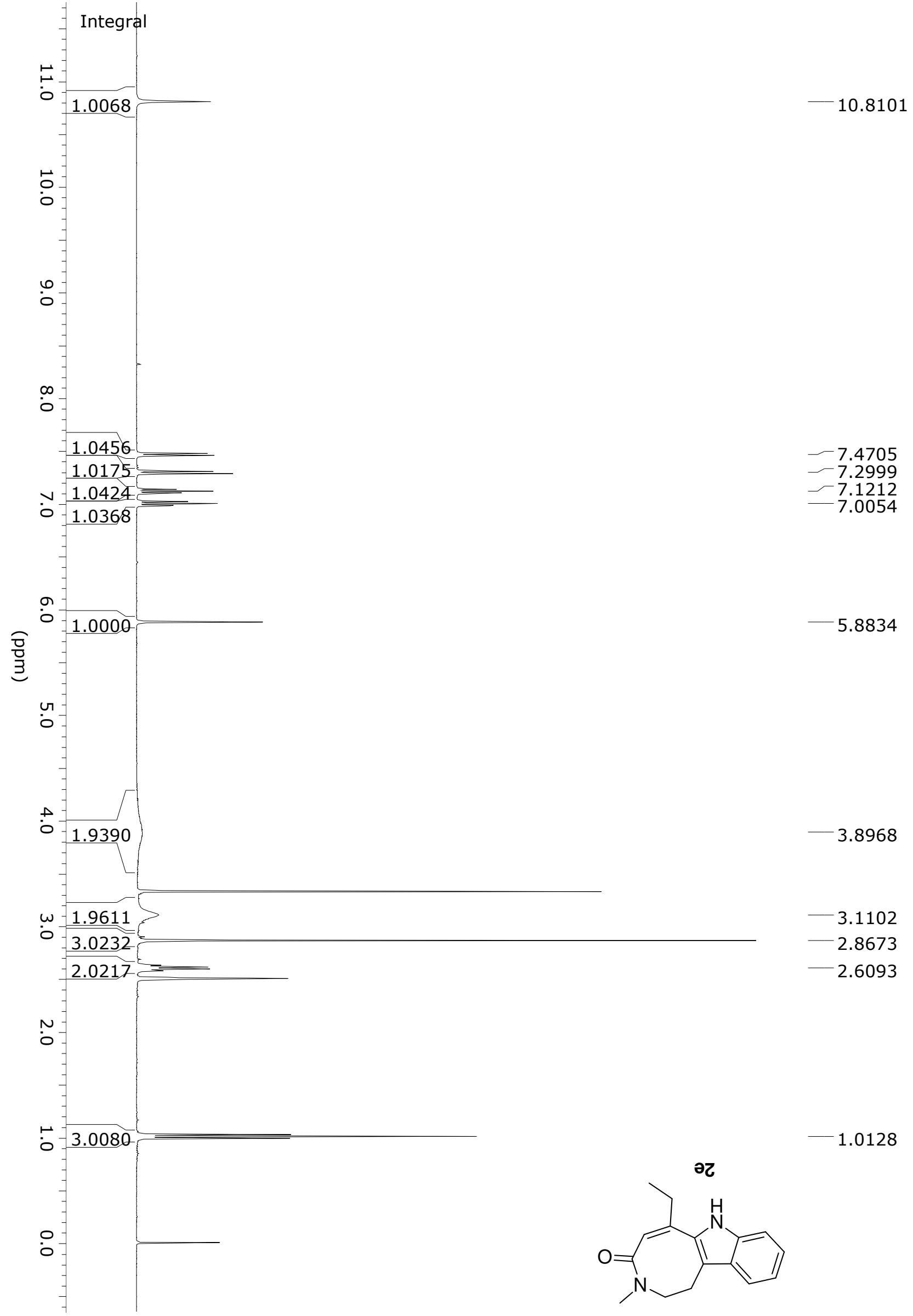




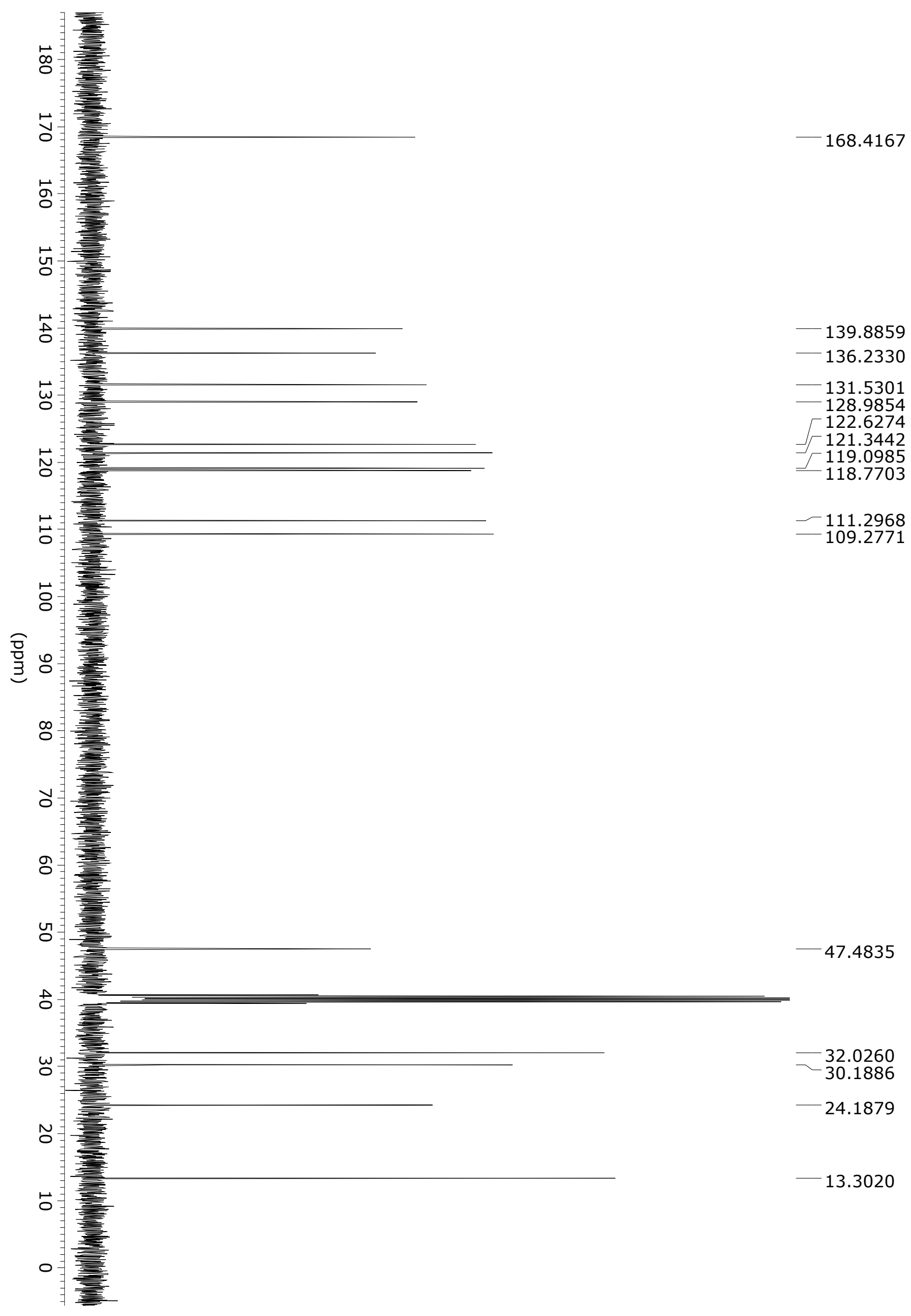




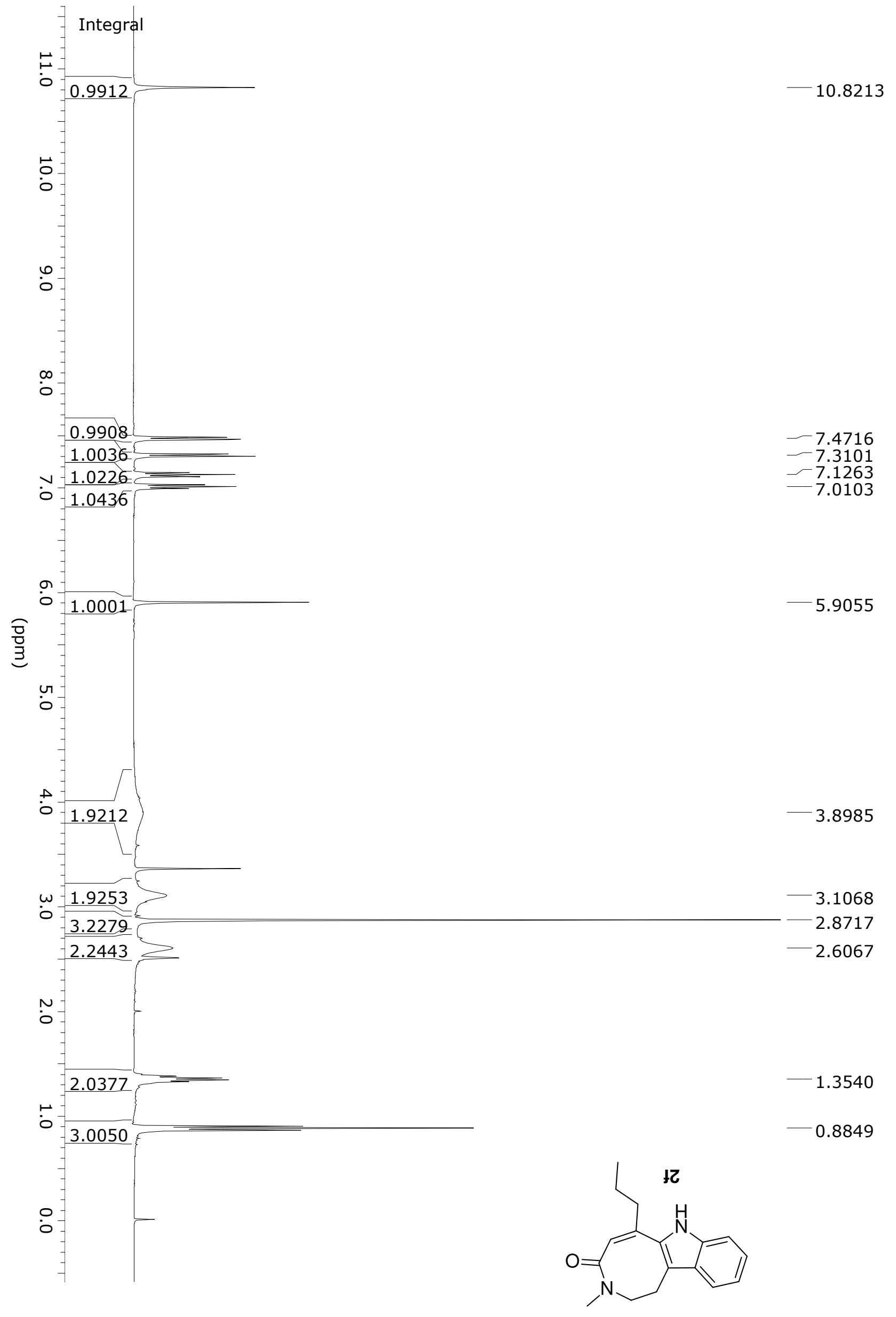




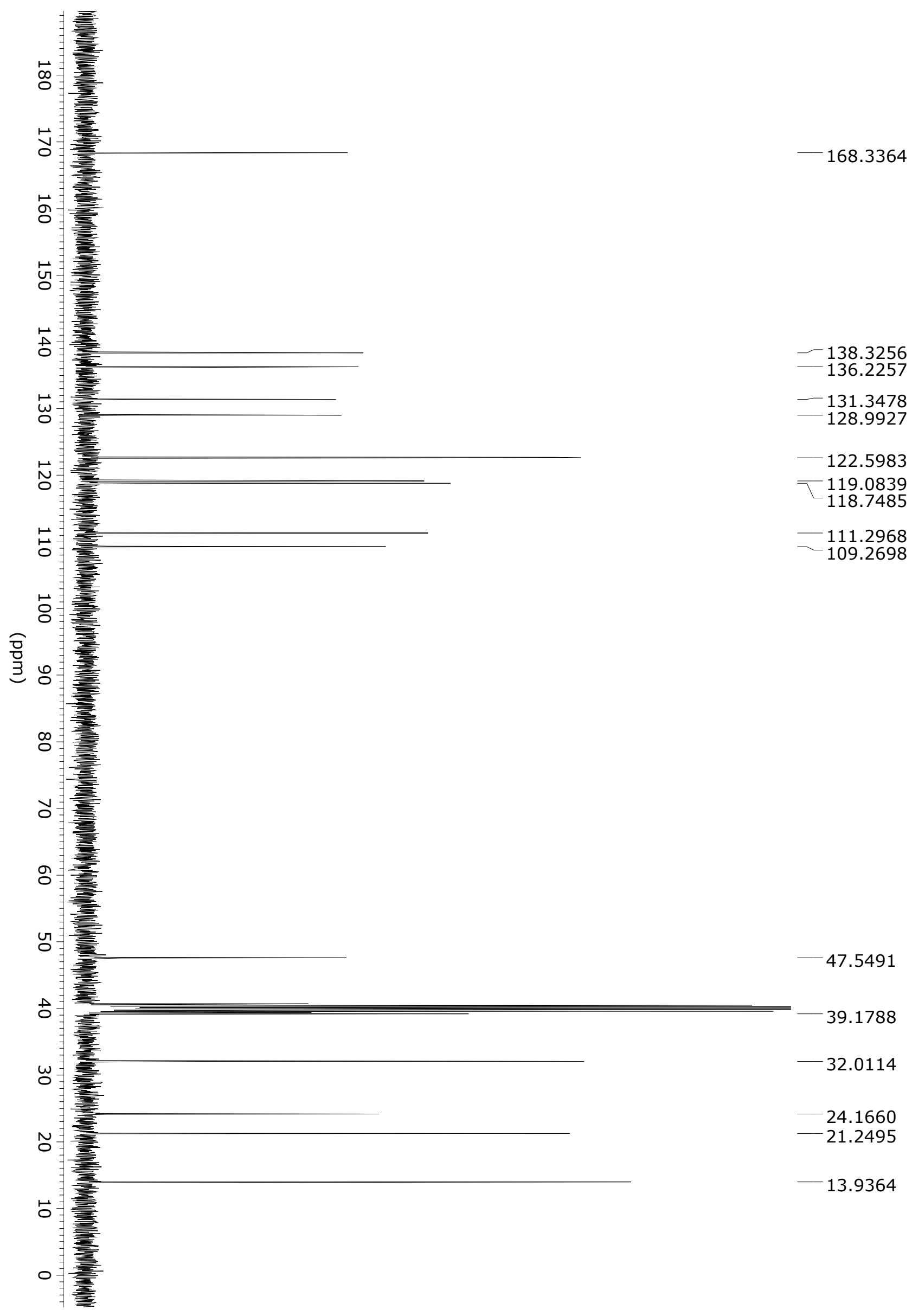




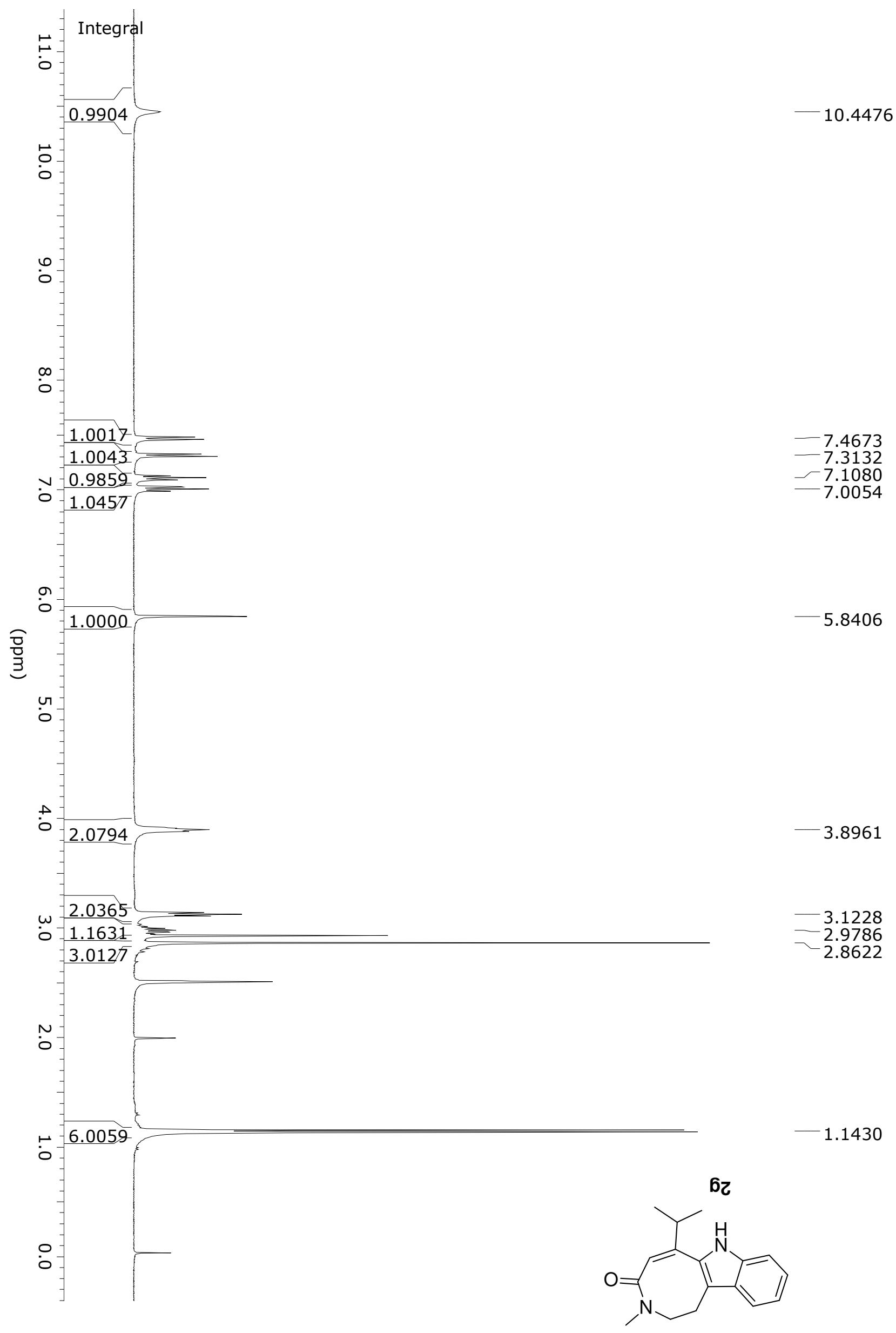


宓

$\stackrel{5}{0}$

잉

जั

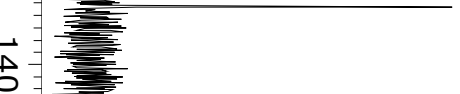

$-144.6835$

$-136.6340$

$\stackrel{\omega}{\omega}$

$-132.5071$

- 129.1604

122.2920

120.3380

$-119.0547$

茫

118.3839

官

$-111.3551$

$-108.9782$

安

훌

$\infty$

○

g

등

47.7825

b

w

$-33.5572$

31.9458

- 24.0129

N

21.9641

능

○ 


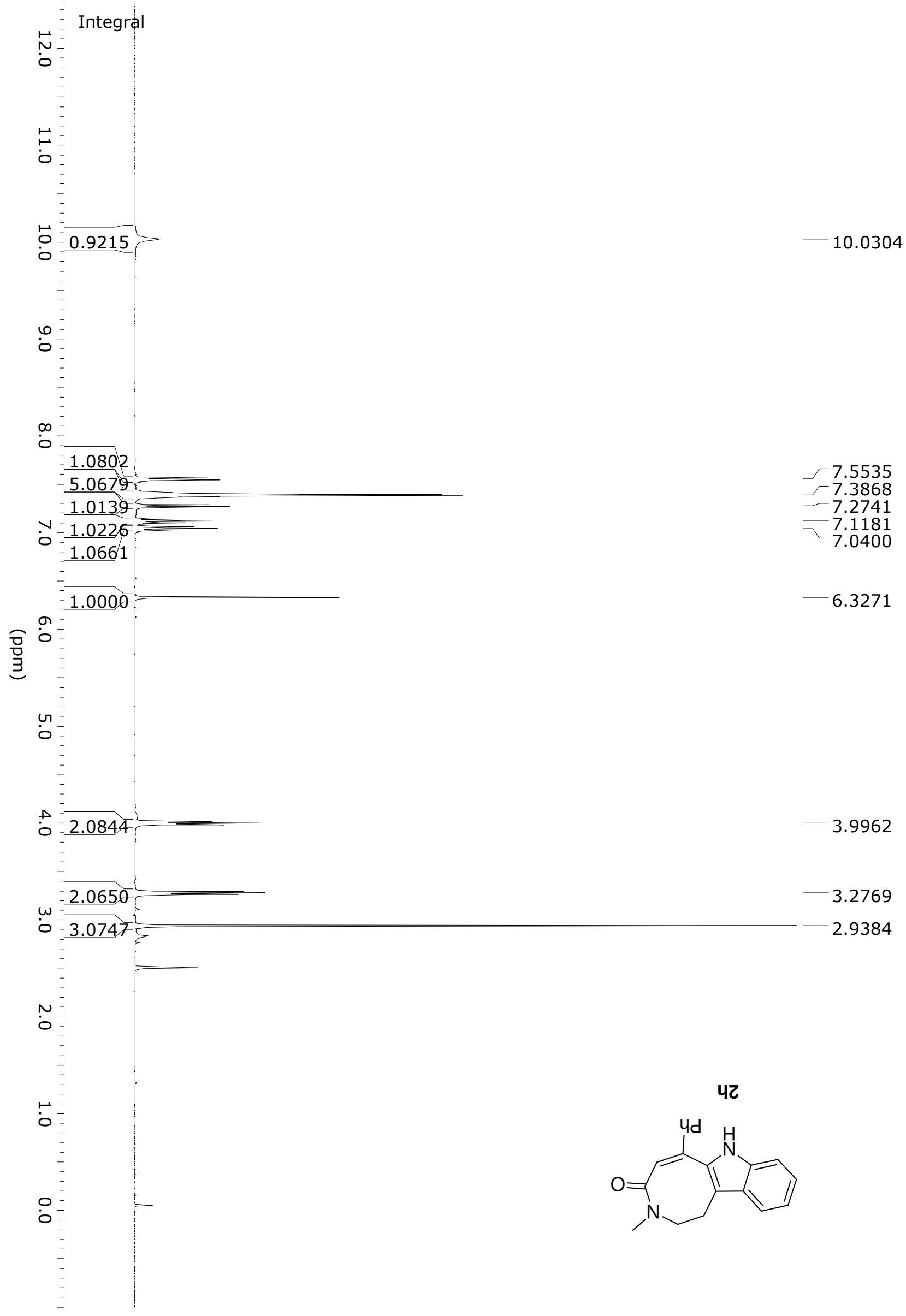




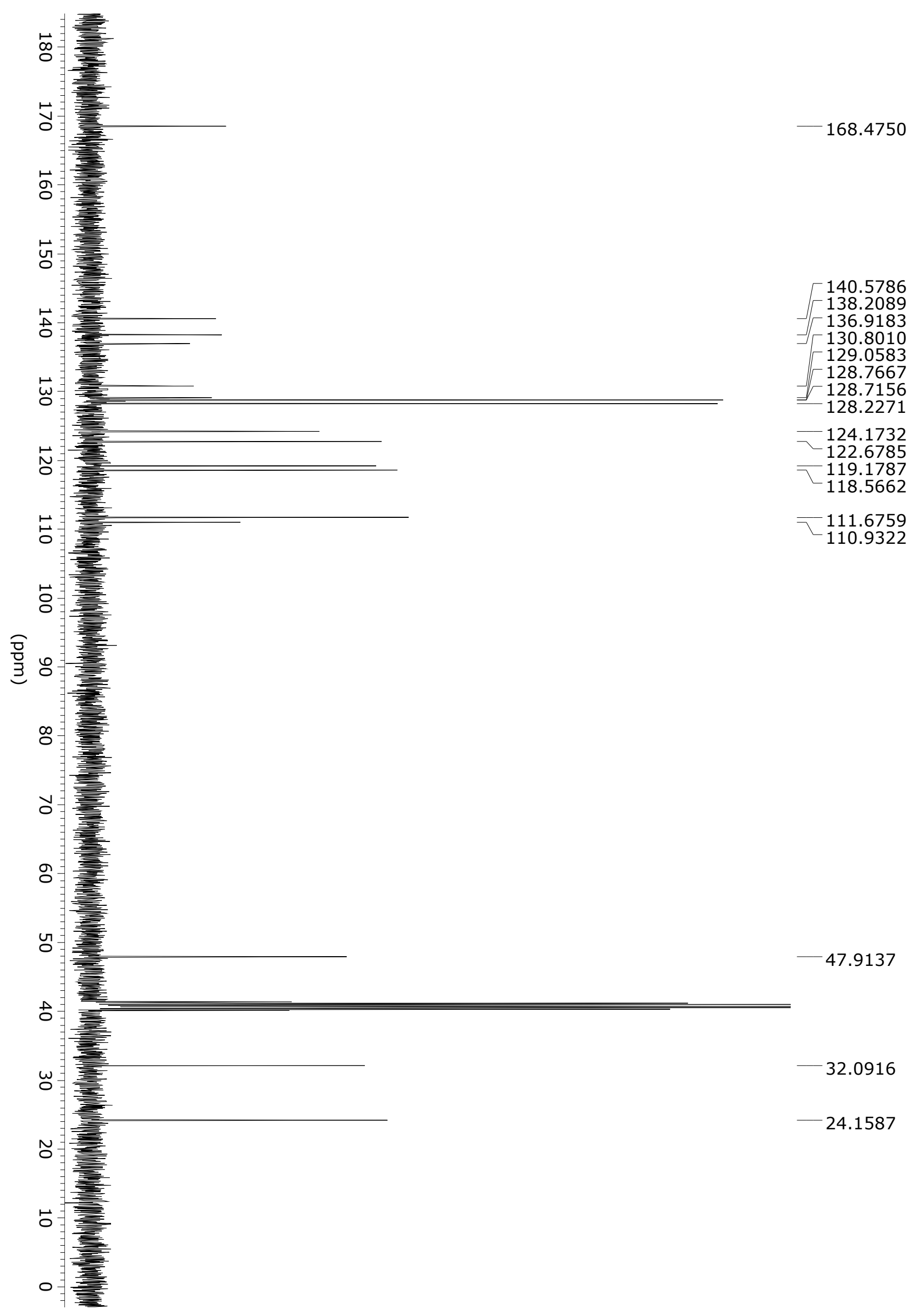




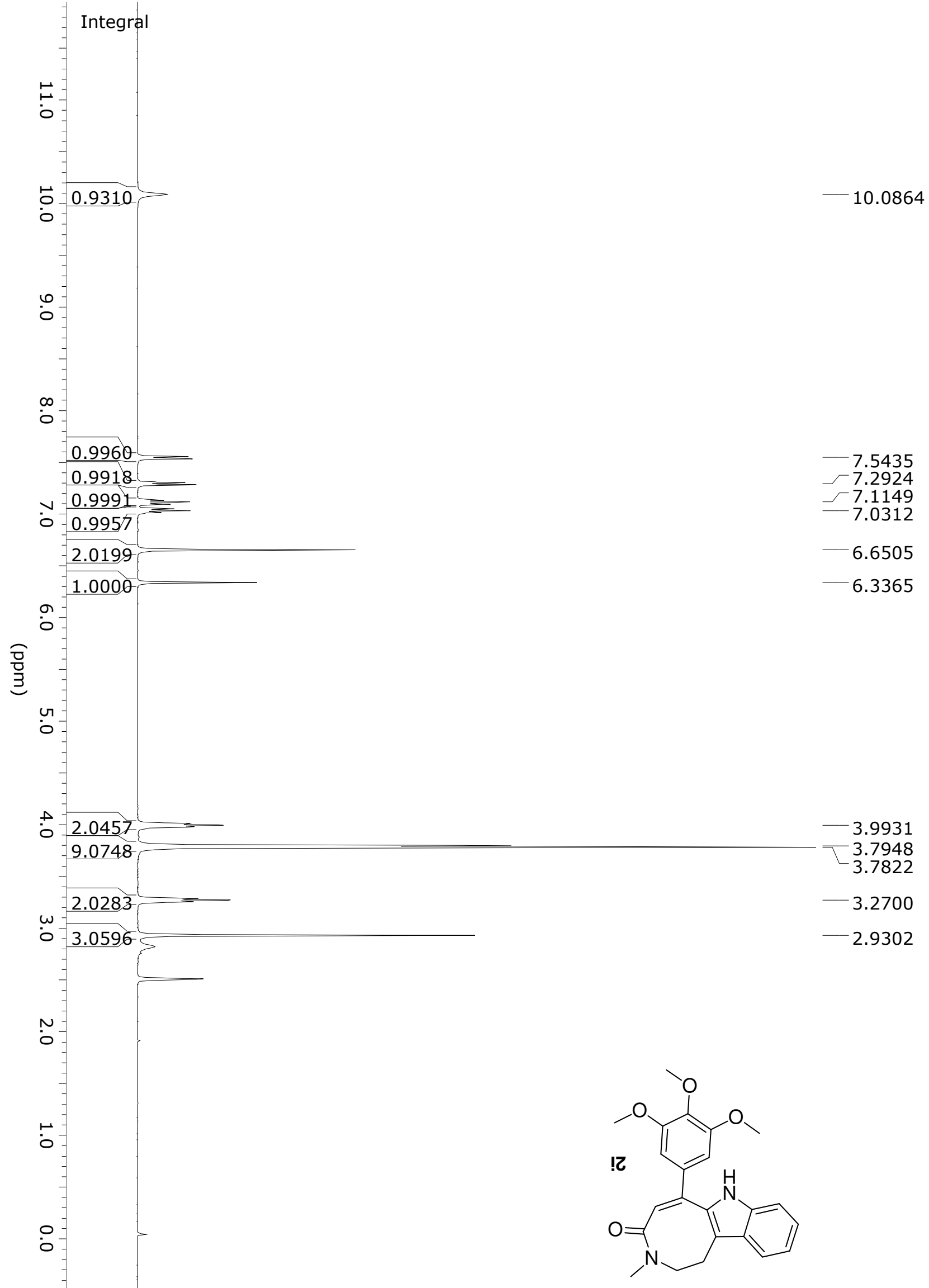




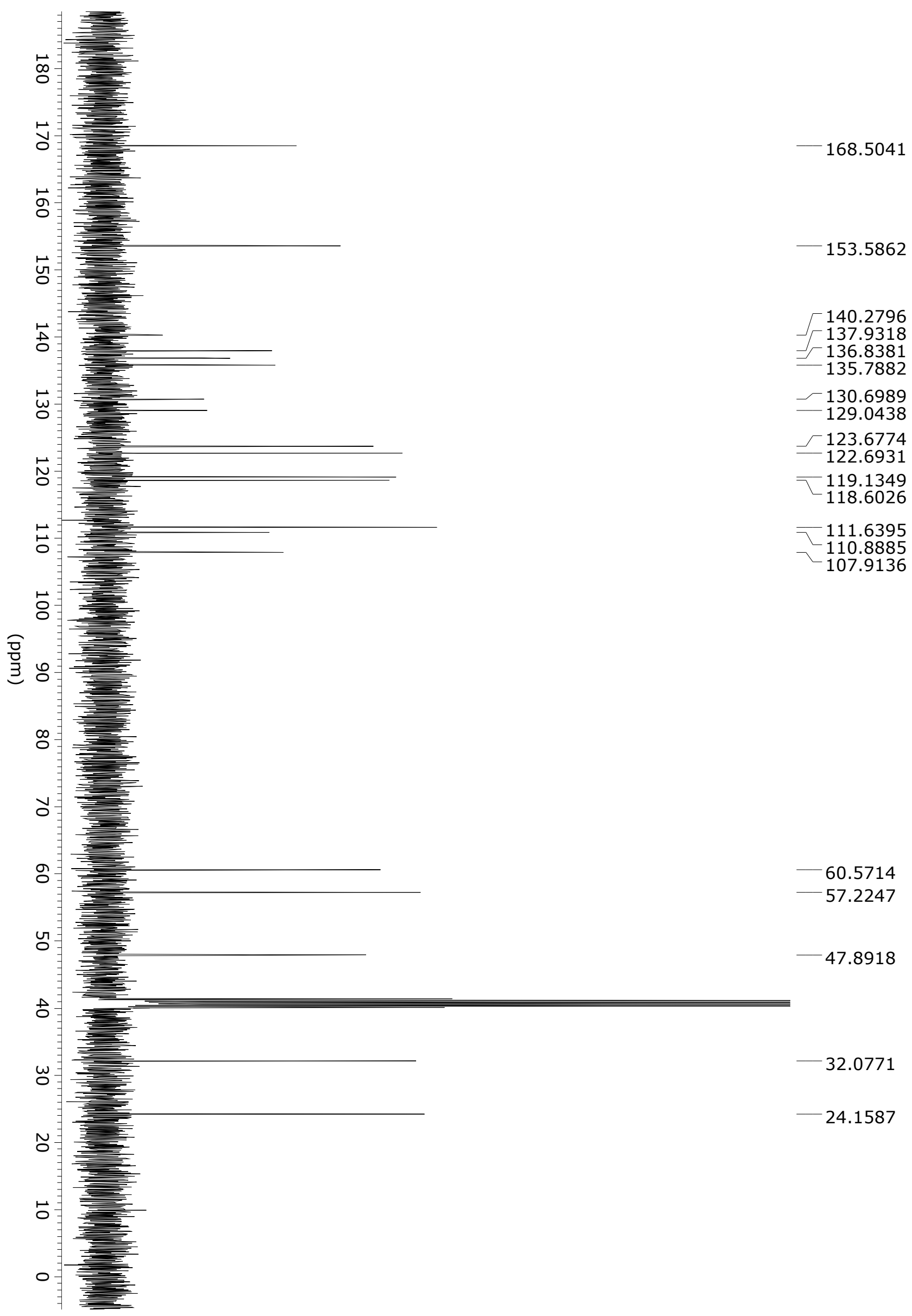




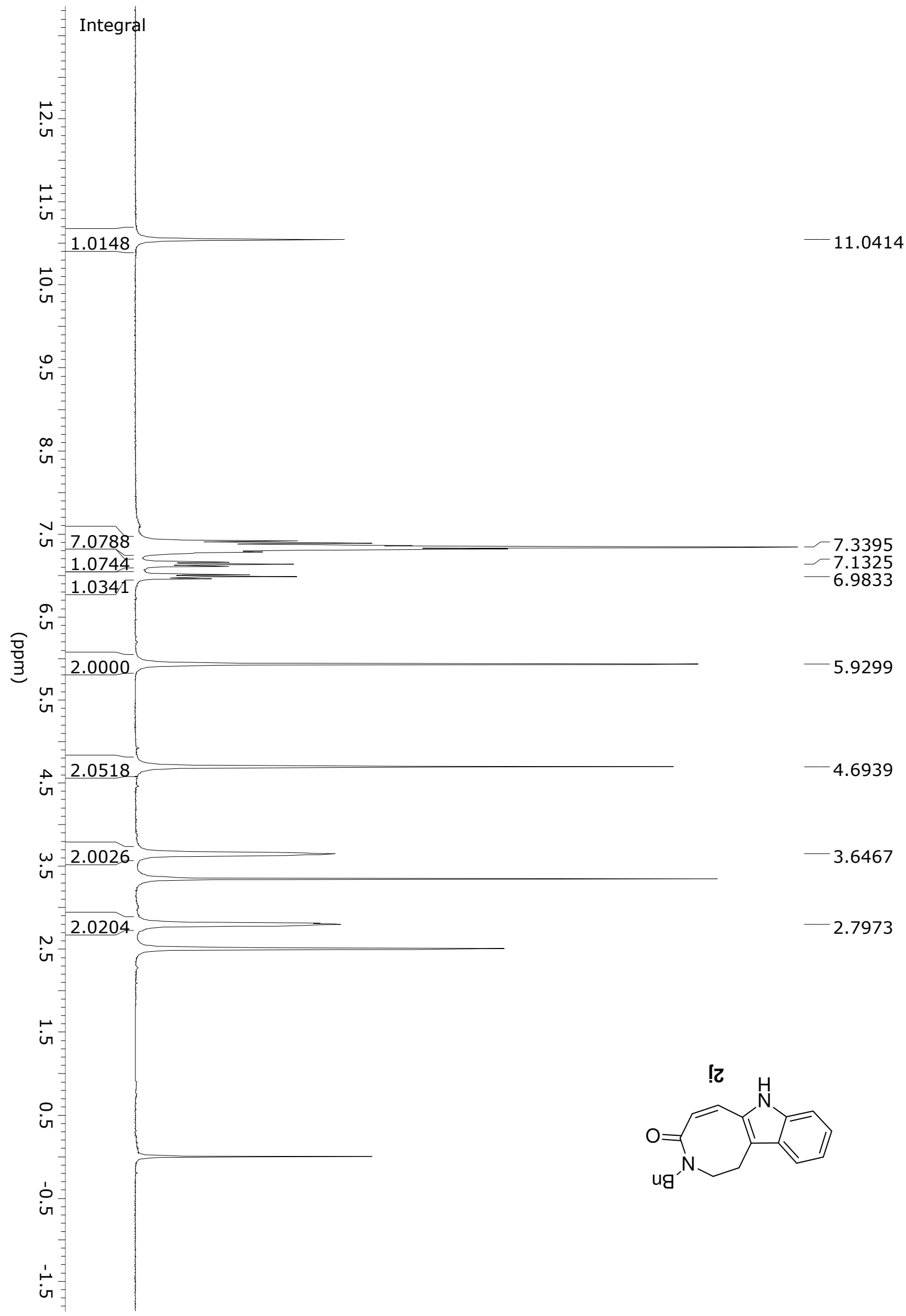




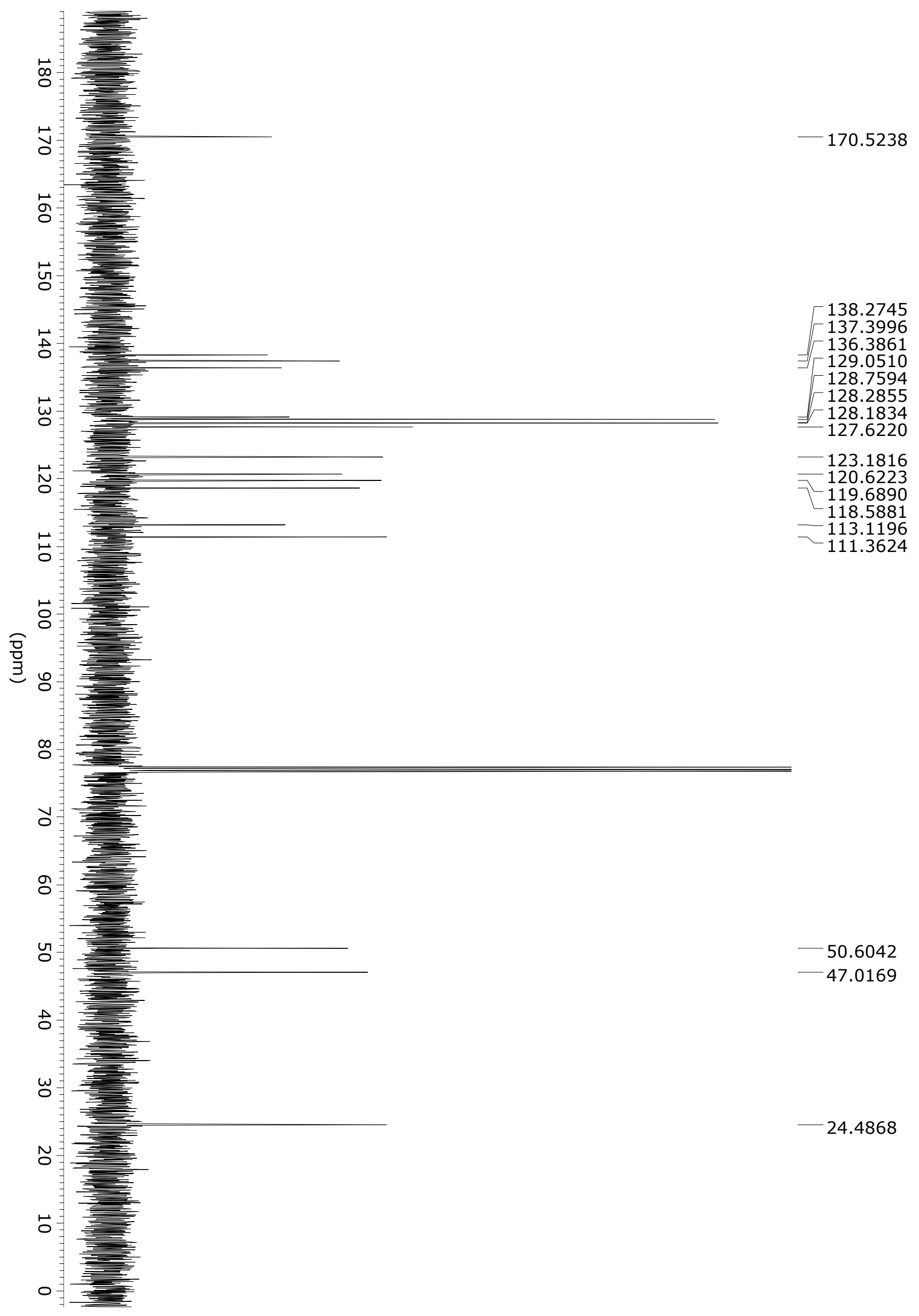




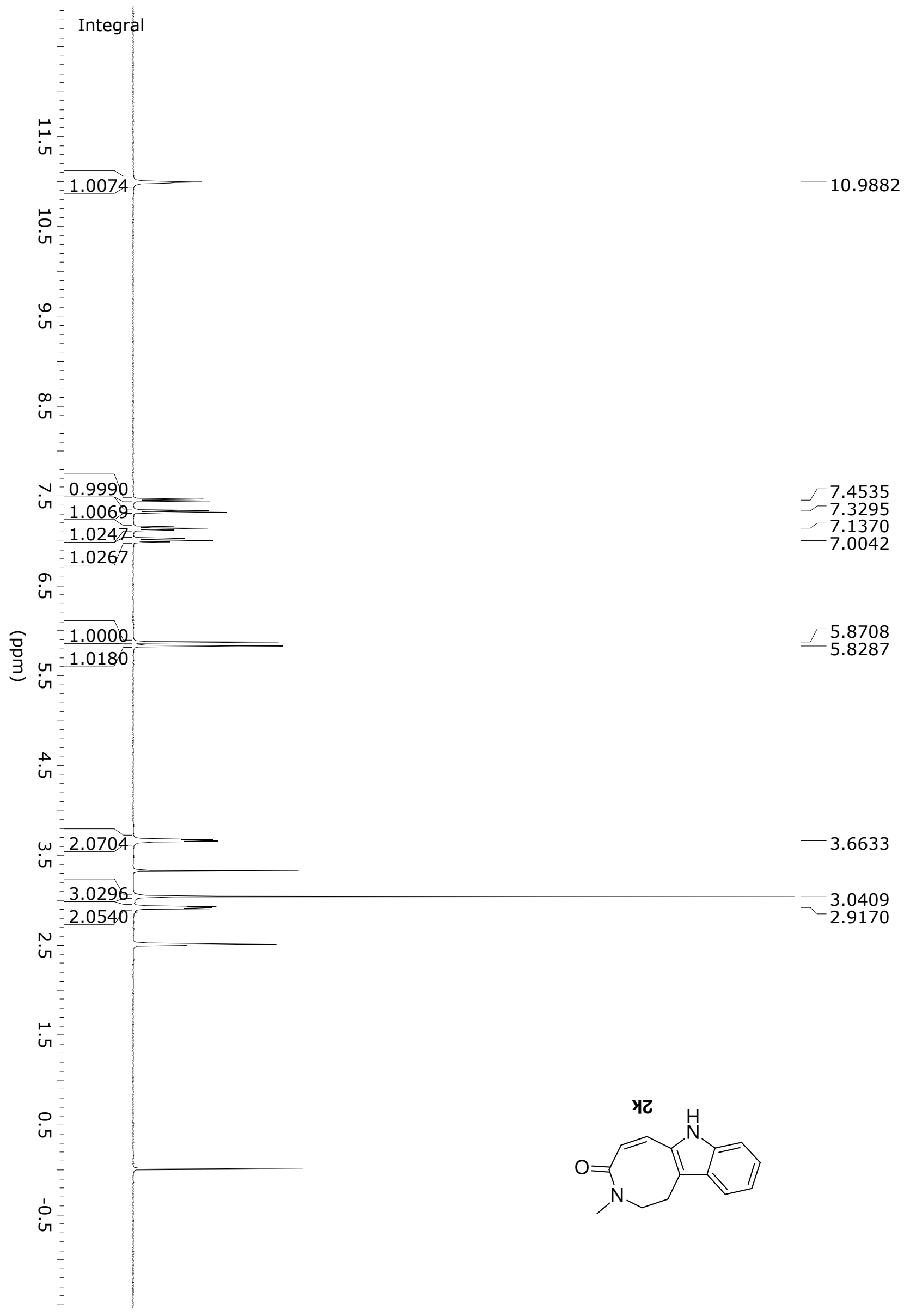




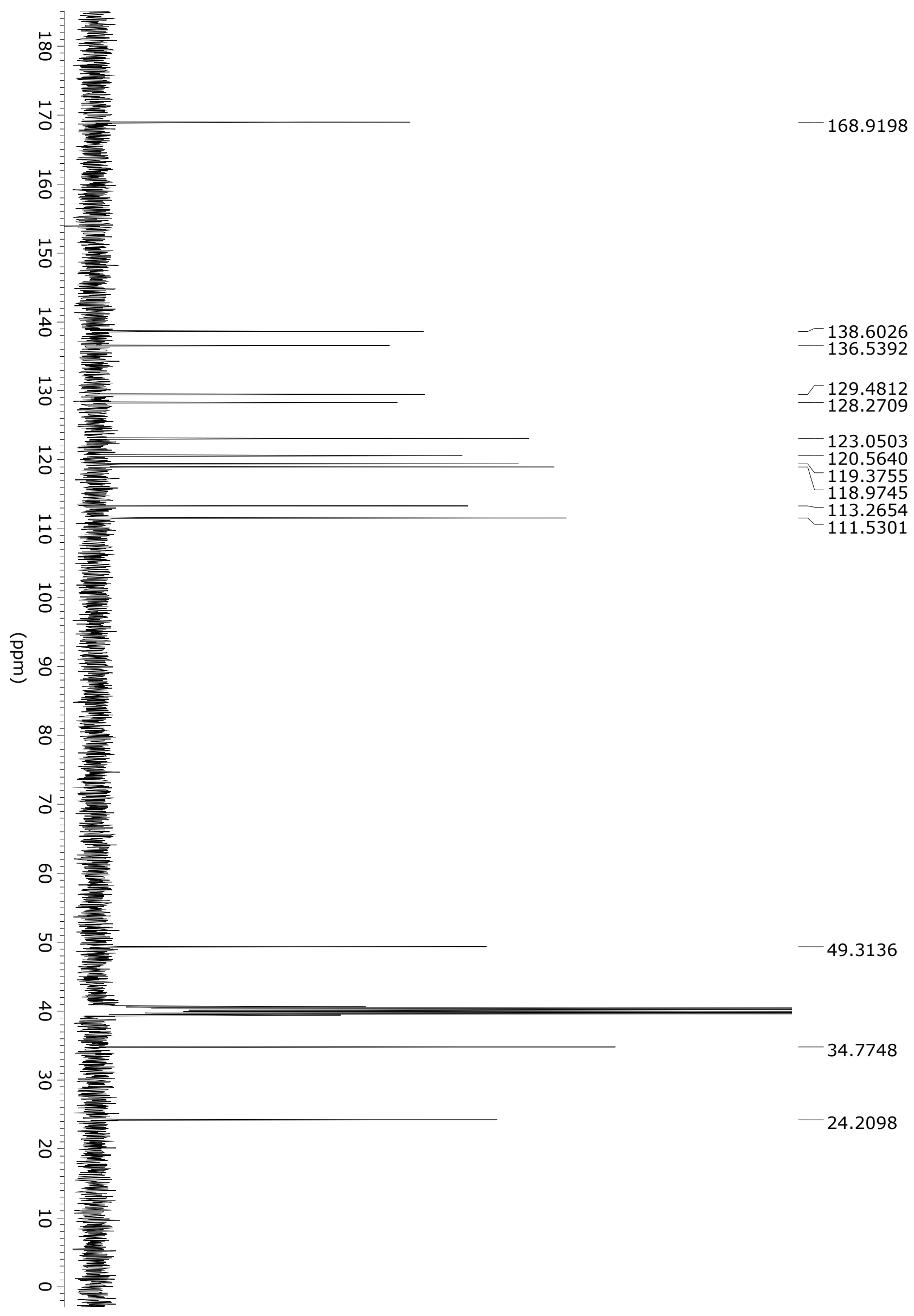




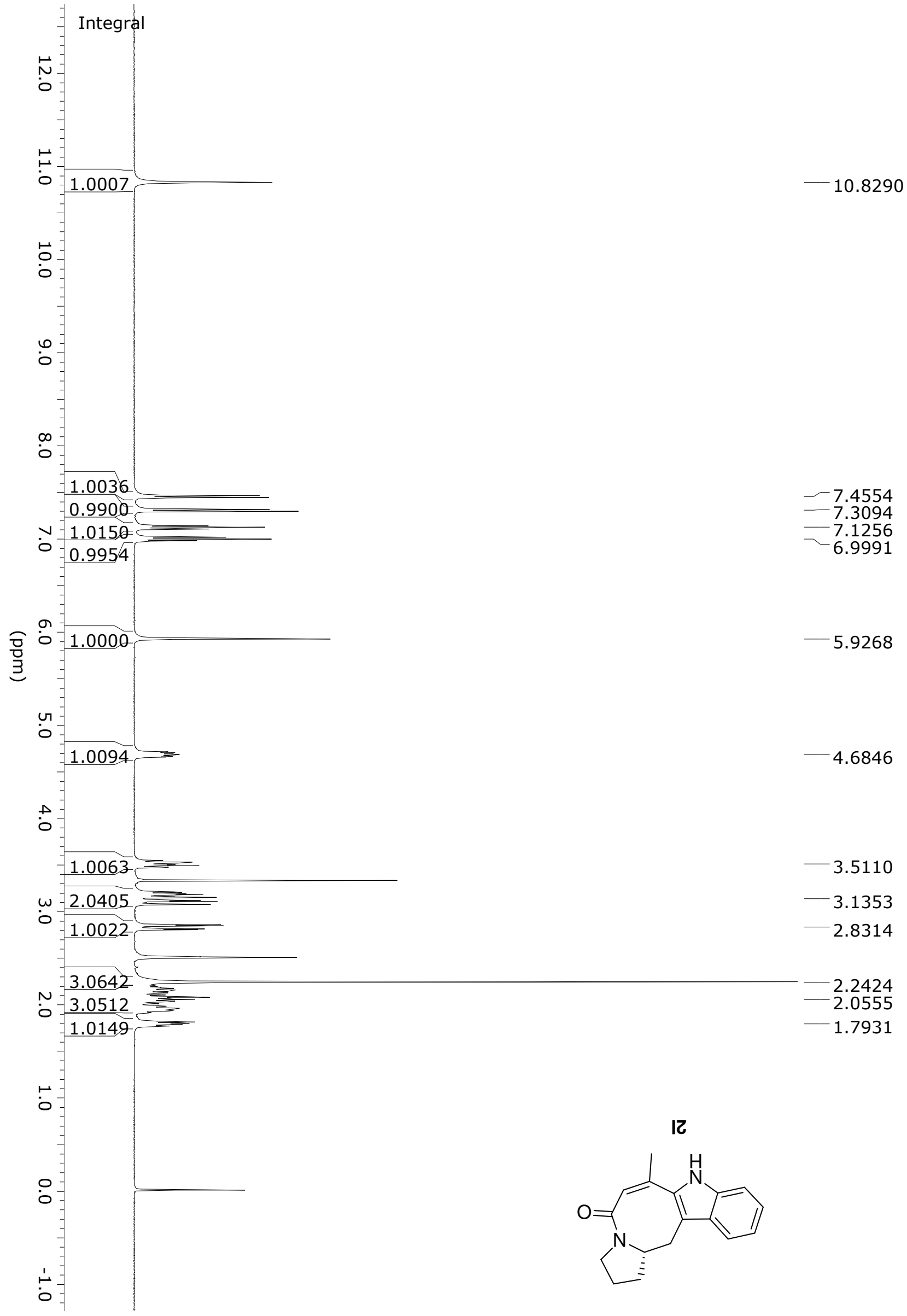




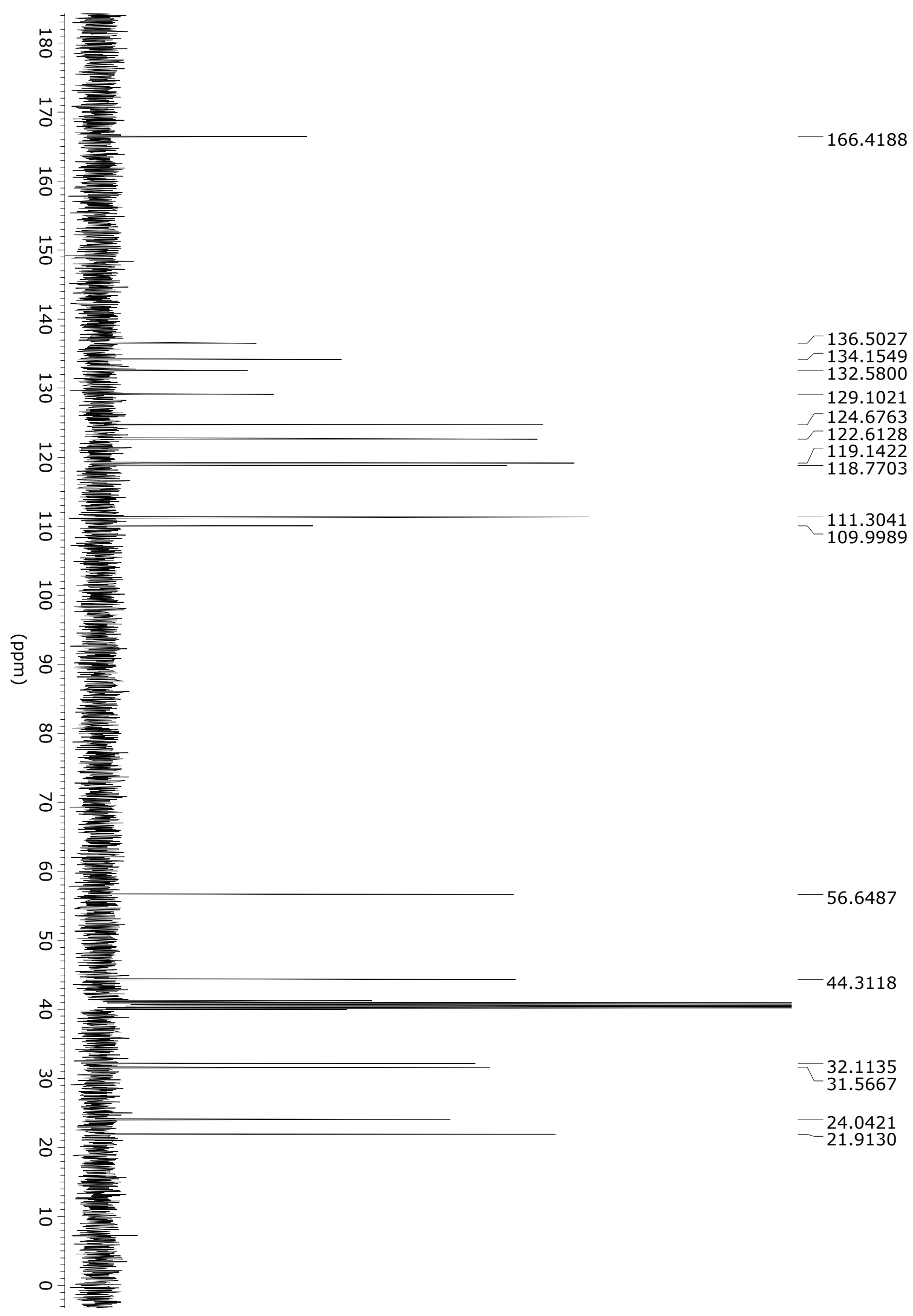




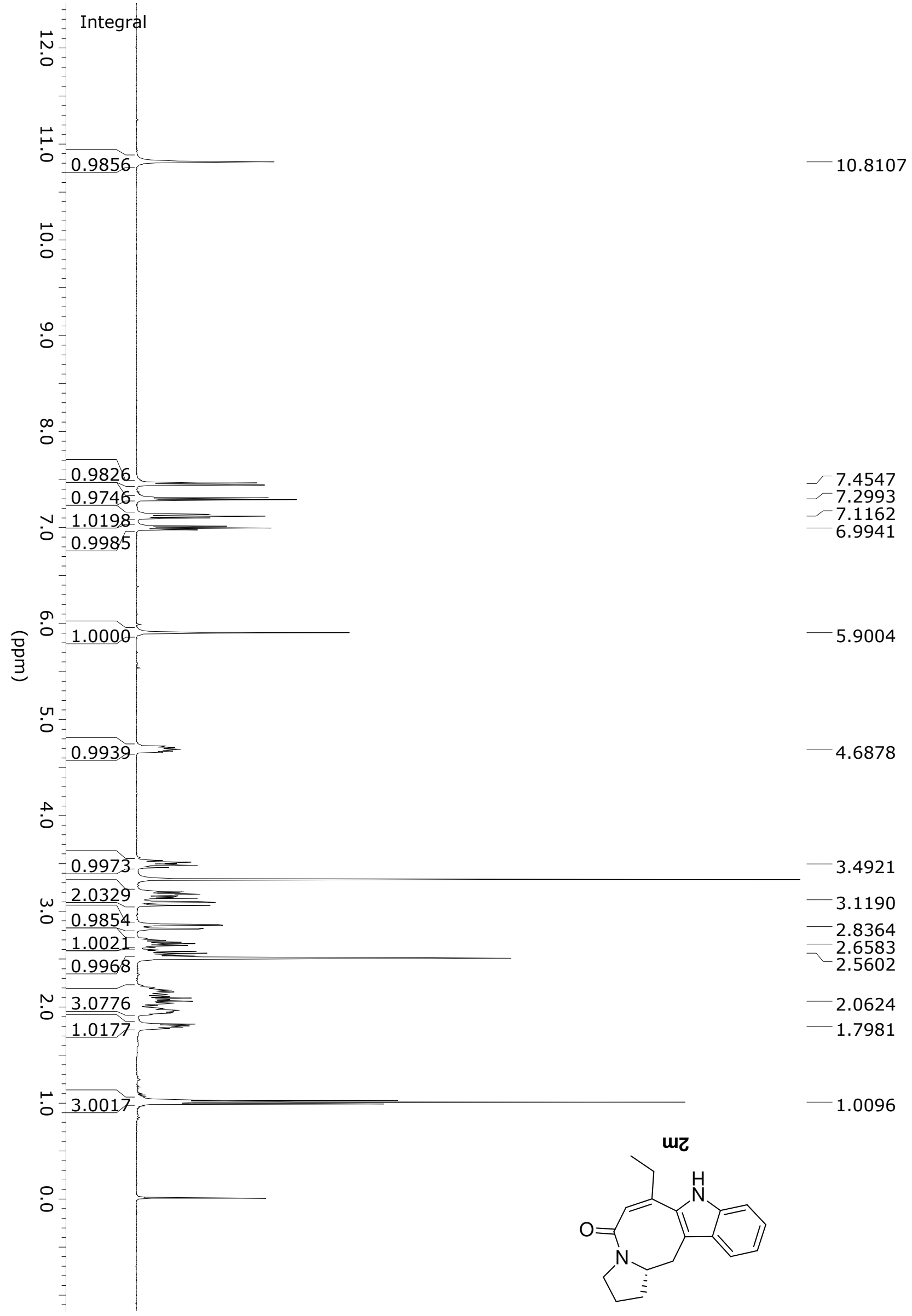




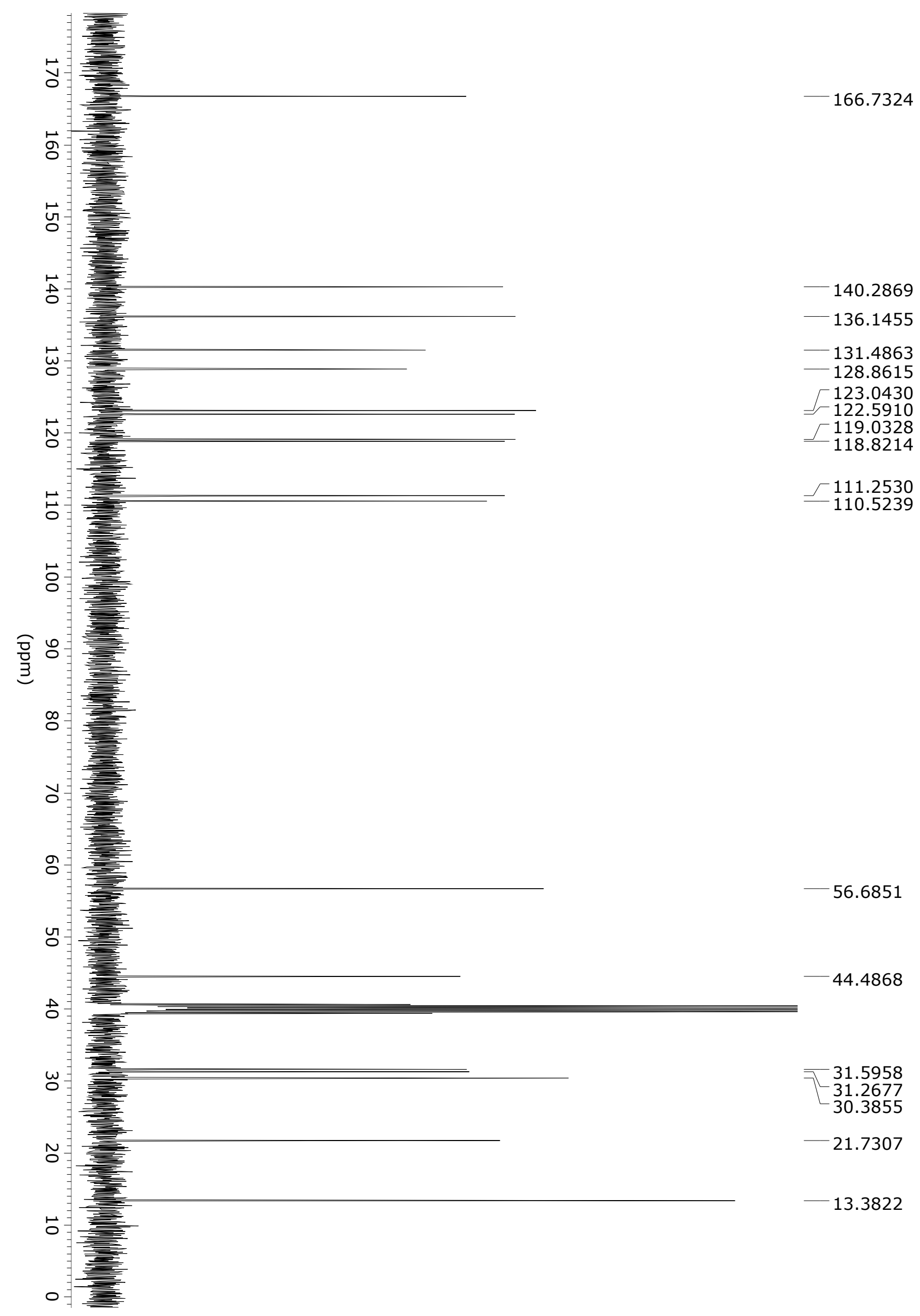




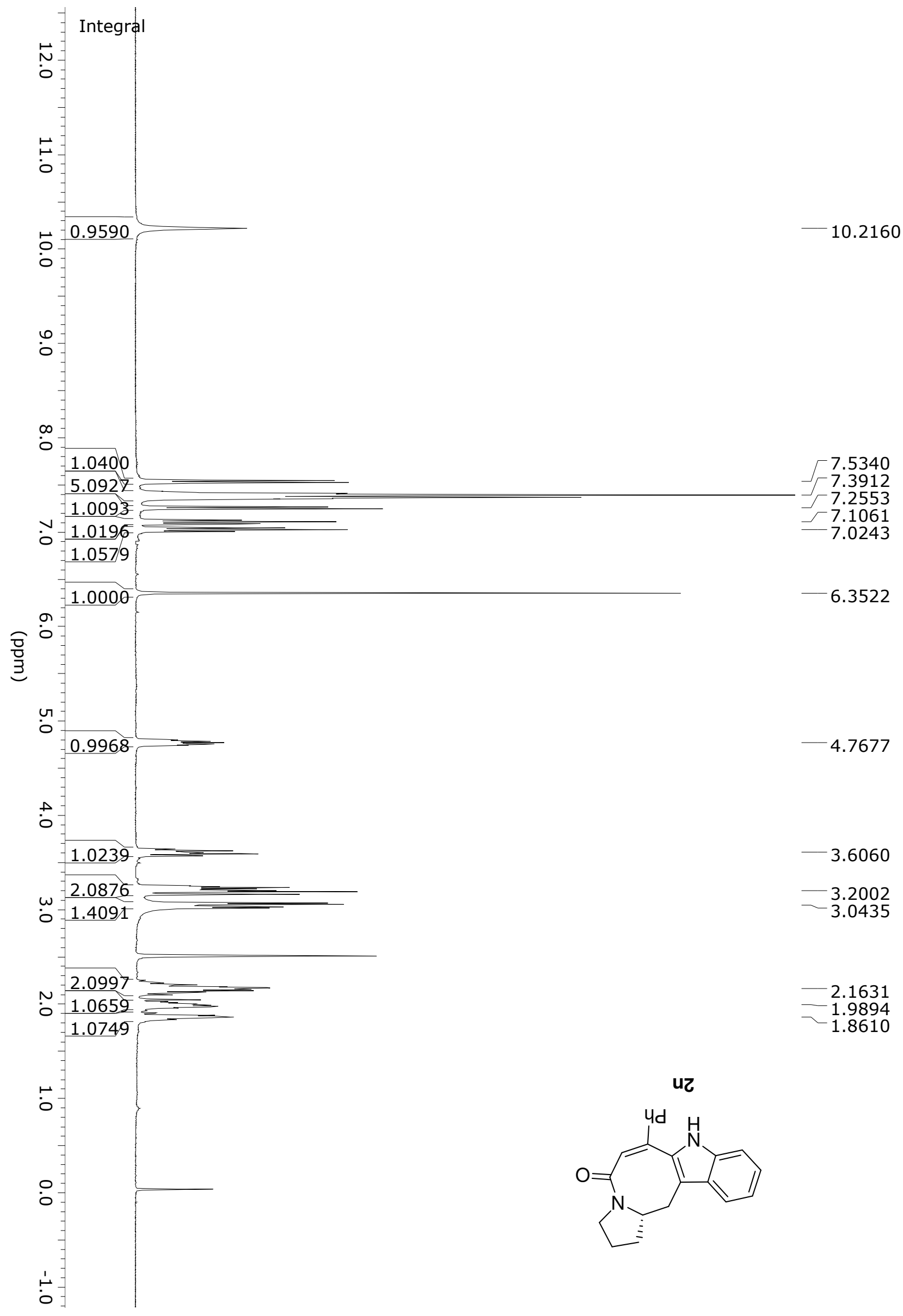




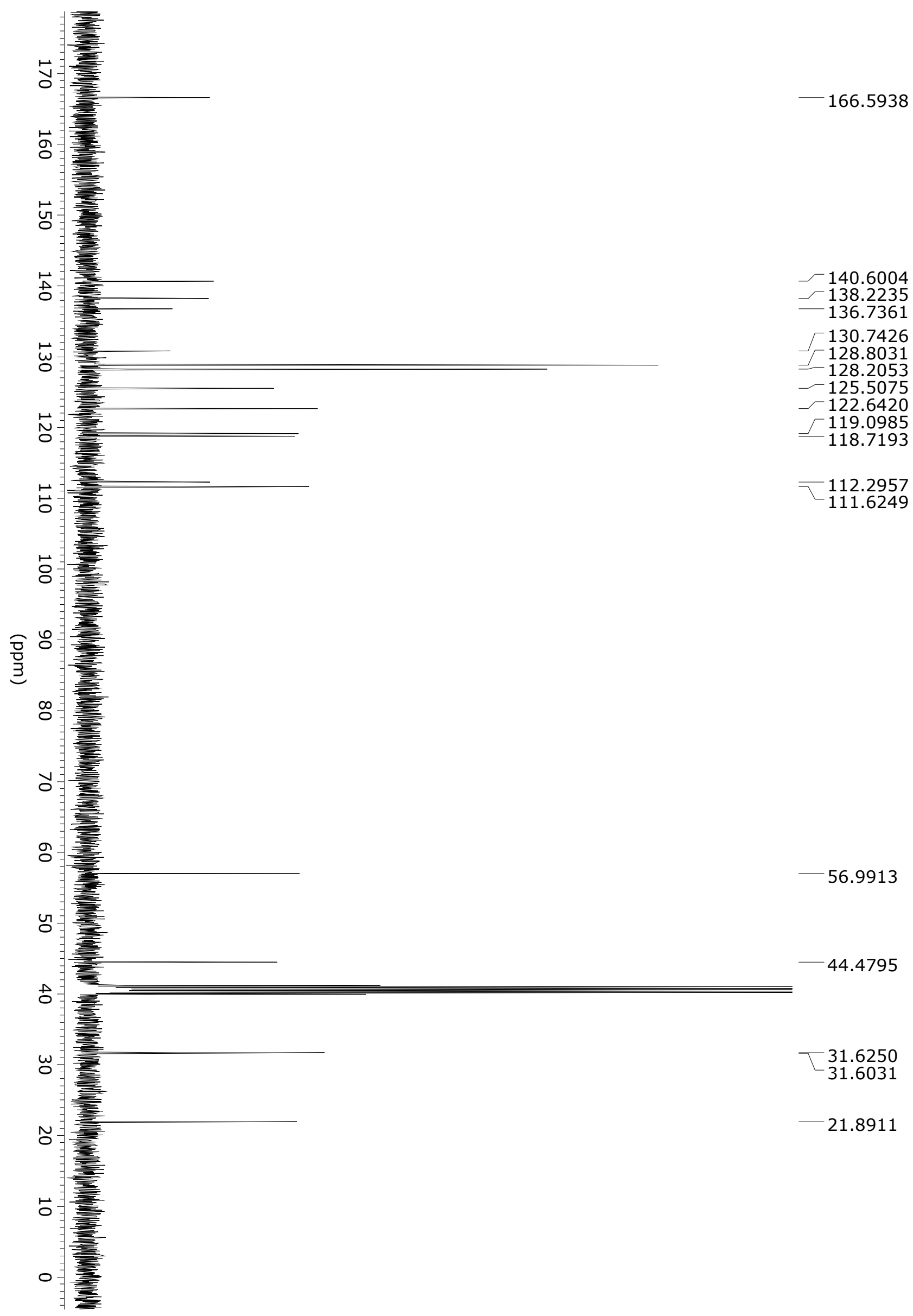




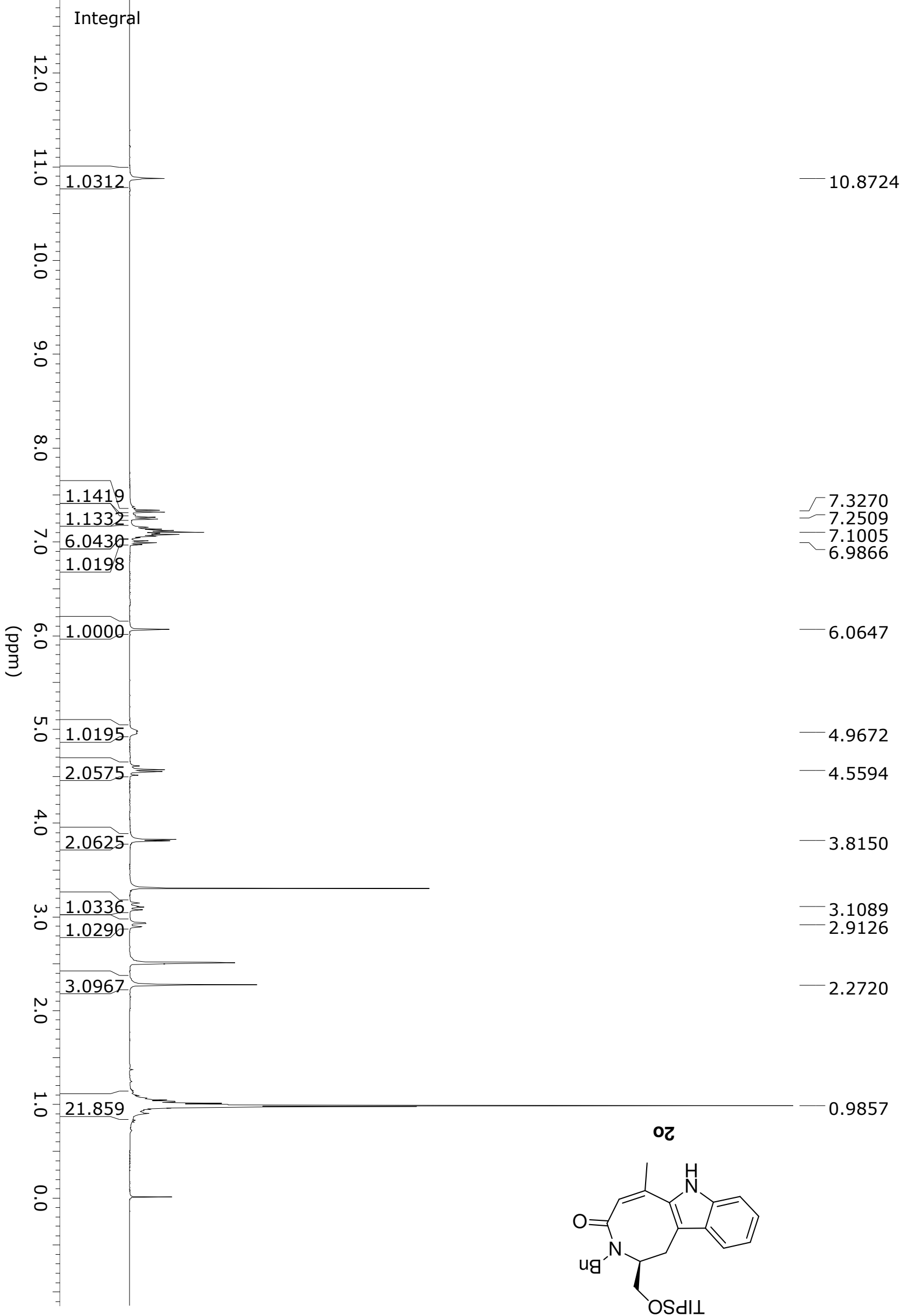




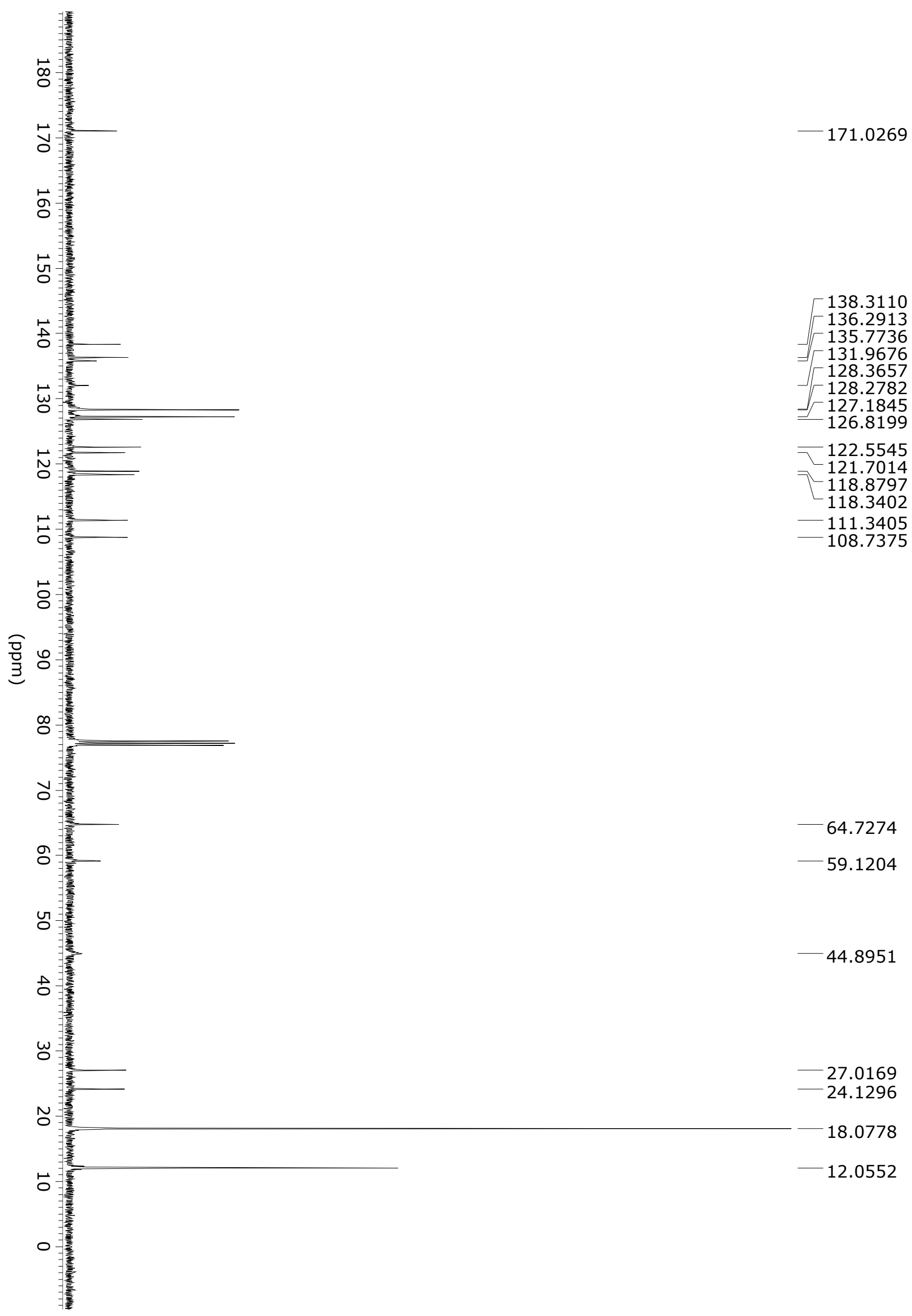




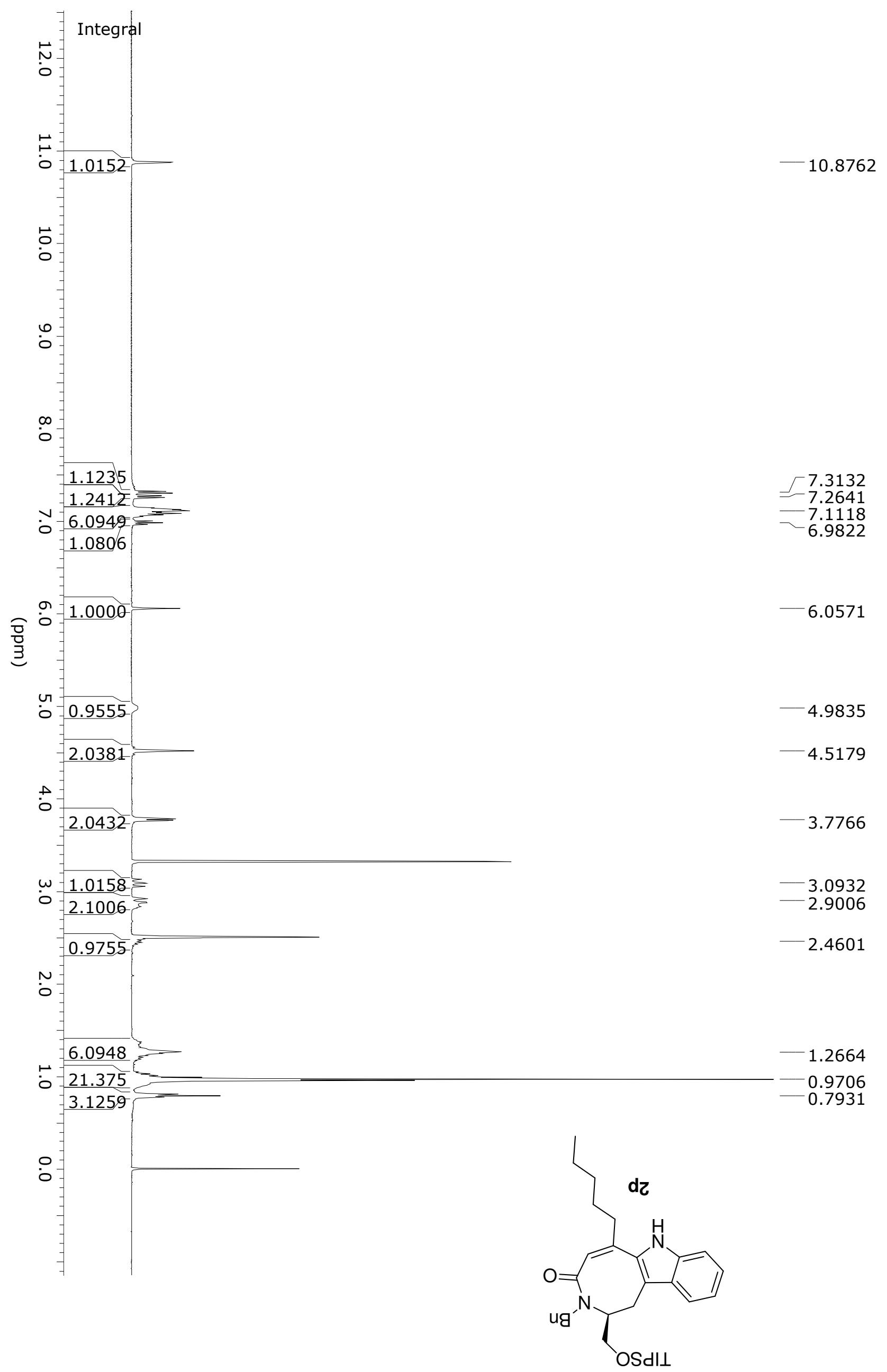




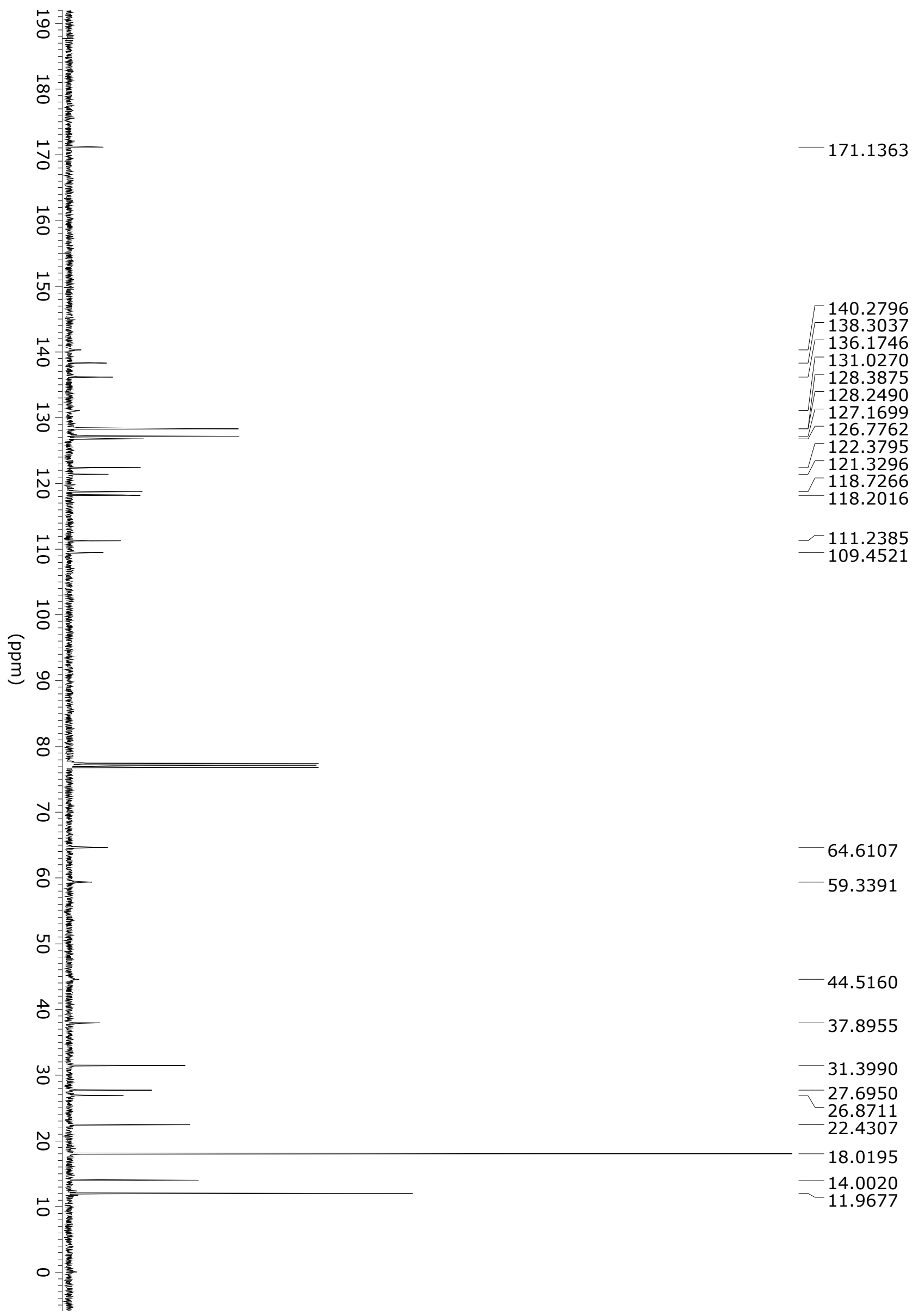




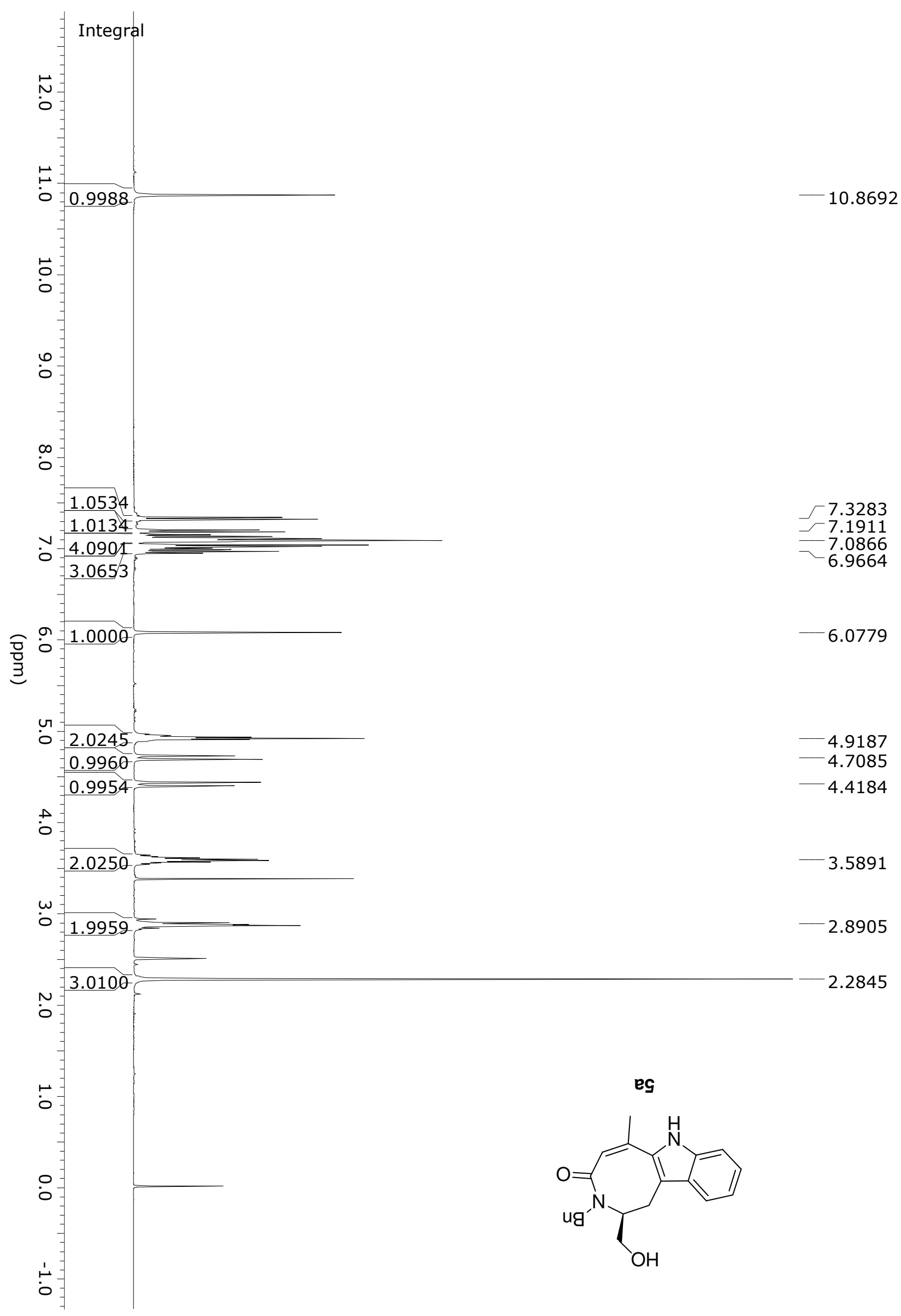




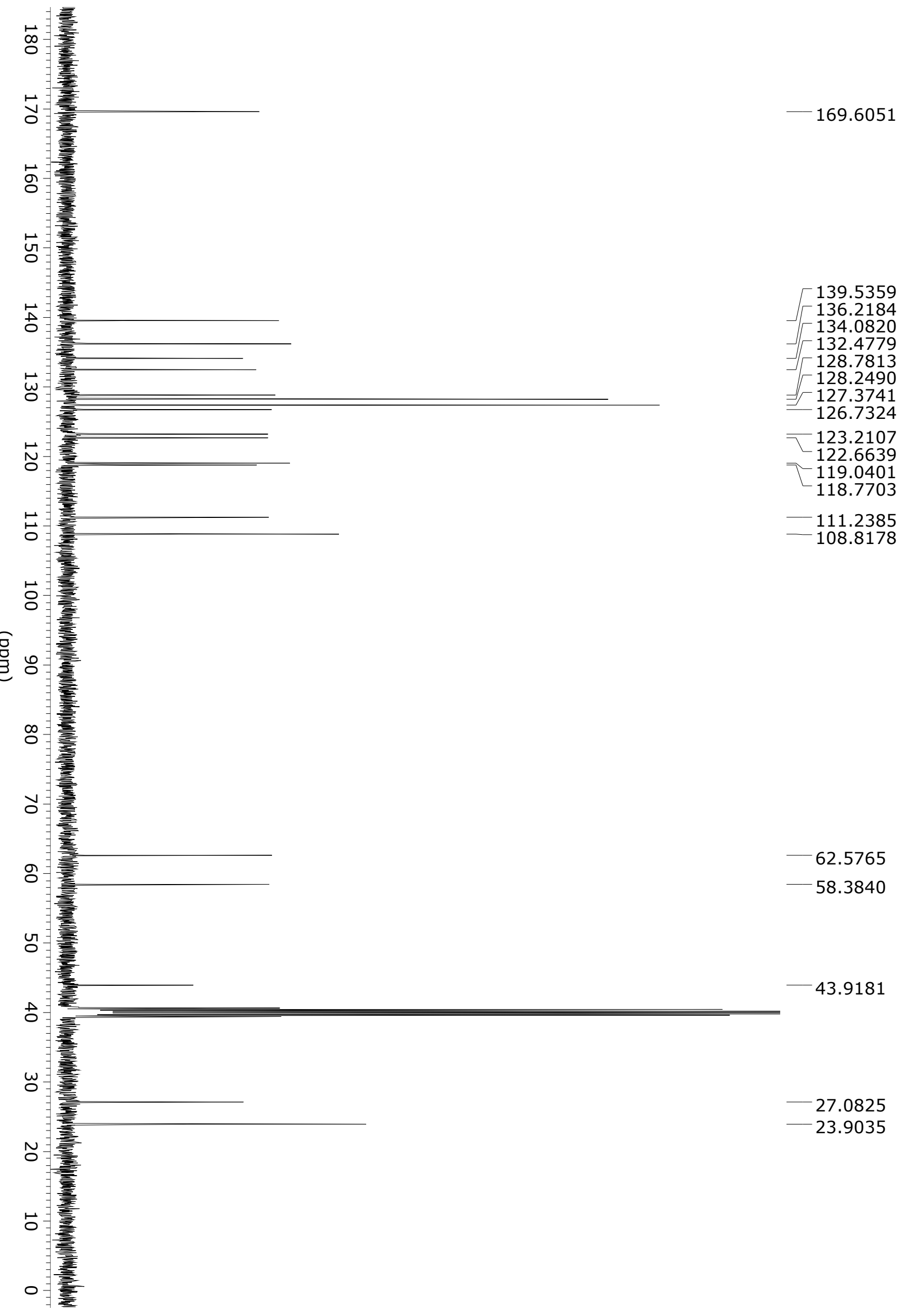




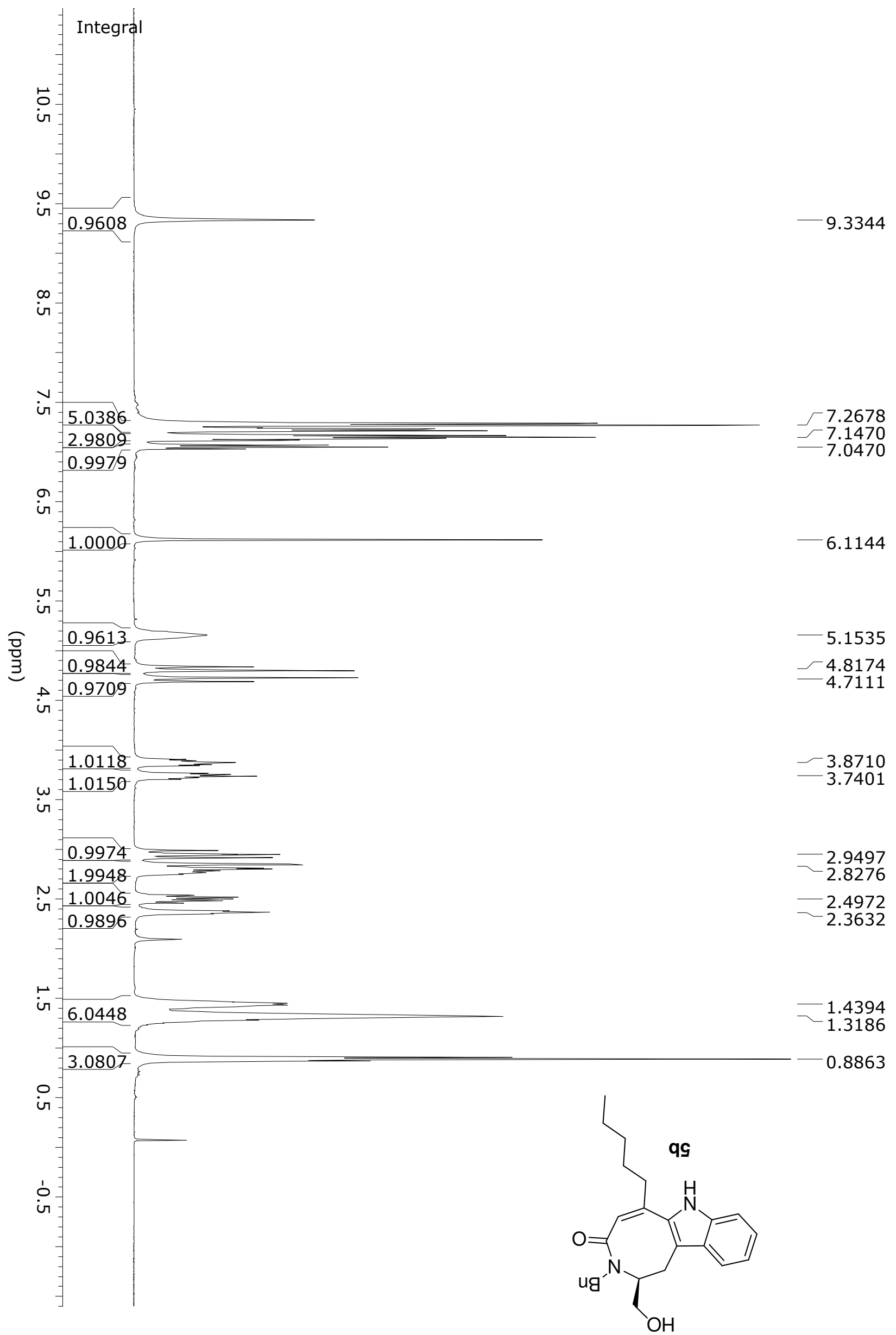




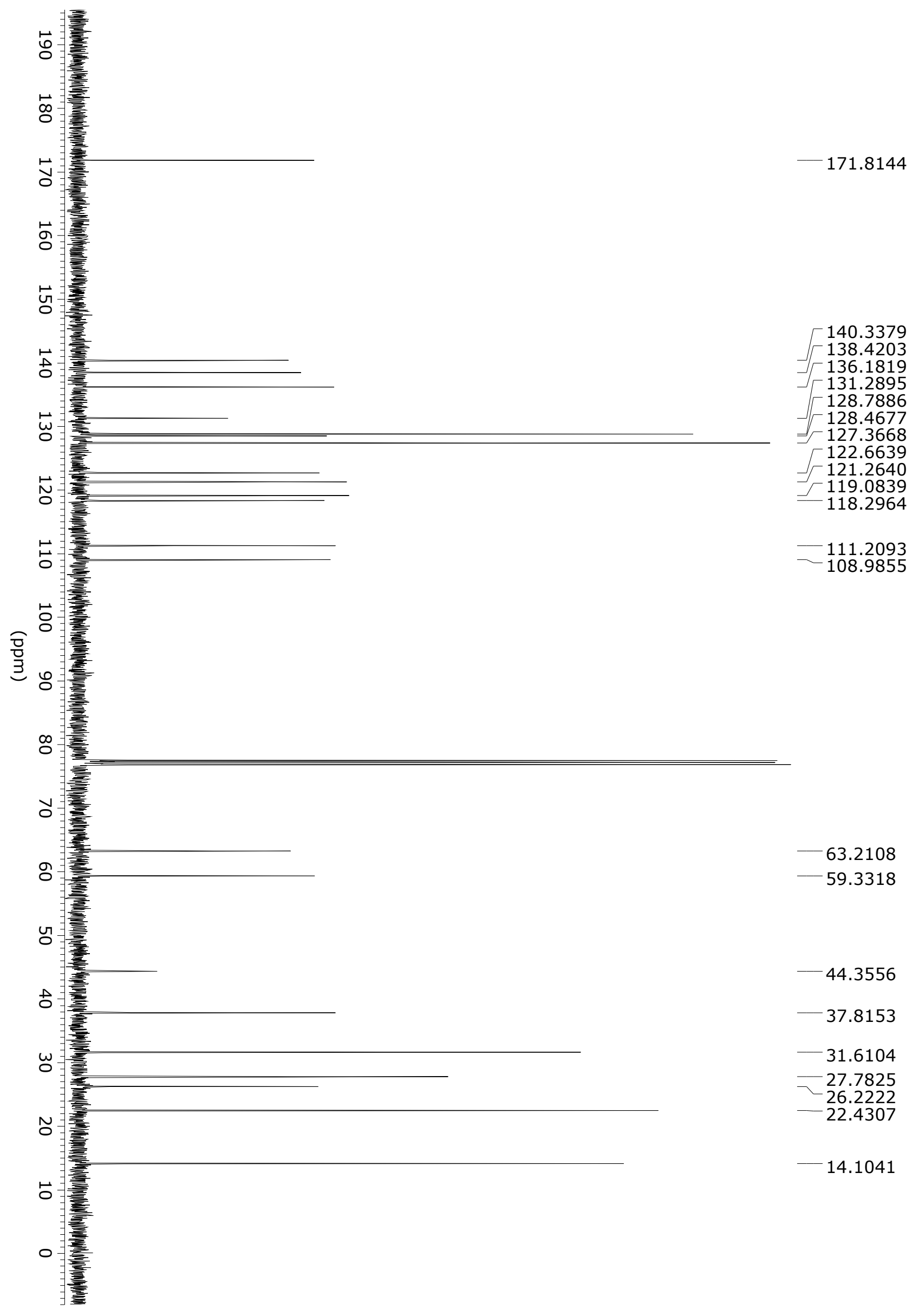




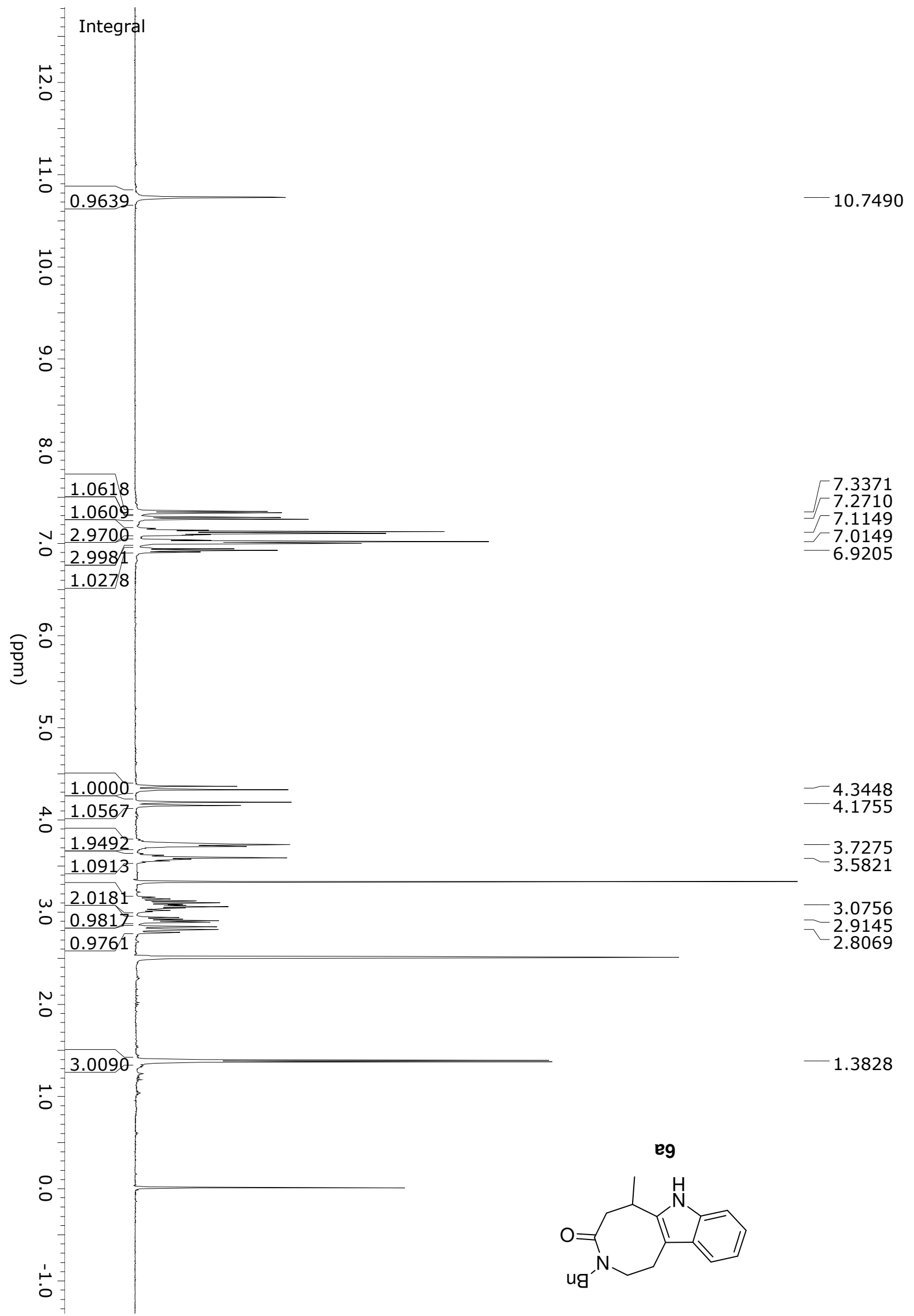




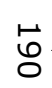

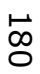

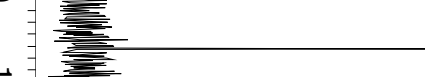

ช

官

जั

古

$-138.1433$

$-137.0350$

$=-135.2122$

129.3573

茨

$-128.3219$

政

$-127.8115$

127.1116

믐

$-121.0817$

$-118.9964$

若

$-117.1881$

官

$-110.7062$

(1)

$-105.0992$

흘

$\infty$

ช

8

ज)

$-50.1375$

$-46.2513$

b

$-41.7307$

$\omega$

$-31.9677$

No

$-22.8609$

21.6797

它

o 


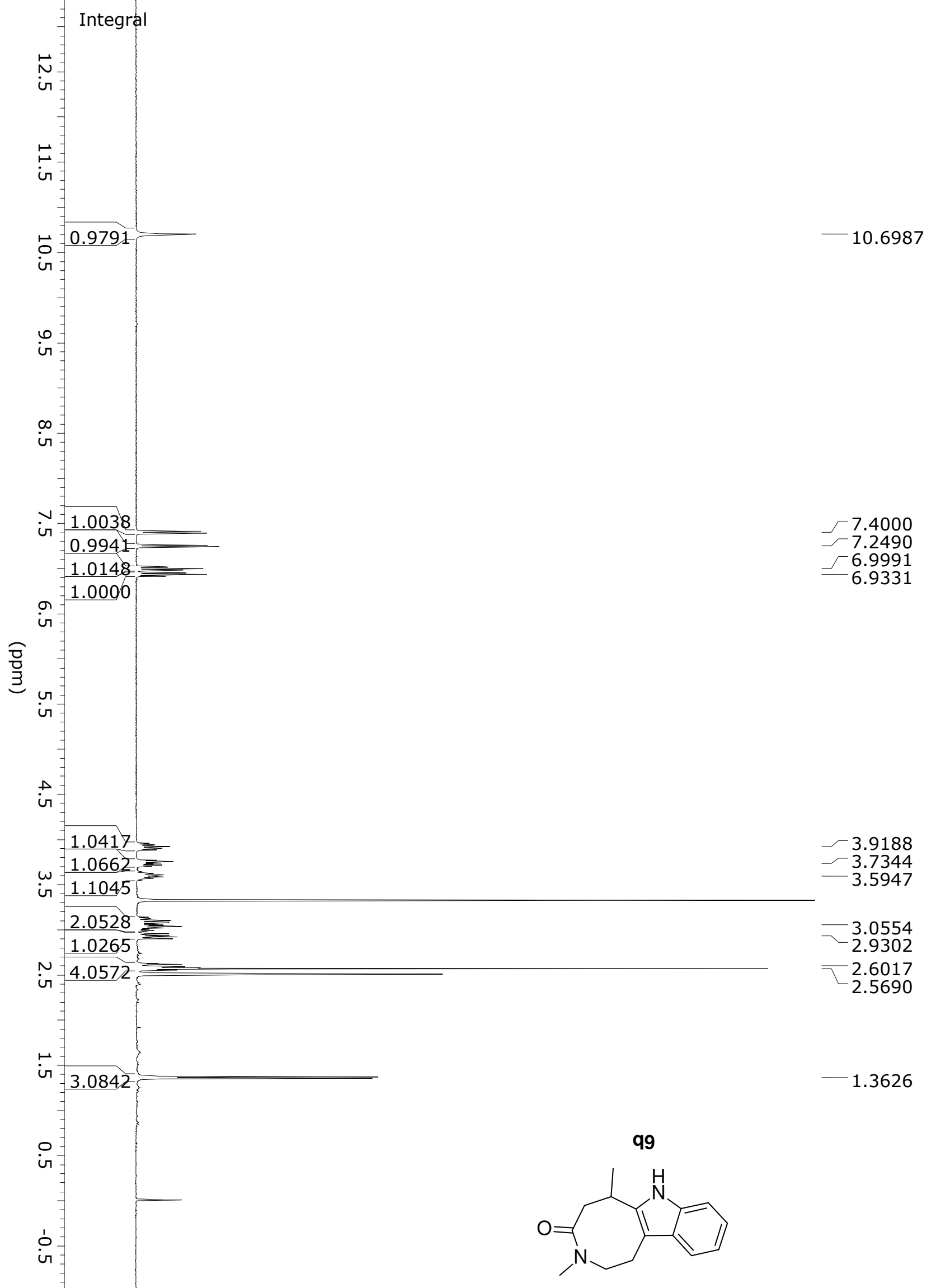




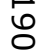

$\infty$

ป

م

ज̃

品

$-138.6755$

- 135.4090

w

$-129.6489$

N

120.6588

118.6610

$-117.4725$

苢

$-111.0635$

$-104.9607$

훙

๖

$\infty$

రㄹ.

g

ज

$-48.1762$

$-42.6786$

b

古

$\omega$

33.6520

- 30.6625

N

$-22.6567$

$-21.3224$

เ

○ 


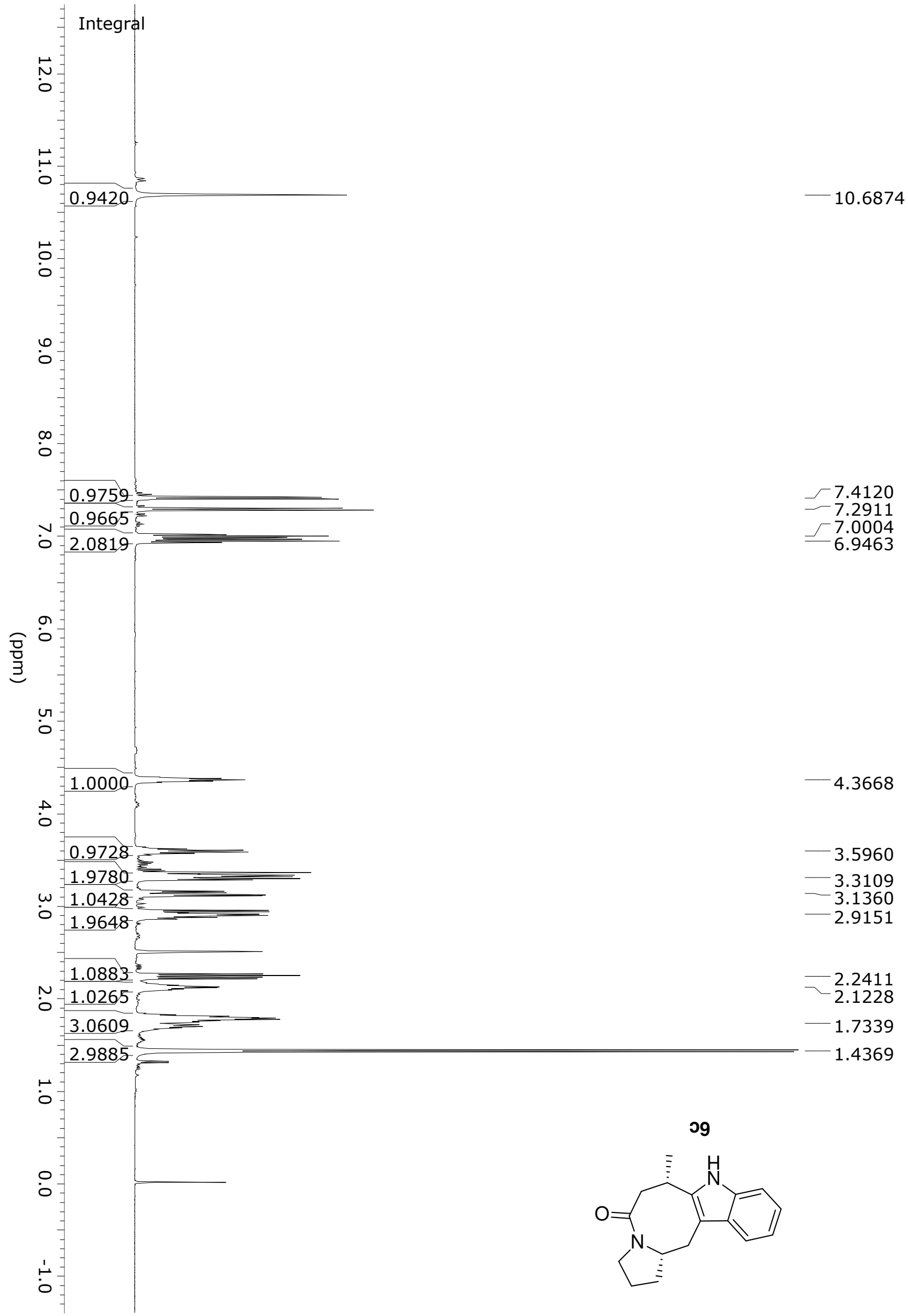




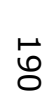

s

ํㅡㅁ

응

ज:

古

- 139.4192

$-135.3361$

$\omega$

$-129.6343$

$\stackrel{a}{\circ}$

120.4692

$-118.7995$

117.4871

点

$-111.2530$

$-105.8429$

$\frac{7}{3}$

$\infty$

ชั

8

$-57.3195$

जั

样

b

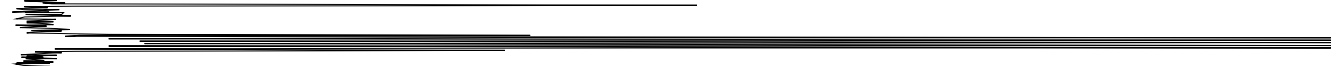

$-46.6377$

$-43.0796$

- 34.2717

$w$

29.9188

29.7001

N

$-21.6141$

$-19.4996$

它

0 


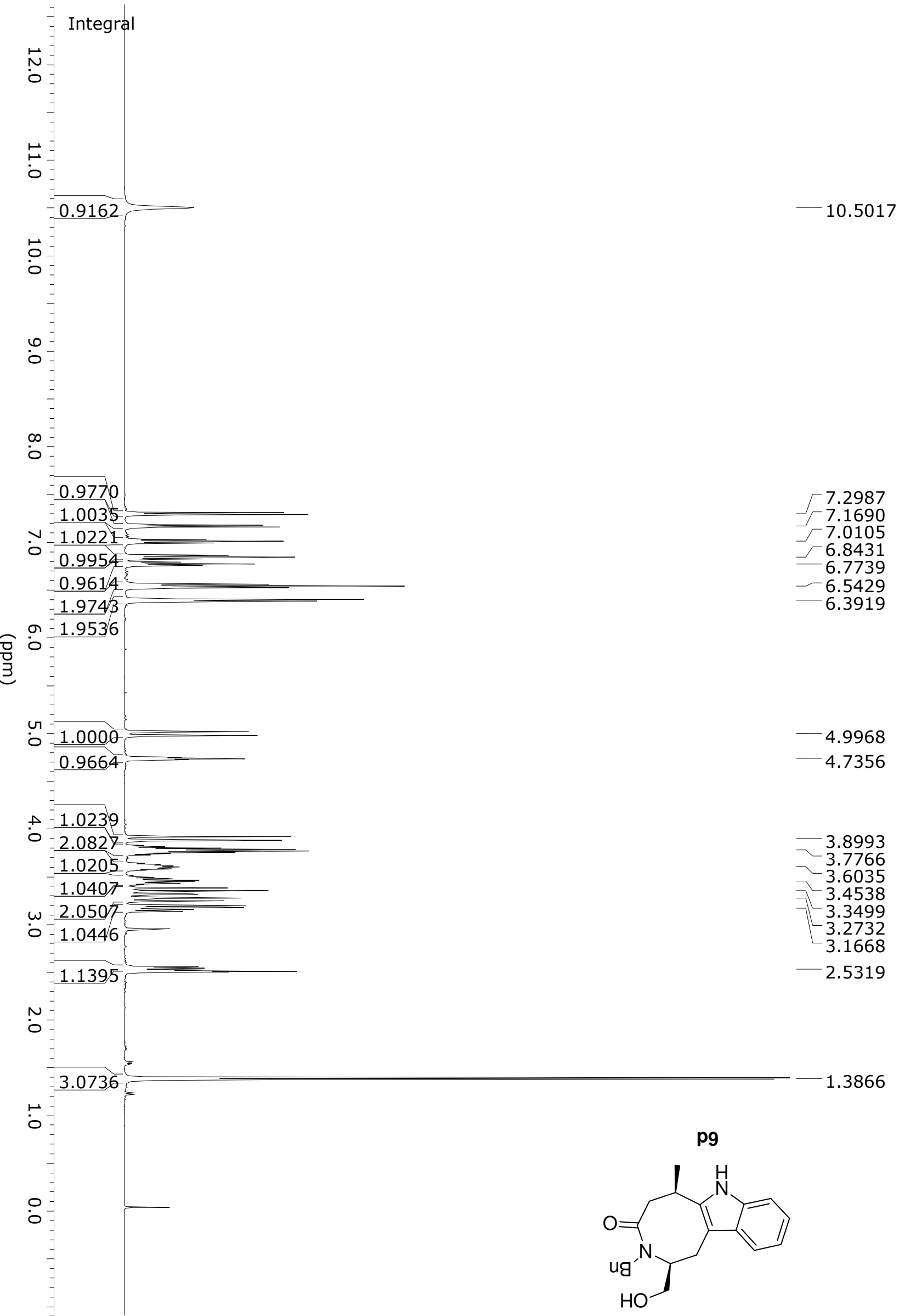


ठ

。ㅋ.

जั

品



N

吕

용․

훙

๖

$\infty$

○

。

64.4212

ज勺

$-140.2286$

$-138.1506$

$-136.0361$

129.6343

$-127.6511$

-127.0459
-126.0908

$-120.5057$

$-118.6026$

$-117.2902$

$-110.8229$

$-105.5294$

章

旁
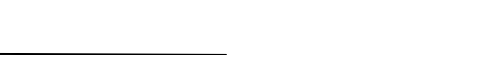


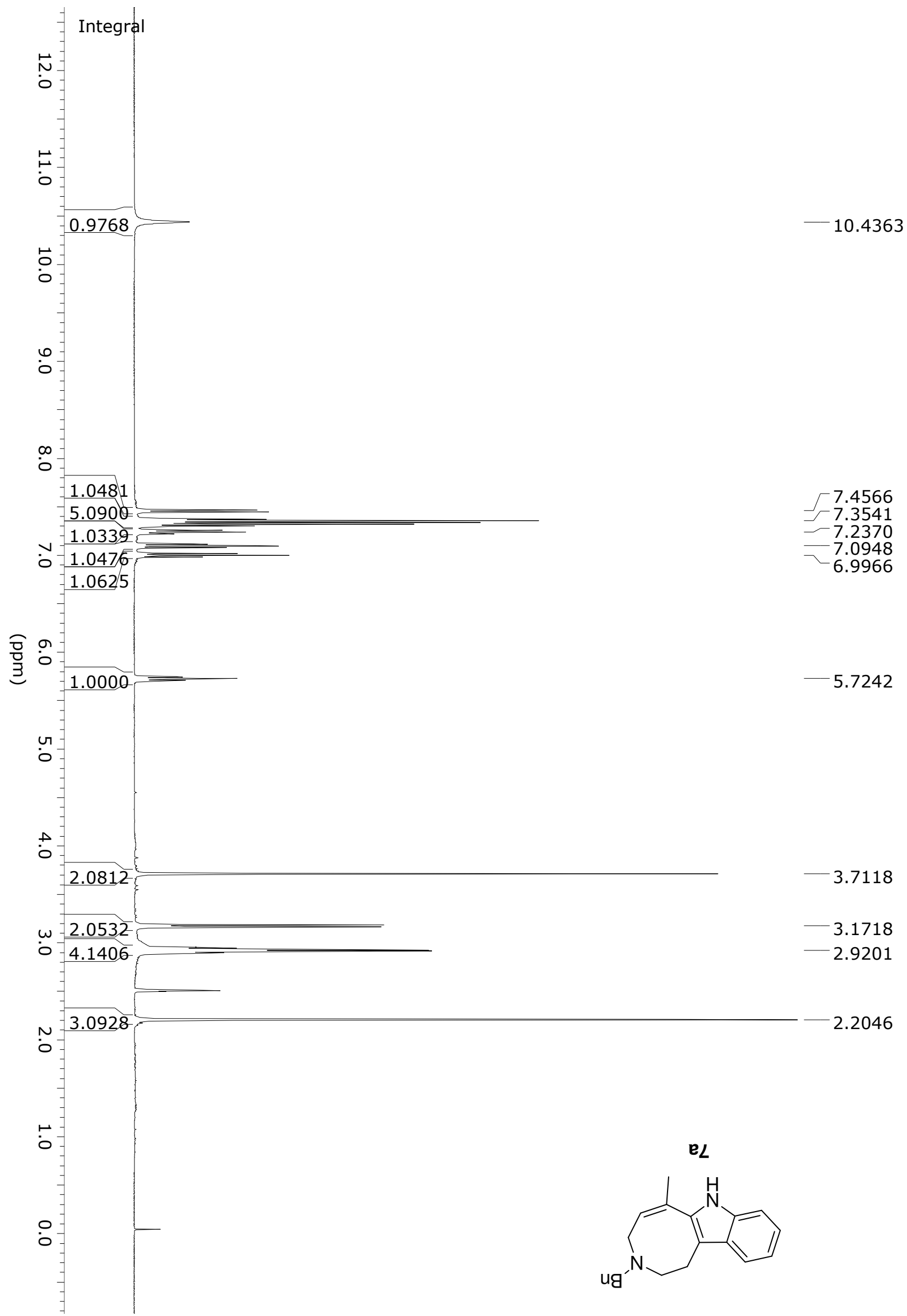




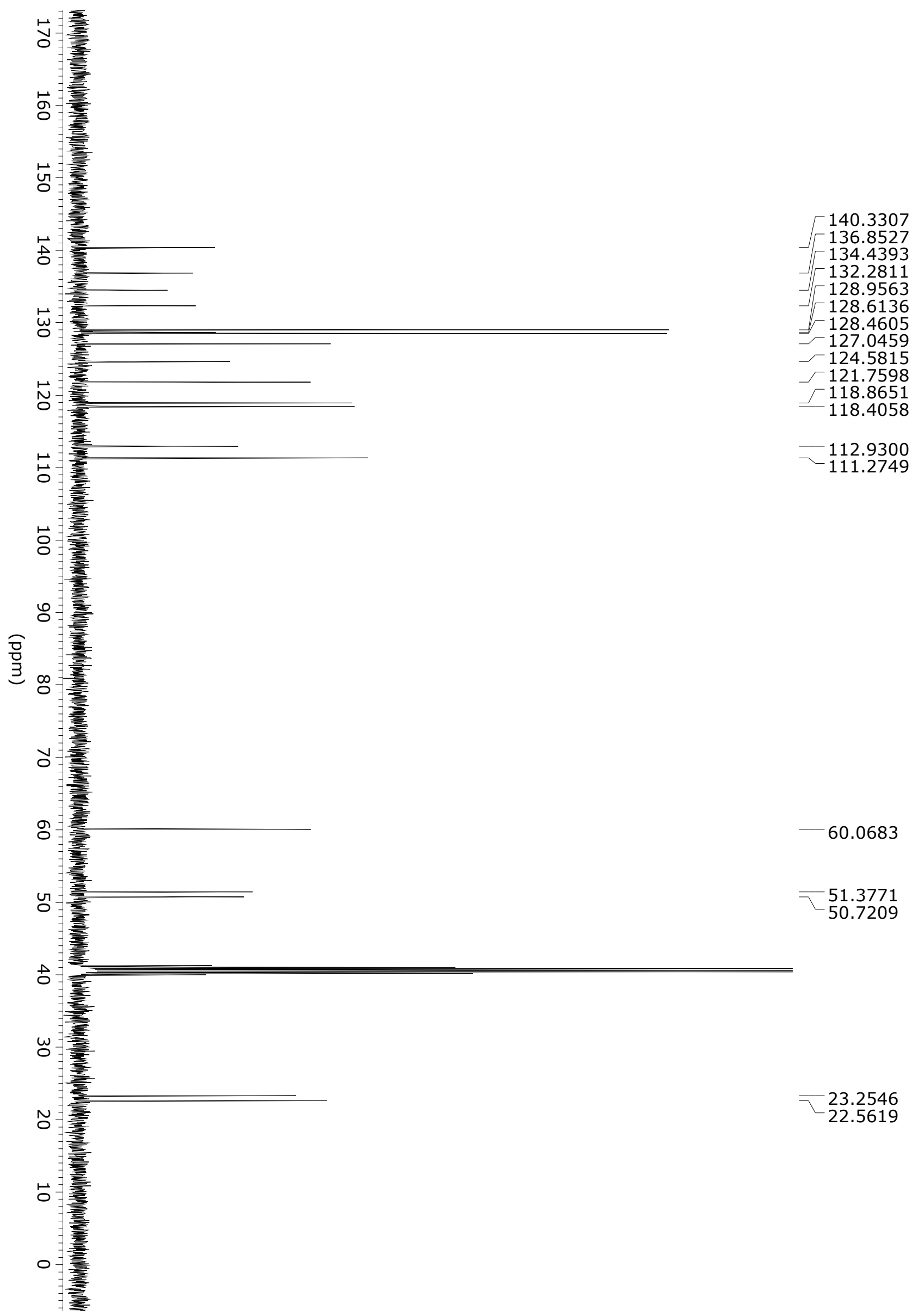




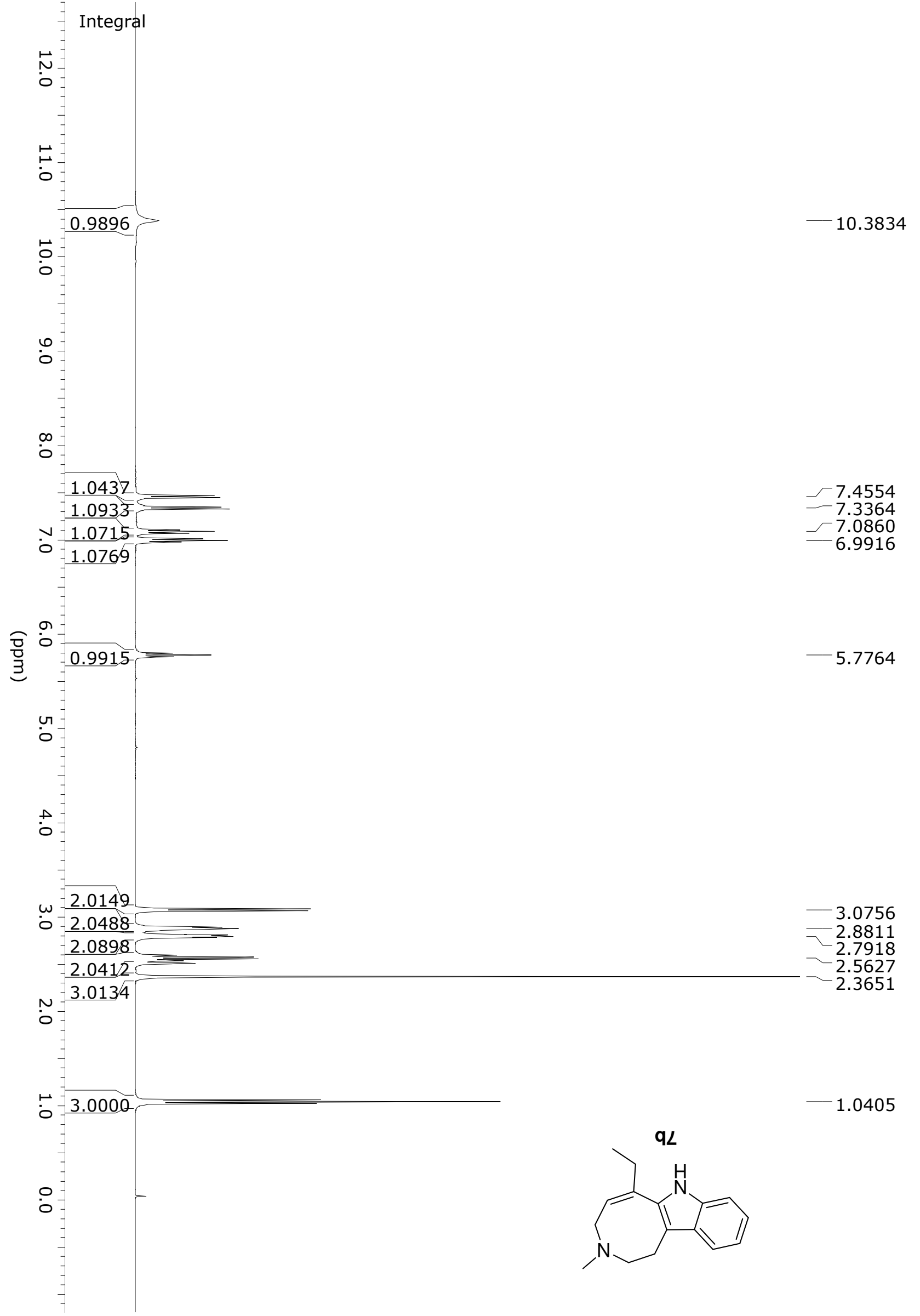




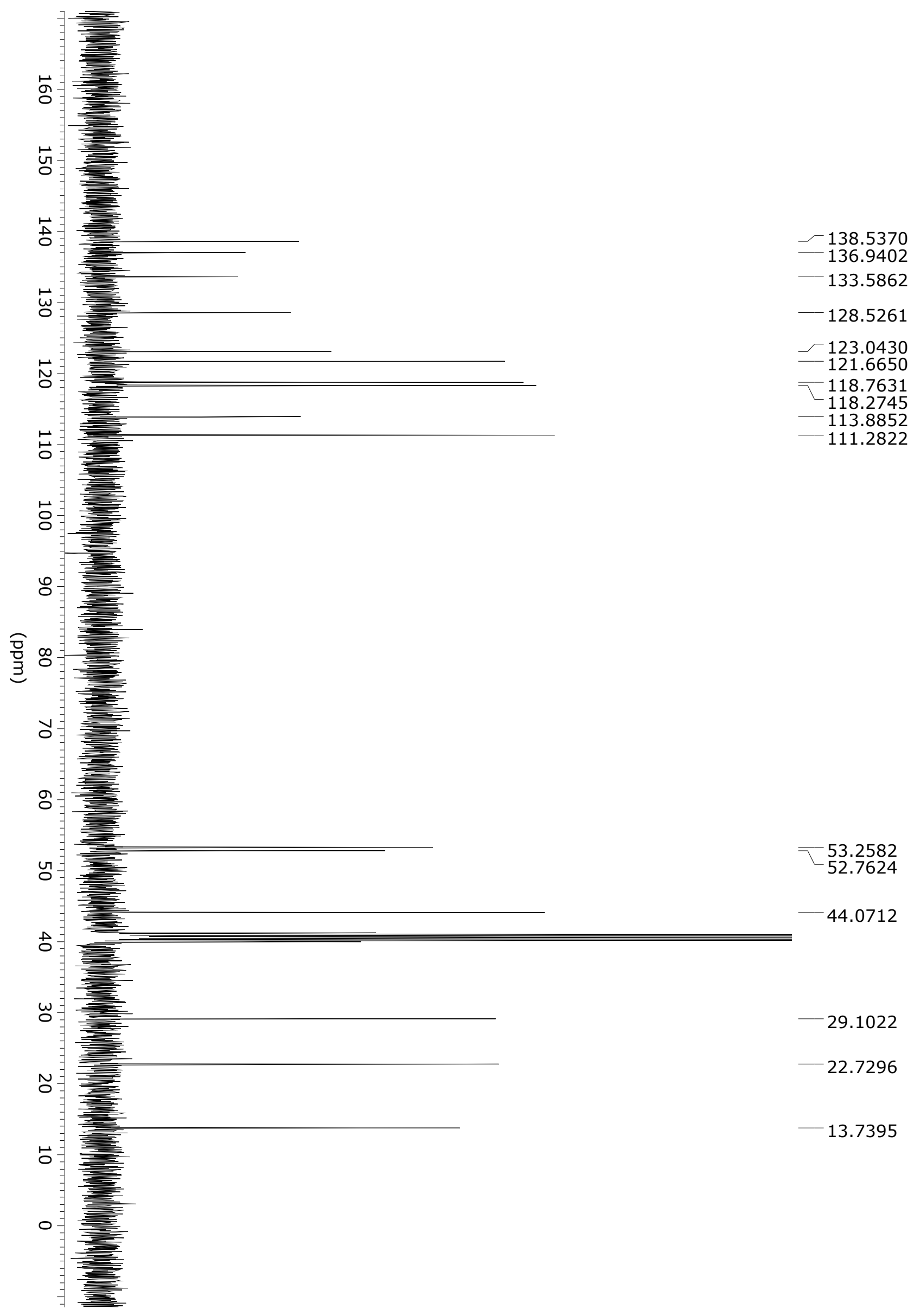




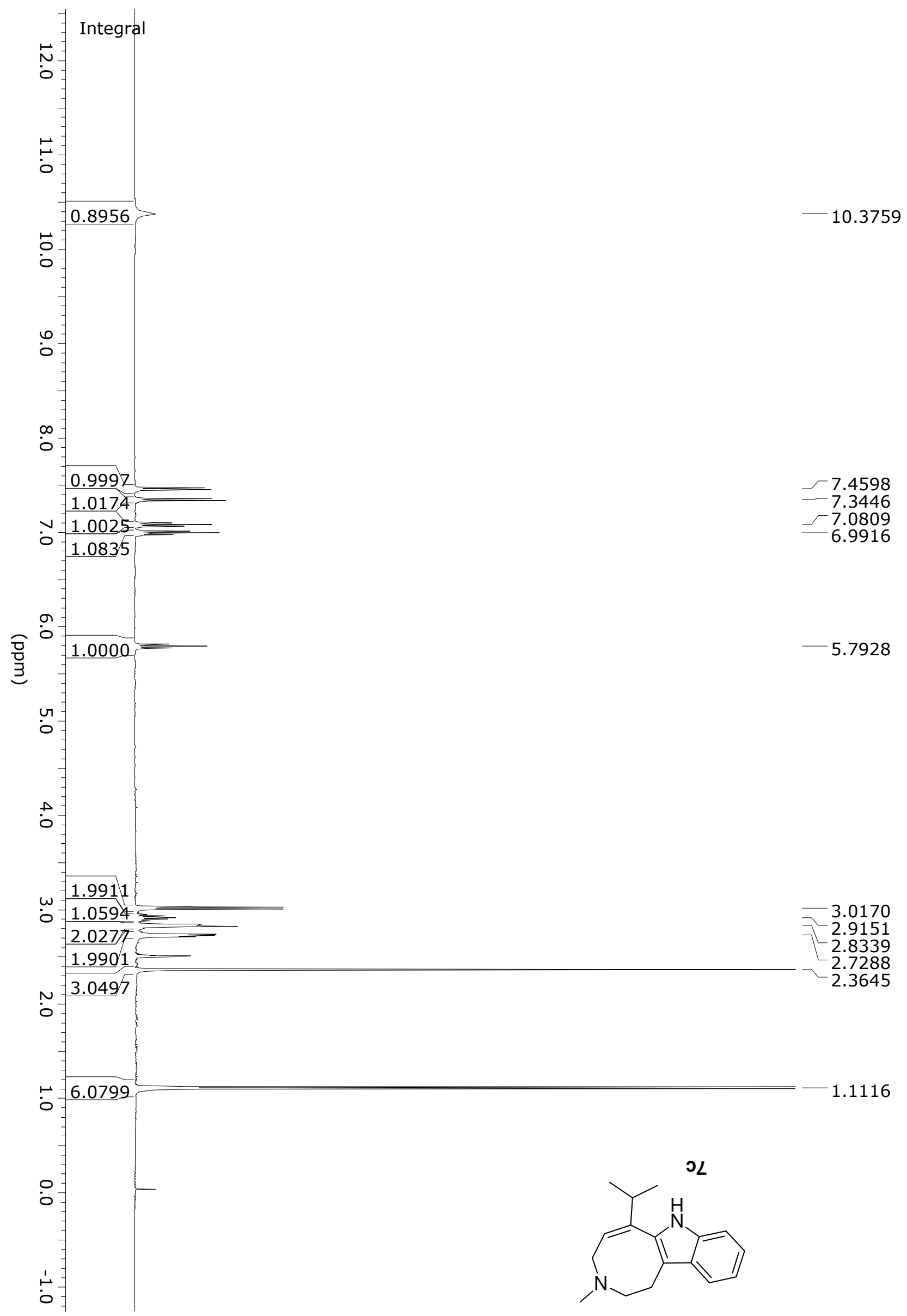




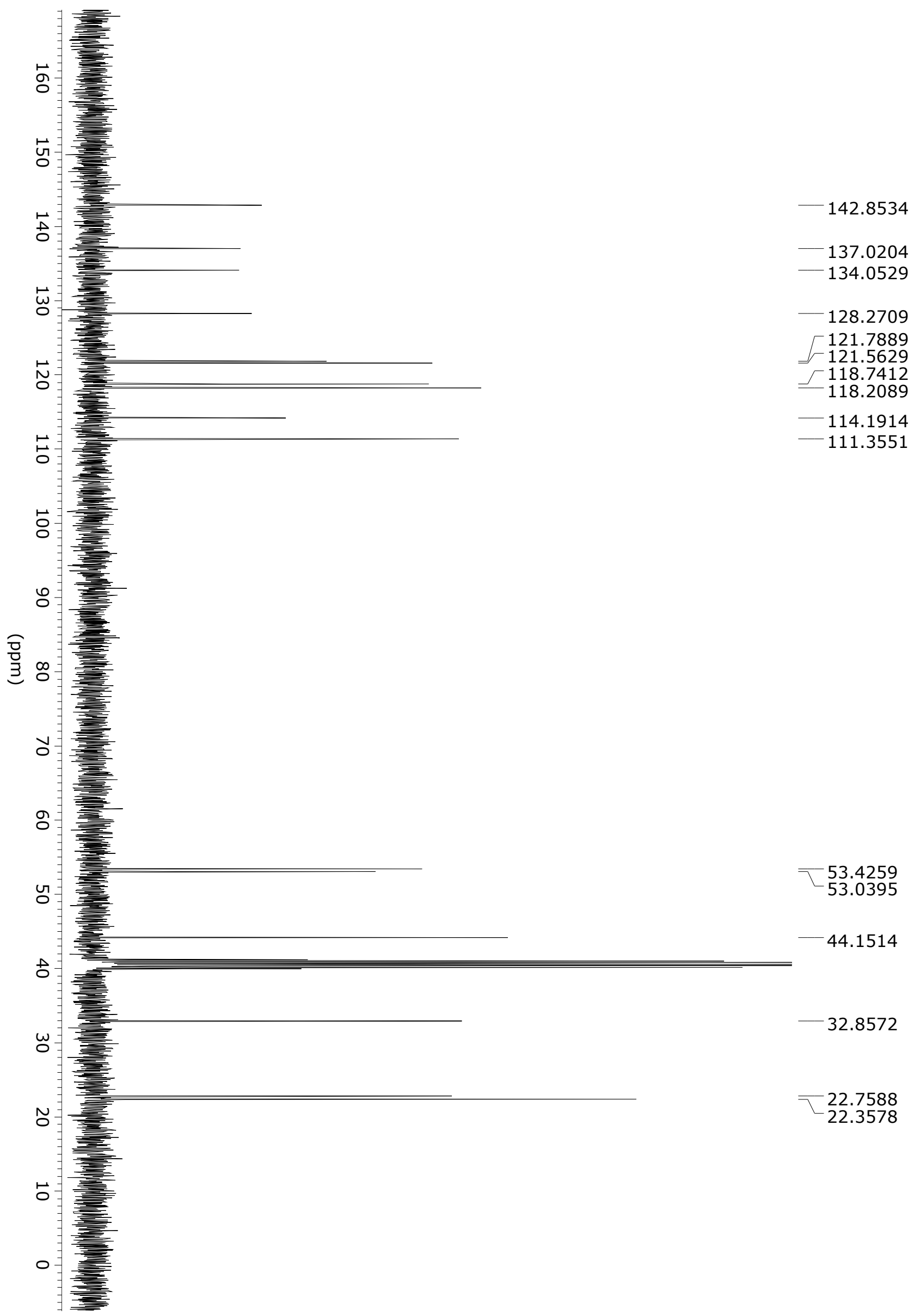

